Portland State University

PDXScholar

$3-5-2021$

\title{
Get Your Model Out There: Advancing Methods for Developing and Using Causal-Loop Diagrams
}

Erin Suzanne Kenzie

Portland State University

Follow this and additional works at: https://pdxscholar.library.pdx.edu/open_access_etds

Part of the Public Health Commons

Let us know how access to this document benefits you.

\section{Recommended Citation}

Kenzie, Erin Suzanne, "Get Your Model Out There: Advancing Methods for Developing and Using CausalLoop Diagrams" (2021). Dissertations and Theses. Paper 5664.

https://doi.org/10.15760/etd.7536

This Dissertation is brought to you for free and open access. It has been accepted for inclusion in Dissertations and Theses by an authorized administrator of PDXScholar. Please contact us if we can make this document more accessible: pdxscholar@pdx.edu. 
Get Your Model Out There:

Advancing Methods for Developing and Using Causal-Loop Diagrams

by

Erin Suzanne Kenzie

A dissertation submitted in partial fulfillment of the requirements for the degree of

\author{
Doctor of Philosophy \\ in \\ Systems Science
}

Dissertation Committee:

Wayne Wakeland, Chair

Melinda Davis

Billie Sandberg

Antonie Jetter

Portland State University

2021 
(C) 2021 Erin Suzanne Kenzie 


\begin{abstract}
As simple visual diagrams of key dynamics in complex systems, causal-loop diagrams could meet known needs in settings such as theory-based program evaluation and qualitative research. Methods for developing and using causalloop diagrams, however, are underdeveloped. This dissertation comprises three articles that advance these methods. The first paper describes a systematic review of evaluation studies utilizing causal-loop diagramming to illustrate program theory. The second paper pilots an improved method for systematically generating causal-loop diagrams from qualitative data. The third paper presents a protocol for an interview-based approach to mapping mental models. Together, this research contributes to recognizing the modeler as co-creator, reframes the relationship between intervention and context, and enables more diverse uses for causal-loop diagrams. Ultimately, this research serves to improve the rigor and transparency of methods for developing causal-loop diagrams, broadening their potential applications for modeling, research, and evaluation.
\end{abstract}




\section{Dedication}

This work is dedicated to my husband, Chris, and daughters, Magnolia and Geneva. I love you.

This dissertation is also dedicated to the memory of Sylvia Culp, who inspired my love of science, and to Donella Meadows, who brought systems thinking to life. 


\section{Acknowledgements}

I owe an enormous debt of gratitude to Wayne Wakeland for being a (truly) tireless mentor and advocate. Wayne, the energy you bring to your work and your dedication to students is unmatched. I am also grateful for the support of my other dissertation committee members-Melinda Davis, Billie Sandberg, and Antonie Jetter-for seeing me through this process during an impossible year.

A big thanks to Gina Villarreal and Henner Busch for all of the support and encouragement over the years and to the facilitators I interviewed for sharing their perspectives.

I would also like to acknowledge the PEO organization for their generous PEO Scholar Award, which allowed me to complete my dissertation research.

And finally, this work would not have been possible without the unwavering support of my husband, Chris Allen. Thank you for wrangling our kids during a pandemic so I could write about diagrams. 
Table of Contents

Abstract

Dedication

Acknowledgements

List of Tables

List of Figures

List of Abbreviations

1. Introduction

1.1. Motivation

1.1.1. Origins and uses for causal-loop diagramming

1.1.2. Current gaps in knowledge

1.2. Research questions, methods, and papers

1.3. Significance

1.4. Causal-loop diagram notation

1.5. Terminology

2. Paper \#1: Mapping complexity-aware program theory with causal-loop diagramming: A systematic review of mixed-method evaluation studies

2.1. Abstract

2.2. Introduction

2.2.1. Standard logic models and diagrams of program theory

2.2.2. Causal-loop diagramming

2.3. Methods

2.4. Results

2.4.1. Reasons for using causal-loop diagramming

2.4.2. Diagram development

2.4.3. Diagram features

2.4.4. Diagram analysis and use

32

2.4.5. Identified strengths and limitations of causal-loop diagramming for program theory

2.4.6. Centering the intervention vs. the problem

2.5. Discussion

2.5.1. Summary of findings

40

2.5.2. Comparing methods from included studies to system dynamics best practice

40

2.5.3. Aligning diagram development with system dynamics best practices

2.5.4. Limitations

2.5.5. Future research

48

2.6. Conclusion

49

50 
3. Paper \#2: Reclaiming the 'loop' in causal-loop diagram: Advancing methods for identifying causal structures in qualitative data 51

3.1. Abstract 51

3.2. Introduction 51

3.3. Study setting 57

3.4. Recognizing causal structures 58

3.5. Analysis process 64

3.5.1. Coding for causal structures (step 3) 66

3.5.2. Generation of query reports and sketching causal structures (steps 4 and 5) 71

3.5.3. Creation of causal mapping tables (step 6) 72

3.5.4. Generation of causal-loop diagram from causal mapping table (steps 7 and 8) 73

3.5.5. Use of causal-loop diagrams to understand mental models (step 9)

3.6. Results

3.7. Discussion 81

3.7.1. Summary of findings 81

3.7.2. Advantages and limitations of approach 82

3.7.3. System dynamics applications $\quad 86$

$\begin{array}{ll}\text { 3.7.4. Qualitative applications } & 87\end{array}$

3.7.5. Ramifications for automated model generation 87

3.7.6. Future research 88

3.8. Conclusion $\quad 89$

4. Paper \#3: Advancing interview-based methods for mapping mental models using causal-loop diagramming $\quad 90$

$\begin{array}{ll}\text { 4.1. Abstract } & 90\end{array}$

4.2. Introduction 90

4.3. Interviewing approaches 94

4.3.1. Qualitative interviewing 94

4.3.2. Interviewing in system dynamics $\quad 95$

4.3.3. Realist interviewing 97

4.4. Overview of interview-based process for diagramming mental models 99

4.4.1. Boundary definition and planning 102

4.4.2. Initial interviews $\quad 105$

4.4.3 Mapping analysis $\quad 114$

4.4.4 Follow-up interviews $\quad 115$

4.4.5. Participatory model review 116

4.5. Potential applications $\quad 117$

4.5.1. System dynamics modeling 117

4.5.2. Program evaluation 118

4.5.3. Qualitative research 118 
4.6. Discussion

4.6.1. Experimental comparison with group model building

4.6.2. Choosing between interview-based modeling and other methods 120

4.6.3. Future research

121

4.7. Conclusion

121

5. Synthesis

123

5.1. Contributions to knowledge

123

5.1.1. Capturing implied information in mapping mental models

123

5.1.2. Modeler as co-creator

124

5.1.3. Causal-loop diagrams as a problem-centric approach

124

5.1.4. Causal-loop diagrams as representations of knowledge

127

5.2. Implications / Significance

128

5.3. Limitations

129

5.4. Future research

131

5.5. Conclusion

132

5.6. Postscript

133

References

136

Appendices

149

Appendix A: Examples of Theory of Change diagrams 149

Appendix B: Literature Review Protocol

151

Appendix C: Sample of causal-loop diagrams from studies included in review

152

Appendix D: Casual mapping table sample

158

Appendix E: Selection of diagrams produced during initial interviews 164 


\section{List of Tables}

Table 1. Evaluation studies utilizing causal-loop diagrams for program theory

Table 2. Strengths of causal-loop diagramming for program theory identified in included studies.

Table 3. Limitations of causal-loop diagramming for program theory identified in included studies.

Table 4. Analysis process for generating causal map from qualitative data

64

Table 5. Codes indicating model components used during analysis. $\quad 67$

Table 6. Coding examples from ORPRN study. 68

Table 7. Design features of causal-loop diagrams 70

Table 8. Prompts for analyzing causal-loop diagrams generated from qualitative data

Table 9. Characteristics of causal-loop diagrams gleaned from analysis of practice facilitator interviews.

Table 10. Data associated with steps in diagramming a feedback loop.

Table 11. Proposed interview-based protocol for mapping individual mental models

100

Table 12. Components of causal-loop diagrams 104

Table 13. Design criteria for initial interview

105

Table 14. Comparison of features of modeler-led system dynamics, group model building, interview-based model building, and standard semistructured qualitative interviews, with positive characteristics highlighted in green 


\section{List of Figures}

Figure 1. Dissertation papers mapped onto components of interview-based approach to mapping mental models with causal-loop diagrams.

Figure 2. Three dissertation papers situated within fields of inquiry.

Figure 3. Example of causal-loop diagram used for complexity-aware program theory.

Figure 4. Generic structures of reinforcing and feedback loops with corresponding graphs of behavior over time.

Figure 5. Basic format of a "pipeline" logic model. Reproduced from W.K. Kellogg Foundation 2004.

Figure 6. Comparison of diagram connection types.

Figure 7. Process of article selection for review.

Figure 8. Example causal-loop diagram from an included study showing "growth and underinvestment" archetype.

Figure 9. Iterative modeling process embedded in context.

Figure 10. Hierarchical relationships between variables, causal links, feedback loops, and archetypes.

Figure 11. Types of system boundaries for causal-loop diagramming.

Figure 12. Generic structure and example of goal-directed balancing feedback loops.

Figure 13. Causal structures surrounding clinician and staff motivation and buy-in (C/S motivation \& buy-in) across four participants.

Figure 14. Goal-directed balancing feedback loops describing practice SBIRT quality improvement process.

Figure 15. Modeler hypothesis diagram showing carrying capacity archetype applied to ORPRN case.

Figure 16. Iterative, participatory process for diagramming mental models. Numbered steps correspond to numbers in Table 11.

Figure 17. Generic structures and examples of reinforcing and balancing feedback loops

Figure 18. Three common systems archetypes: shifting the burden (Figure $18 \mathrm{~A})$, success to the successful (Figure 18B), and escalation (Figure 18C). Adapted from Kim 1994.

Figure 19. Intervention-centric and problem-centric approaches to understanding systems change.

Figure 20. Dissertation papers mapped onto types of knowledge and evidence synthesized to causal-loop diagrams for program development and implementation science. 
List of Abbreviations

$\begin{array}{ll}\text { B } & \text { Balancing feedback loop } \\ \text { BI } & \text { Brief intervention } \\ \text { C/S } & \text { Clinician and staff } \\ \text { MAT } & \text { Medication-assisted treatment } \\ \text { ORPRN } & \text { Oregon Rural Practice-based Research Network } \\ \text { PERC } & \begin{array}{l}\text { Practice enhancement research coordinator, a practice facilitator } \\ \text { role at ORPRN }\end{array} \\ \text { QI } & \text { Quality improvement } \\ \text { R } & \text { Reinforcing feedback loop } \\ \text { SBIRT } & \text { Screening, brief intervention, and referral to treatment } \\ \text { UAU } & \text { Unhealthy alcohol use }\end{array}$


Remember, always, that everything you know, and everything everyone knows, is only a model. Get your model out there where it can be shot at. Invite others to challenge your assumptions and add their own. Instead of becoming a champion for one possible explanation or hypothesis or model, collect as many as possible. Consider all of them plausible until you find some evidence that causes you to rule one out. That way you will be emotionally able to see the evidence that rules out an assumption with which you might have confused your own identity.

- Donella Meadows, 2008 
1. Introduction

\subsection{Motivation}

1.1.1. Origins and uses for causal-loop diagramming

Causal-loop diagramming is a method from systems science for visually depicting causal relationships between variables in a complex system (Sterman 2000; Anderson and Johnson 1997; Richardson 1986; Lane 2008). The method was created as a way to describe feedback relationships when developing computational system dynamics models, but has since become a standalone approach. Feedback loops—a key feature of causal-loop diagrams-are the source of nonlinear behavior in systems, and are important for understanding how systems behave.

Causal-loop diagrams have been used in a variety of fields, such as business, ecology, and biomedicine (Ford 2010; Wittenborn et al. 2016; Bala, Arshad, and Noh 2017; Kenzie et al. 2018). The practitioner-oriented field of systems thinking utilizes causal-loop diagrams as part of a 'systems approach' to visualizing mental models of complex systems (Senge 2010; Stroh 2015; Zurcher, Jensen, and Mansfield 2018). They are also used as educational tools for teaching nonlinear systems (Wheat 2007; Aubrecht et al. 2019).

Several high-profile system dynamicists have criticized causal-loop diagrams for lacking the ability to generate estimated graphs of behavior over time-a key 
feature in simulation models (Richardson 1986; Forrester 2007). This critique has contributed to a preference for computational system dynamics over standalone qualitative tools in mainstream system dynamics. Simulation, however, is not always feasible or appropriate, particularly for complex sociotechnical or socialecological systems in which human behavior plays a central role (Coyle 2004). Moreover, the visual format of causal-loop diagrams may serve purposes different from estimating system behavior. Two such uses for causal-loop diagrams include conceptual models—such as diagrams of program theory for evaluation-and depiction of individuals' or groups' mental models.

\subsubsection{Current gaps in knowledge}

The field of program evaluation encompasses theories and methods for assessing the effectiveness of programs and policies (Newcomer et al. 2015). In theory-based or theory-driven evaluation, evaluators utilize explicitly articulated program theories describing how an intervention is thought to result in observed outcomes (Weiss 1997; Stame 2004; Chen 1990). By surfacing the assumptions or rationale underlying a program, this approach can guide evaluation design and interpretation of results. Program theory can be developed by articulating the mental models of program staff or other stakeholders (e.g., participants), deductively through program documentation, or inductively through observation (Funnell and Rogers 2011). Program staff, evaluators, participants, funders, or other stakeholders may be involved in development of program theory. Diagrams 
such as logic models are sometimes used for communicating program theory (Funnell and Rogers 2011).

The field of program evaluation has seen a call for "complexity-aware" strategies for theory-based evaluation (Douthwaite and Hoffecker 2017; Douthwaite et al. 2017; Britt and Patsalides 2013). These approaches would address the need to account for complex dynamic processes affecting program outcomes at multiple scales. Some examples of causal-loop diagrams used for this purpose exist in the literature (Biroscak et al. 2014; Hassmiller Lich et al. 2017; Renmans, Holvoet, and Criel 2020). Guidelines and strategies for developing complexityaware program theory, however, have not been developed. Because causal-loop diagrams visually communicate information about an individual or group's mental model, they are well suited for illustrating qualitative data for evaluation or research (Yearworth and White 2013).

Interviews have long been used to inform the development of causal-loop diagrams, although the exact methods for gleaning causal information from qualitative data have not always been specified (Luna-Reyes and Andersen 2003). Model development, particularly for models illustrating social dynamics, is seen as a largely interpretive process dependent on modeler skill (Sterman 2000). Systematic methods for generating causal-loop diagrams from qualitative data would add transparency and rigor, therefore broadening and strengthening potential uses for the approach. Kim and Andersen (2012) presented a 
procedure based on grounded theory for coding qualitative data for causal information and constructing causal-loop diagrams from those links. The method introduced a way to track causal links to specific parts of the data and made the process more transparent, but at the expense of considerable time and effort by the modeler (Turner, Kim, and Andersen 2013). Their process also relies strictly on coding for causal links, which makes identification of feedback dynamics in source text difficult because larger causal structures (such as feedback loops and archetypes) are often communicated implicitly. Several subsequent studies (Turner et al. 2014; Biroscak et al. 2014; Valcourt et al. 2020; Eker and Zimmerman 2016) have sought to streamline or improve upon the process presented by Kim and Andersen (2012), but the same basic limitations remain.

Adding transparency and rigor to interview-based methods of model development shifts the balance of power from the modeler to the participant (Kim and Andersen 2012), thereby creating an opportunity for these methods to be used for participatory modeling. In participatory modeling, the mental model of an individual or group is represented through an iterative process facilitated by skilled modelers (Hovmand 2014; Mendoza \& Prabhu 2006; Richardson et al. 1989; Stave 2010; Vennix 1999). Mental models have been defined as internal cognitive representations of external reality (Jones et al. 2011; Schaffernicht and Groesser 2011). They reflect the assumptions or lay theories that underlie people's understanding of how the world works. A model is always a reflection of the mental model of the person or group of people that created it, according to 
some systems scientists (Meadows 2008; Senge 2010). Participatory methods therefore provide a way for models to represent the mental models of participants.

Group model building is the most common form of participatory modeling and a considerable body of knowledge about strategies for this approach have emerged (Hovmand 2014; Hovmand et al. 2012; Rouwette et al. 2002; Vennix and Gubbels 1992). In group model building, modeler-facilitators guide a group of people through a synchronous, typically in-person process of developing a systems model that reflects a shared understanding of a certain problem or issue. This approach can build group rapport and shared understanding among group members (Rouwette et al. 2002), but can be logistically challenging to arrange and can inadvertently exclude certain participants (Valcourt et al. 2020). Iterative, interview-based strategies for engaging participants may increase the diversity of voices included in modeling and enable more flexible options for asynchronous, distanced engagement. Such strategies could be used to strengthen simulation modeling or group model building, or used as a standalone participatory approach.

The research presented in this dissertation responds to the need for further developing methods for developing and using causal-loop diagrams, with a focus on applications in qualitative research and program evaluation. 
1.2. Research questions, methods, and papers

This dissertation addresses two primary research questions, organized into three distinct but related papers. To respond to the call in the evaluation literature for better ways to account for complexity in theory-based evaluation, the first paper (Chapter 2) addresses the following question:

1. How have causal-loop diagrams been used to describe and analyze 'complexity-aware' program theory?

a. Why do evaluators choose this approach?

b. How have these diagrams been developed?

c. What are the strengths and limitations of this approach?

d. How might the use of causal-loop diagrams for complexity-aware program theory be strengthened through alignment with system dynamics best practices?

To address the need for methods of systematically generating causal-loop diagrams from qualitative data, this research also considered the following question:

2. How can interviews be designed, conducted, and analyzed to identify and diagram participant mental models?

a. How can existing methods of generating causal-loop diagrams from qualitative data be improved to be more time efficient, robust, and inclusive of larger causal structures communicated implicitly?

b. How can interviews be designed to produce data suitable for this type of analysis?

The paper in Chapter 3 addresses these questions by summarizing prior methods for gleaning causal-loop diagrams from qualitative data and presenting a streamlined process centered on close analysis of source text for implicitly communicated causal structures and the use of software. The paper in Chapter 4 
draws from qualitative research, system dynamics, and realist interviewing to propose an iterative interview-based approach to mapping mental models using strategies tailored to identifying causal structures as outlined in Chapter 3. Figure 1 illustrates the connections between the content of the three papers using a diagram of the interview-based process outlined in Chapter 4.

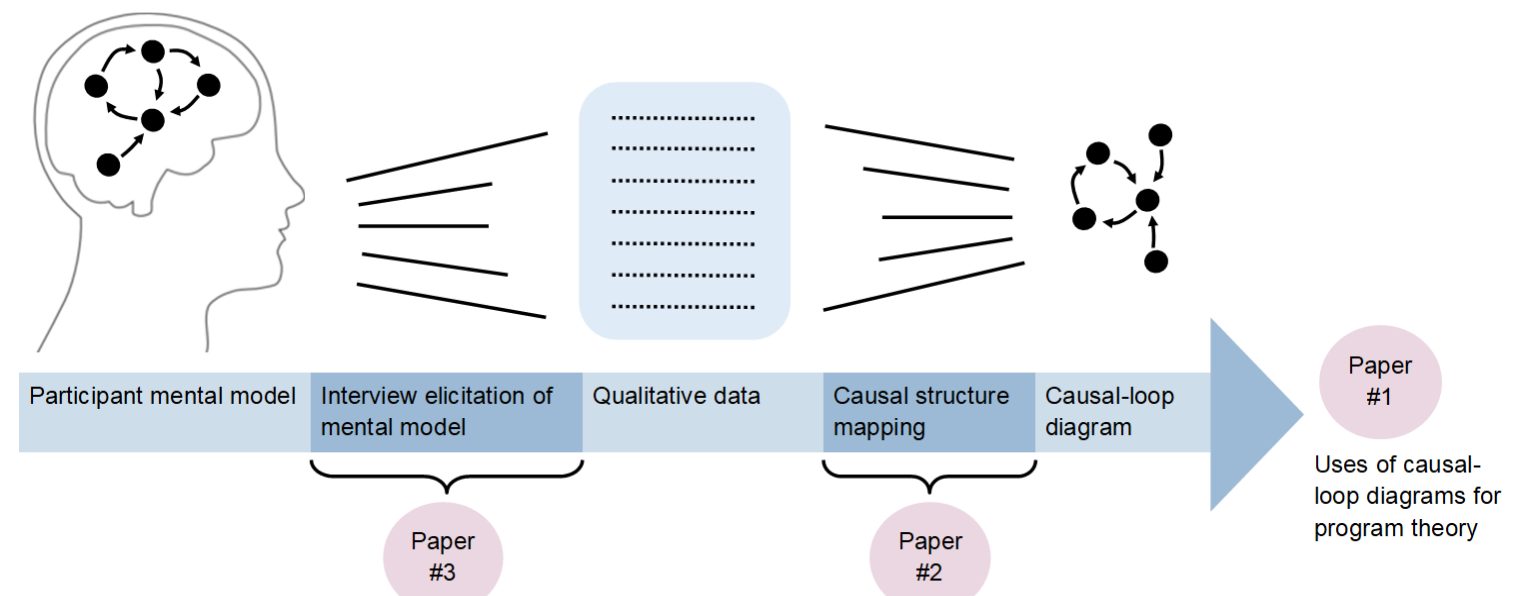

Figure 1. Dissertation papers mapped onto components of interview-based modeling approach

This dissertation sits at the nexus of qualitative research, system dynamics, and evaluation. Figure 2 illustrates the primary fields from which these papers draw. Interview data collected as part of an ongoing implementation science study were used to illustrate methods presented in Papers \#2 and \#3, but the implementation science field—which studies the uptake of evidence-based practice in clinical settings (Baur et al. 2015; Lobb and Colditz 2013)—did not directly shape methods design. 


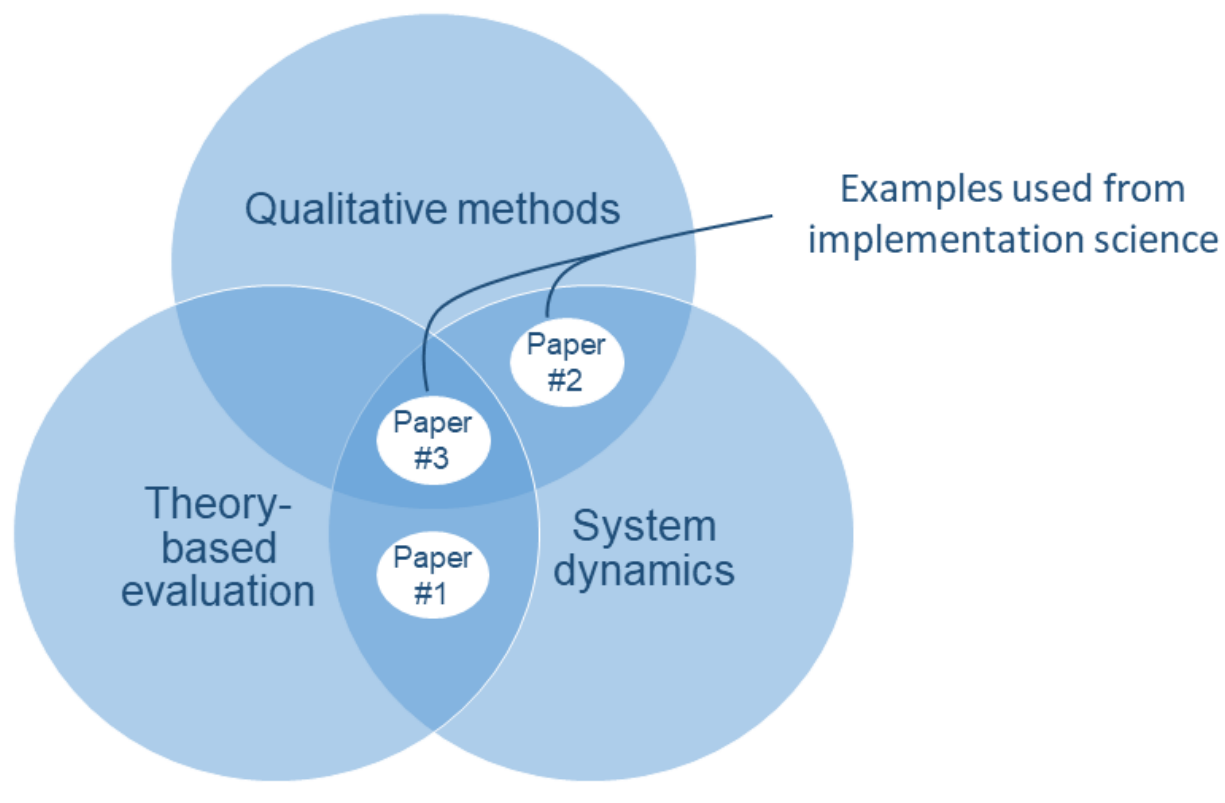

Figure 2. Three dissertation papers situated within fields of inquiry.

\subsection{Significance}

This research offers methodological innovations in two areas: (1) the generation of causal-loop diagrams from qualitative data and (2) strategies for collecting qualitative data suitable for this sort of analysis. A focus on transparent, systematic strategies for mapping individuals' mental models addresses a known need in the system dynamics literature and opens the door to broader applications in qualitative research and program evaluation. The generation of systems models from qualitative data has potentially far-reaching implications for scientific research in an era in which technological capacities for text mining using natural language processing are steadily growing. 
By examining how causal-loop diagrams are used to depict program theory, this research also explores a possible application for this interview-based modeling approach. Addressing the need for complexity-aware approaches to evaluation and research is critical for understanding pressing social challenges.

\subsection{Causal-loop diagram notation}

Causal-loop diagrams have a simple system of notation that enables the communication of a large amount of causal information (Sterman 2000). An individual link or edge in a causal-loop diagram is equivalent to the following construction: An increase in Variable A causes an increase (or decrease) in Variable $B$. This relationship is represented using a unidirectional arrow with a valence (see Figure 3). Causal-loop diagrams can therefore communicate specific causal claims (e.g., "An increase in participation in mentorship programs results in an increase in youths' self-esteem") without requiring specific equations to quantify that relationship. 


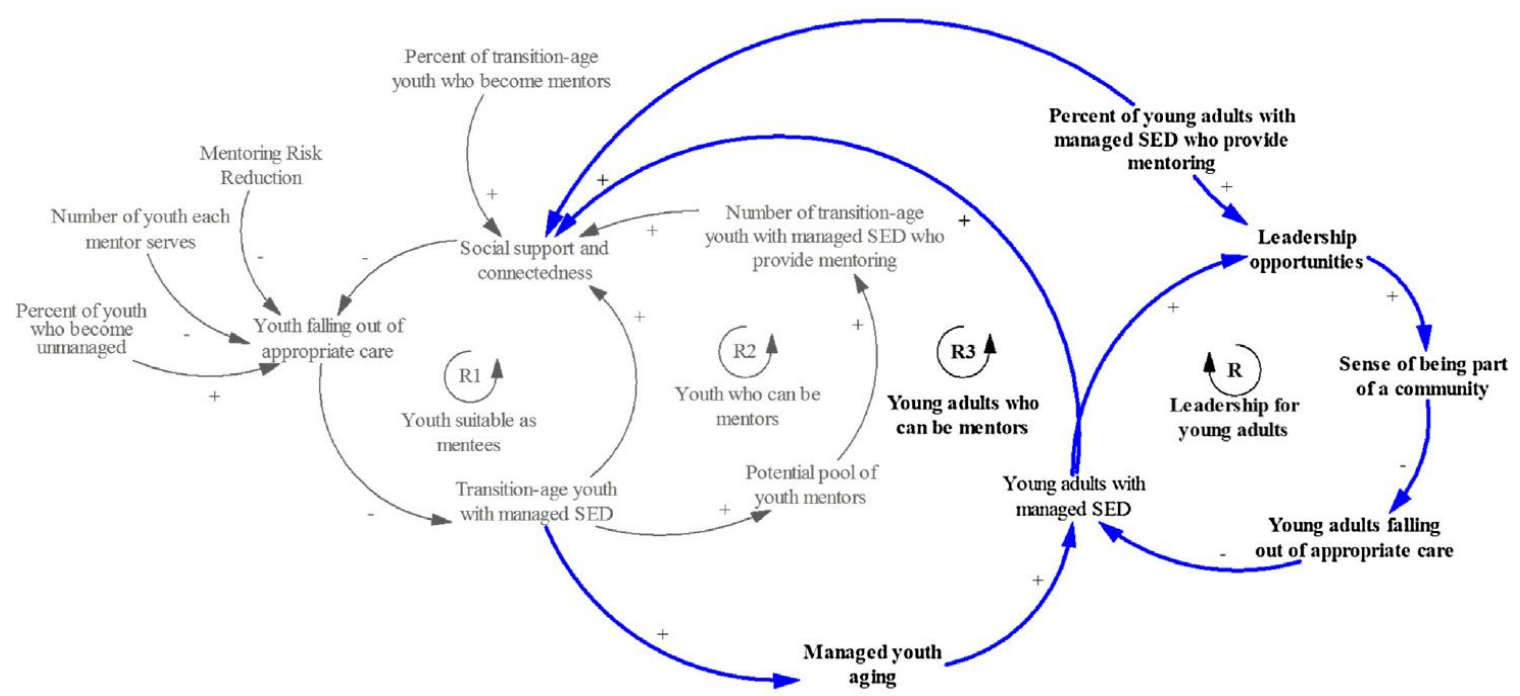

Figure 3. Example of causal-loop diagram used for complexity-aware program theory. From Hassmiller Lich et al. 2017. Original caption: "Causal loop diagram (CLD) expanding Fig. 4, which described the effects of peer mentors on increasing engagement among mentees, to include additional important and feasible constructs from GCM that could strengthen the effects of mentoring programs (R1 and R2 in light gray were previously described; R3 and [R], indicated with heavy arrows, are new). This diagram represents a complexity-aware theory of change, documenting a larger set of interconnected leverage points at which synergistic intervention could be targeted and evaluated during strategic planning."

Feedback loops take two forms: reinforcing and balancing (see Figure 4).

Reinforcing feedback describes exponential growth or decline and is commonly described as a "vicious" or "virtuous" cycle in which effects ultimately amplify their causes. Balancing feedback describes regression toward a set point or stable state and is a source of stabilization in nonlinear systems. 


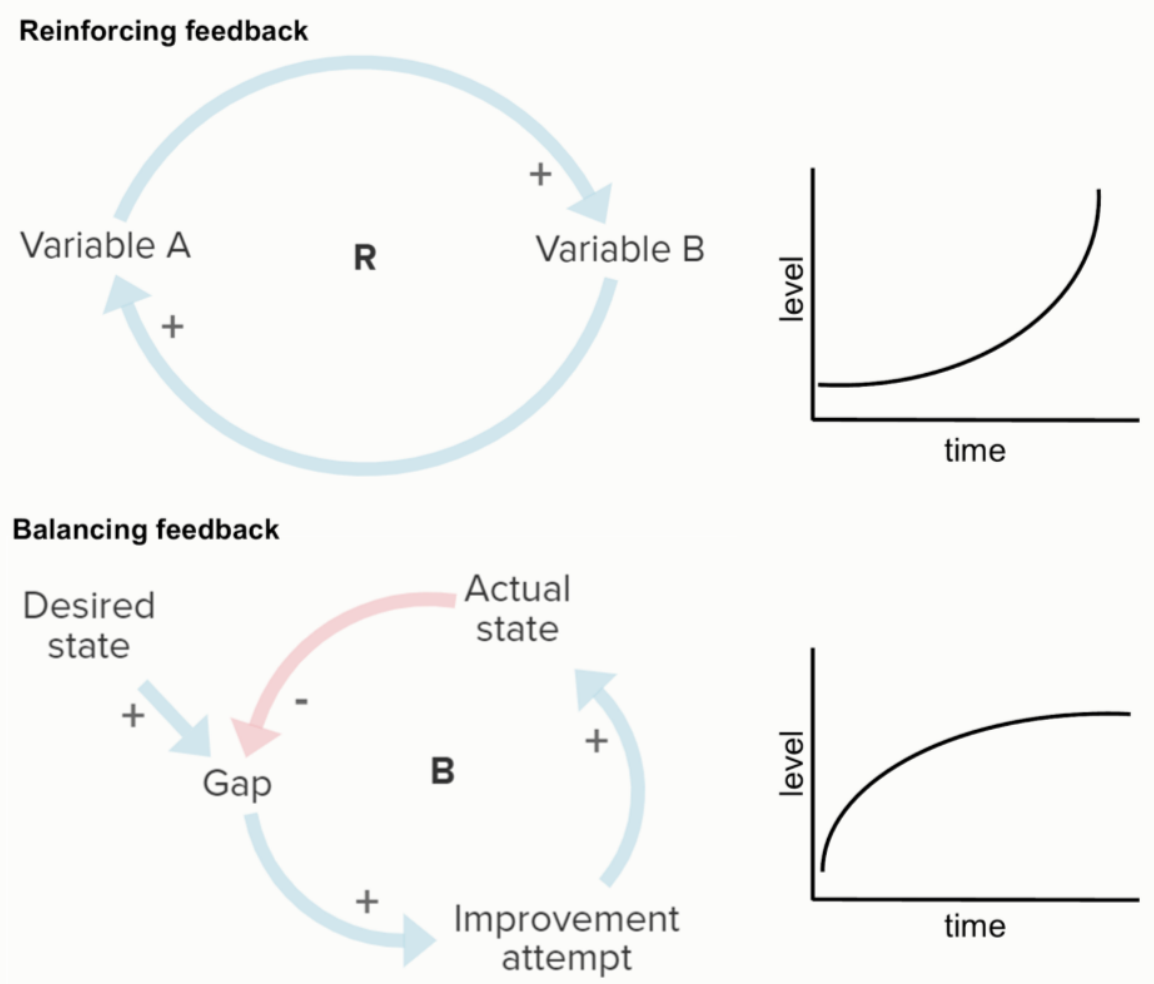

Figure 4. Generic structures of reinforcing and feedback loops with corresponding graphs of behavior over time.

This basic notation is able to summarize a wide variety of causal statements in a compact form, and can depict information from different sources in a single diagram.

\subsection{Terminology}

Some of the terminology used in system dynamics and program evaluation can be confusing due to different uses for the same term or multiple terms for the same concept. Thus, I clarify here how I use certain key terms: System dynamics is a field of study that encompasses simulation modeling (also known as computational system dynamics or numeric modeling), qualitative approaches like causal-loop diagramming (also called systems mapping), and participatory 
approaches like group model building (Hovmand 2014; Hovmand et al. 2012; Rouwette et al. 2002; Vennix and Gubbels 1992). Systems model is a general term that includes any kind of diagram or simulation designed to represent a target system; I most often use it here to refer to the causal-loop diagrams that are the topic of this research. I use the term causal structure to refer to causal links, feedback loops, and archetypes found in causal-loop diagrams or simulation models. Systems science is a broader field of study that includes system dynamics, systems theory, and other modeling approaches (e.g., agentbased simulation) (Mobus and Kalton 2015; Wakeland 2014). The term systems thinking is loosely defined but refers generally to the use of key concepts from systems science as heuristics or approaches in applications such as management or community engagement (Senge 2010; Stroh 2015; Zurcher, Jensen, and Mansfield 2018). Complexity science is an area of study focusing primarily on the identification of universal properties of complex systems (Mitchell 2009) that has different origins but considerable overlap with systems science. The adjective complexity-aware is newer and broadly defined refers to approaches in evaluation that incorporate nonlinear interactions between variables and account for multiple levels of analysis to understand complex interventions and environments.

In the field of program evaluation (also called evaluation), a wide variety of overlapping terms are used to describe ways in which programs, policies, or interventions are intended to make a difference. In this dissertation, I use the 
definition of program theory from Funnell and Rogers (2011): "an explicit theory or model of how an intervention, such as a project, a program, a strategy, an initiative, or a policy, contributes to a chain of intermediate results and finally to the intended or observed outcomes." Program theory can be described narratively or visually. The most common visual depiction of program theory is the linear "pipeline" logic model (Funnell and Rogers 2011; Kellogg Foundation 2004). The use of the term logic model in the evaluation literature sometimes refers specifically to pipeline logic models and sometimes more broadly to visual depictions of program theory (Funnell and Rogers 2011). In this research I avoid use of the general term logic model to avoid confusion; I refer specifically to pipeline (or "standard") logic models as part of the broader category of diagrams describing (or depicting) program theory. This wording is somewhat cumbersome, but hopefully more precise.

The next three chapters of this dissertation contain manuscripts of the three papers outlined in section 1.2. Chapter 2 presents results of a systematic review of evaluation studies that used causal-loop diagrams for complexity-aware program theory. Chapter 3 builds on prior research to outline a procedure for systematically generating causal-loop diagrams from qualitative data. Chapter 4 presents strategies for collecting data suitable for this analysis as part of an interview-based modeling approach. Chapter 5 synthesizes contributions to knowledge, implications, limitations, and future research. 
2. Paper \#1: Mapping complexity-aware program theory with causal-loop diagramming: A systematic review of mixed-method evaluation studies Target journals: Evaluation; Evaluation and Program Planning

\subsection{Abstract}

There has been a call in the evaluation literature for methods for developing and diagramming program theory that properly accounts for complexity. Causal-loop diagramming, a method from the interdisciplinary field of systems science, has begun to be used for this purpose, but its suitability has not been systematically explored. In this systematic review, the use of causal-loop diagramming is examined in 13 evaluation studies. Features of the diagrams, development methods, analysis or use, and identified strengths and limitations of the approach are summarized and compared. Several ways in which best practices from system dynamics could inform use of causal-loop diagrams for theory-based evaluation are identified: centering the problem, matching model structure to system behavior, using participatory methods to reflect stakeholder mental models, and including causal-loop diagramming early in program development.

\subsection{Introduction}

Funnell and Rogers (2011) define program theory as "an explicit theory or model of how an intervention, such as a project, a program, a strategy, an initiative, or a policy, contributes to a chain of intermediate results and finally to the intended or observed outcomes." Program theory describes how a program activates or influences the central processes or drivers by which change comes about at 
various levels (Funnell and Rogers 2011). Theory-based evaluation uses this approach to aid program development, monitoring, and evaluation (Weiss 1997; Stame 2004). Descriptions of program theory can be in the form of a narrative or a diagram, such as a logic model.

\subsubsection{Standard logic models and diagrams of program theory}

The standard format of a logic model is a linear "pipeline" diagram featuring program inputs, activities, outputs, outcomes, and impact (Funnel and Rogers 2011, Kellogg Foundation 2004). Figure 5 shows the basic structure of this type of logic model, reproduced from a commonly cited guide to creating and using logic models (Kellogg 2004). Other logic model guides (Innovation Network n.d.) include places along the margin to describe the problem, situation, or assumptions underlying the program, but the basic structure is the same.

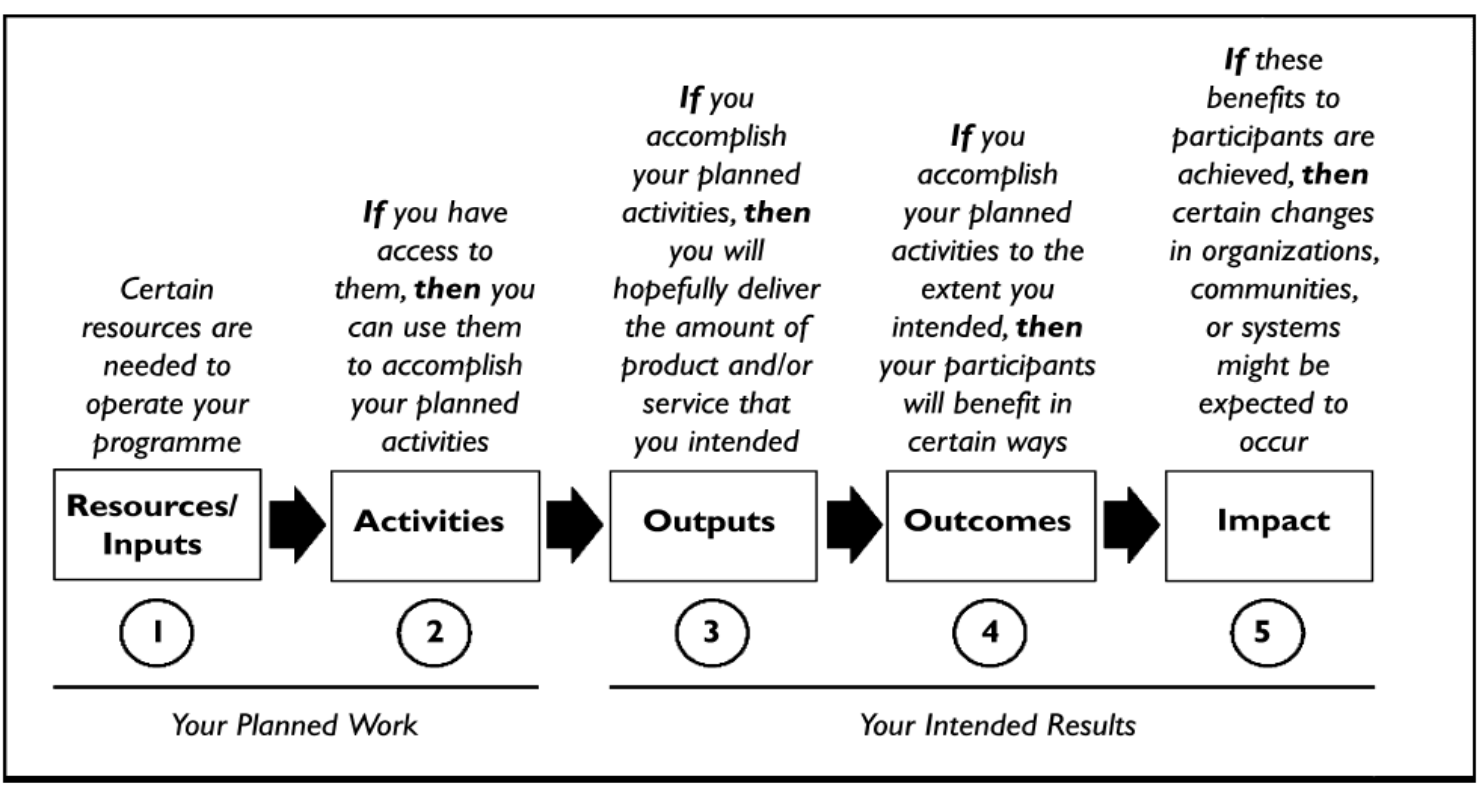

Figure 5. Basic format of a "pipeline" logic model. Reproduced from W.K. Kellogg Foundation 2004. 
The pipeline logic model clearly outlines the inputs and intended effects of a program - what is supposed to happen if everything goes according to plan. The simple format and ubiquity of this diagram mean that audiences are likely to understand it without much additional explanation. The pipeline logic model is widely used in program evaluation, and is also widely criticized (Dyehouse et al. 2009; Funnell and Rogers 2011; Miller 2013; Rogers 2008). Miller (2013) argues that the "dynamic character of practice" is lost via the "linear and mechanistic" format of logic models, and that contingencies and interrelationships are not well explained. Funnell and Rogers (2011) say that the pipeline logic model can be a useful starting point, but can entrench an "oversimplified and unhelpful" view of the program. Dyehouse and colleagues (2009) say that the standard logic model format is inadequate for capturing complex dynamics. When used to describe a complex situation, a simple logic model can cause its users to overstate the causal contribution of the intervention (Hawe 2015; Rogers 2008).

By starting with the program and its immediate inputs and specifying what happens from that point, the standard pipeline logic model can describe what is supposed to happen as a result of the program, but it is limited in its ability to describe how change comes about. Approaches that compartmentalize underlying dynamics are referred to as "black box" approaches because how inputs are turned into outputs is not made clear (Harachi et al. 1999). It is against this backdrop that calls for approaches incorporating complexity have been made. 
To address the shortcomings of overly linear logic models, a variety of new types of visual representations of program theory have emerged (Hebbard 2010; Mason and Barnes 2007; Parsons 2007; Wright and Wallis 2019). The standard pipeline logic model has been adapted in recent years to include variables related to problem or context, as well as relationships between variables (Ebenso et al. 2019; Jones et al. 2019; Renger et al. 2019). Some of these approaches include aspects of environmental influences, political context, other initiatives, and conditions for success, but the consideration of these factors varies. The Systems Evaluation Protocol includes what the researchers term "pathway" models, which is an adapted logic model that specifies connections between activities and outcomes, and minimizes the role of inputs, assumptions, and context (Hebbard 2010; Trochim et al. 2016). Douthwaite and Hoffecker (2017) utilize a causal model in their theory of change, which allows for a focus on how impact is achieved. Other researchers have also made attempts to include aspects of complexity, like feedback loops, in their diagrams (Grammatikopoulos 2012).

A specific method for developing and diagramming theories of change has emerged under the capitalized name Theories of Change ${ }^{1}$ (Clark 2012). According to Clark (Clark 2012), Theory of Change is "a representation of how

\footnotetext{
${ }^{1}$ The Theory of Change referenced here is outlined at www.theoryofchange.org and is distinct from Theories of Change as used by Funnell and Rogers (2011), which pertain to research-based theories describing human behavior.
} 
and why a complex process will succeed under specific circumstances" that consists of outcomes, interventions, assumptions, rationales, indicators, and narrative. The approach is participatory and involves working backward from a common vision for the outcomes. The resulting diagram is intended to be a "living" document that changes in accordance with new information. Several examples of this sort of diagram can be found in Appendix A.

Within the evaluation field, numerous other examples can be found of idiosyncratic mapping schemes designed mostly by independent consultants. These methods often mention "systems" or "complexity" but are largely not directly adapted from standard systems methods. One such example is the "systemigram", which is a visual diagramming method accompanied by a system narrative (McDermott, Nadolski, and Sheppard 2015).

Another approach presented by Wright and Wallis (2019) is integrative propositional analysis, which is presented as "an emerging method for rigorously and objectively evaluating the potential usefulness of conceptual systems such as theories and policy models." Also referred to as causal knowledge mapping, this approach is very similar to causal-loop diagramming in form and involves diagramming propositions and connecting them with arrows indicating causal relationships (Houston, Wright, and Wallis 2017; Wright and Wallis 2019). The number of concepts in the diagram is then counted and termed the diagram's "complexity." The number of "concatenated" concepts (concepts with two or more 
arrows pointed to them) are then tallied. The number of concatenated concepts is then divided by the number of concepts to find the "systemicity." While this approach identifies itself as being adapted from systems thinking, the two metrics it proposes for "complexity" and "systemicity" do not have any foundation in established systems science literature or practice.

The emergence of new and idiosyncratic systems mapping approaches indicate that the program evaluation community has a high degree of interest in developing visual representations of complex aspects of programs, but is largely unfamiliar with the standards of practice in systems mapping and modeling. Systems science literature and practitioners have a wealth of knowledge about best practices for mapping complex systems that are rooted in established theory and that could inform the development of hybrid methods.

The evaluation literature has seen increasing calls for "complexity-aware" monitoring and evaluation (Britt and Patsalides 2013; Douthwaite et al. 2017; Douthwaite and Hoffecker 2017; Mayne and Stern 2013; Patton 2010; PazYbarnegaray and Douthwaite 2017; Rogers 2008; Stame 2004; van Mierlo et al. 2010), which is situated in a broader literature applying concepts from complexity and systems science to evaluation (Forss, Marra, and Schwartz 2011; Gates 2016; 2017; Mowles 2014; Patton 2010; Reynolds et al. 2016; Williams and Hummelbrunner 2010; Williams and Imam 2007; Wolf-Branigin 2013). A complexity-aware approach is intended to allow for more responsive and 
adaptive learning alongside program operations (van Mierlo et al. 2010; Douthwaite and Hoffecker 2017). Despite a fair amount of discussion of complexity in the evaluation literature, a consensus has not emerged regarding what constitutes a complexity-aware approach to program theory.

Causal-loop diagramming — a method from systems science—has begun to be used to describe program theory, but its effectiveness has not been systematically studied.

\subsubsection{Causal-loop diagramming}

System dynamics is an approach for mapping and modeling complex systems that was developed in the 1950s and has been used in industry and research to address problems in areas as diverse as epidemiology, business operations, ecology, biomedicine, and economics (Forrester, 1993; Homer \& Hirsch, 2006; Sterman, 2000). System dynamics models consist of causal relationships between variables and are designed to account for nonlinear feedback relationships. The simplest form of these models are causal-loop diagrams, which are word-and-arrow diagrams showing the unidirectional causal relationships that make up reinforcing and balancing feedback loops (Sterman 2000).

Because its basic unit is a directed arrow with positive or negative valence, causal-loop diagramming also provides more information than non-causal 
network or concept diagrams that only show the existence of relationships (see Figure 6).

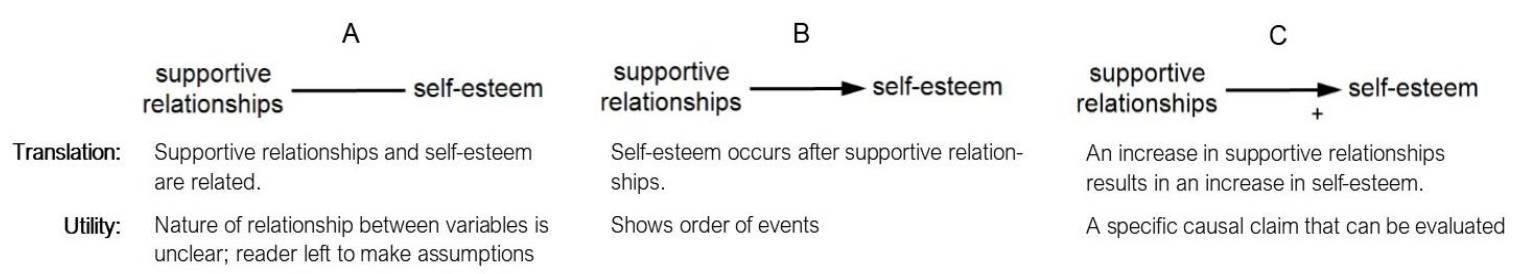

Figure 6. Comparison of diagram connection types. Figure $6 \mathrm{~A}$ shows an example of non-directed connection that indicates the existence of a relationship; Figure $6 \mathrm{~B}$ is an example of directed connection that indicates order of events or possible causal relationship; Figure $6 \mathrm{C}$ shows an example from causal-loop diagram that indicates a causal claim.

Causal-loop diagrams are often used as an initial step in building a computational system dynamics model, which operationalizes the relationships between variables featured in causal-loop diagrams to enable generation of graphs over time for key system variables (Sterman 2000). These models, also known as simulation models, provide a more robust way of exploring congruence to real data, but require significantly more data and resources to build (Sterman 2000). Due to their simpler visual layout, causal-loop diagrams are often used to describe feedback relationships when simulation modeling is not necessary or feasible. It should be noted that the term system dynamics is used to refer to the field of study that includes both causal-loop diagrams and simulation models, among other methods.

The use of system dynamics in evaluation has been discussed (Grizzle and Pettijohn 2002; Grove 2015; Hassmiller Lich et al. 2017; Burke 2006), but has not yet gained wide use. Renmans and colleagues (2017) argue that causal-loop 
diagrams are well suited for diagramming program theory because they can be used to visualize assumptions embedded in mental models—individuals' internal representations of how the world works (Jones et al. 2011) — which can lead to insight about the behavior of a system and its agents. Creating a causal-loop diagram can aid in the development of program theory and hypotheses that could be explored through theory-driven evaluation, according to the researchers. To examine how evaluation studies have used causal-loop diagramming, this article provides a systematic review of studies taking this approach. Features of the diagrams, development and analysis methods, and strengths and weaknesses identified by the evaluators are described. Ways in which alignment with best practices from system dynamics could improve this approach are presented.

\subsection{Methods}

To identify ways in which causal-loop diagrams are used and conceptualized for program theory, a systematic review of peer-reviewed and gray literature was conducted. The methods used in this review were adapted from the standards for qualitative systematic literature reviews outlined by Green and colleagues (2001). Sources included in the review were required to use causal-loop diagramming to describe how a specific program or intervention was thought to affect change in a certain context. Studies that identified only potential interventions (which encompasses a large number of standard system dynamics studies) were excluded from the review. 
A search of relevant peer-reviewed databases (e.g., Google Scholar, PsychINFO, World of Science) and the internet was used to locate suitable publications using search terms pertaining to system dynamics (e.g., causal-loop diagram) and program theory (e.g., theory of change) (see Appendix B for more details about the search strategy). Grey literature was searched to be inclusive of evaluation reports and other web-based resources used by the program evaluation field. Abstracts were screened to identify sources that utilized a causal-loop diagram for the purpose of program theory. Sources were most commonly excluded because they did not describe an evaluation of a program or intervention; many of these excluded studies used causal-loop diagramming for needs assessment or other exploratory endeavors (Hassmiller Lich et al. 2017; Brennan et al. 2015; Munro 2010). Some excluded studies outlined protocols or guidelines for using causal-loop diagramming but did not include evidence regarding their effectiveness in an actual program and were therefore excluded from the sample (Tobin et al. 2019; Lee and et. al 2016; O'Connell et al. 2016). Studies that used system dynamics to evaluate the effect of policies (Homer et al. 2009) were also excluded.

A snowball method was used in which sources cited in reviewed publications that fit the review criteria were also included. Several sources identified for inclusion in the review pertained to the same study activities. These articles were lumped together for the purpose of analysis. Figure 7 illustrates the process of review and selection. 


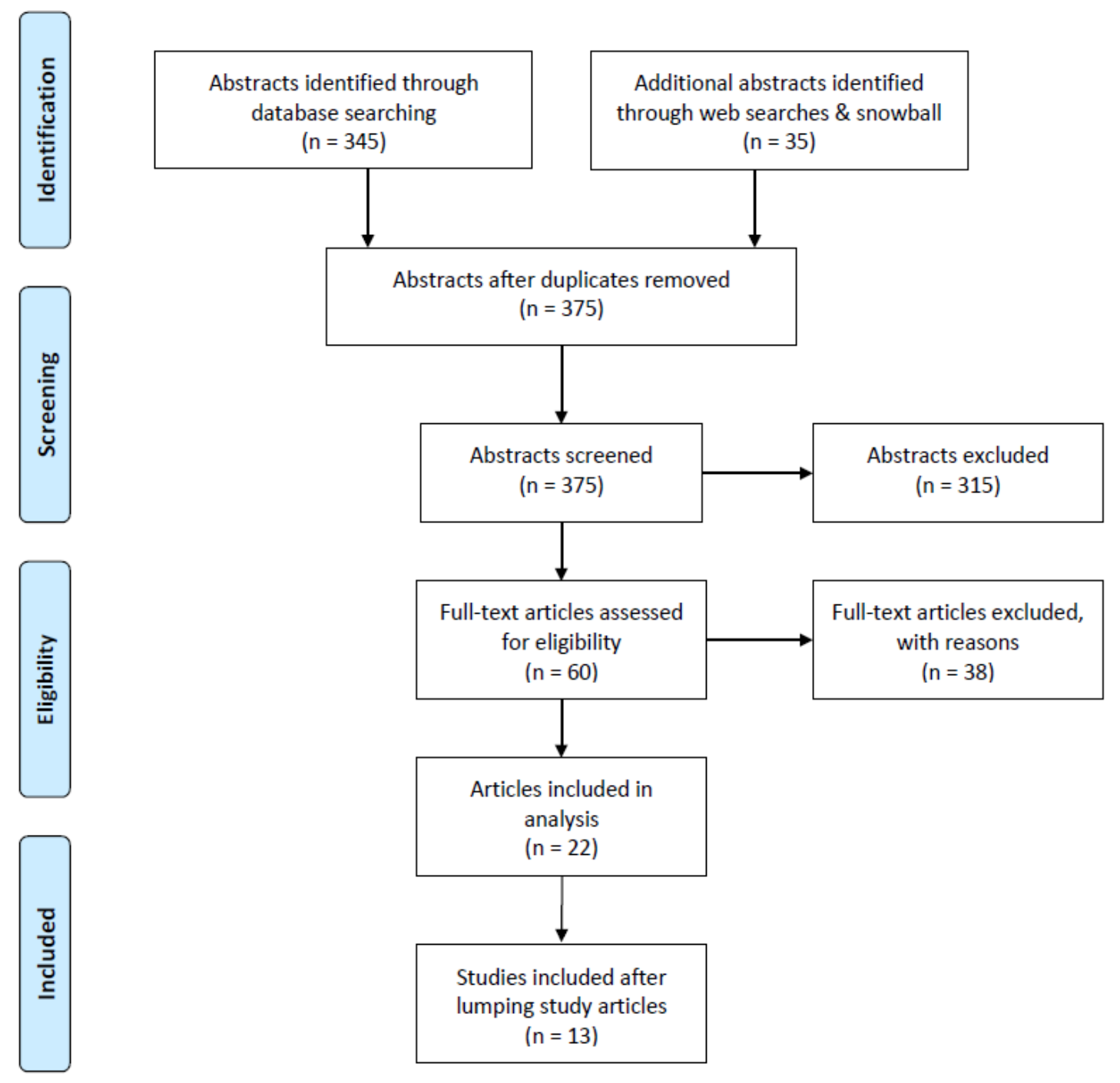

Figure 7. Process of article selection for review. Format source: http://prisma-statement.org/

Included studies were reviewed to identify the topic and location of the programs evaluated, reasons for using causal-loop diagramming, diagram features, methods of diagram development and use, and strengths and limitations of causal-loop developing identified by the researchers. Tables with this data were prepared to facilitate comparison and summary, following Green and colleagues 
(2001). One analyst familiar with causal-loop diagramming and program evaluation conducted this review.

\subsection{Results}

The search strategy and review located a total of 22 articles related to 13 unique studies using causal-loop diagrams used for program theory in evaluations. While both peer-reviewed and gray literature sources were included in the review, the final sample consisted of only peer-reviewed articles. The articles were published between 2008 and 2020 and span a variety of program contexts, including economic development, health services, education, and social services. Five studies took place in the United States; four studies described programs in African countries; two were based in Europe; and one study each was conducted in Australia and Afghanistan. Several studies following the evaluation guidance of the international One Health initiative were grouped together for analysis. Publications from two other studies were similarly grouped because they described the same evaluation.

While many of the articles cite foundational system dynamics or systems thinking literature (e.g., Sterman 2000; Meadows 2008), only three articles put their projects in the context of the literature on systems in evaluation. Only one of the included studies referenced the literature on complexity-aware program theory. Only five studies cited other included studies; Dyehouse and colleagues (2009) was cited three times, Fredericks and colleagues (2008) was cited twice, and 
Sarriot and colleagues (2015) was cited once. In other words, the researchers appeared to have largely arrived at similar methods independently from one another while seeking effective ways to conduct evaluation, rather than borrowing from other studies. Table 1 summarizes the topics of the causal-loop diagrams by study, how they were designed, and how they were analyzed.

Studies are grouped according to diagram development approach (participatory modeling, analysis of prior evaluation data, and evaluator led / unknown).

Table 1. Evaluation studies utilizing causal-loop diagrams for program theory

\begin{tabular}{|c|c|c|c|}
\hline Study ${ }^{1}$ & Topic of diagram & Diagram development & Analysis and use \\
\hline \multicolumn{4}{|c|}{ Participatory modeling } \\
\hline $\begin{array}{l}\text { Renmans et } \\
\text { al. } 2020 \\
\text { and } \\
\text { Renmans et } \\
\text { al. } 2017\end{array}$ & $\begin{array}{l}\text { A series of causal- } \\
\text { loop diagrams } \\
\text { describing context, } \\
\text { mechanisms, and } \\
\text { outcomes of a } \\
\text { performance-based } \\
\text { financing intervention } \\
\text { in the Ugandan health } \\
\text { care sector created } \\
\text { as part of a realist } \\
\text { evaluation }\end{array}$ & $\begin{array}{l}\text { An initial model of the health } \\
\text { system was created based on } \\
\text { key informant interviews, } \\
\text { scientific literature, and policy } \\
\text { documents. Program theory } \\
\text { diagrams describing how the } \\
\text { intervention acted on the system } \\
\text { were created based on } \\
\text { additional interviews and } \\
\text { literature review. }\end{array}$ & $\begin{array}{l}\text { Diagrams were } \\
\text { segmented, revised, and } \\
\text { context, mechanisms, and } \\
\text { outcomes were identified } \\
\text { in the diagram as part of a } \\
\text { realist evaluation. } \\
\text { Diagrams were then } \\
\text { merged. Feedback loops } \\
\text { were identified using } \\
\text { software. Archetypes were } \\
\text { identified from those loops. }\end{array}$ \\
\hline & $\begin{array}{l}\text { A series of causal- } \\
\text { loop diagrams } \\
\text { describing a program } \\
\text { to teach community }\end{array}$ & $\begin{array}{l}\text { The diagram was created using } \\
\text { group model building with } \\
\text { stakeholders and adhered to } \\
\text { Sterman's model building steps. } \\
\text { Transcripts from meetings, } \\
\text { interviews and mini-focus group } \\
\text { sessions were analyzed } \\
\text { according to the method outlined } \\
\text { by Kim and Anderson (2012). } \\
\text { Individual causal links were } \\
\text { identified and then assembled }\end{array}$ & \\
\hline $\begin{array}{l}\text { Biroscak et } \\
\text { al. } 2014 ; \\
\text { Biroscak } \\
2014\end{array}$ & $\begin{array}{l}\text { coalitions how to } \\
\text { apply social } \\
\text { marketing to policy } \\
\text { change in the US }\end{array}$ & $\begin{array}{l}\text { into a diagram. Multiple coders } \\
\text { were used to enhance reliability. } \\
\text { This activity was part of a } \\
\text { utilization-focused evaluation. }\end{array}$ & $\begin{array}{l}\text { Loops were identified, } \\
\text { named, and described to } \\
\text { present evaluation } \\
\text { findings. }\end{array}$ \\
\hline
\end{tabular}




\begin{tabular}{|c|c|c|c|}
\hline $\begin{array}{l}\text { Merrill et al. } \\
2013\end{array}$ & $\begin{array}{l}\text { A series of hybrid } \\
\text { causal-loop / stock- } \\
\text { and-flow diagrams } \\
\text { were used to evaluate } \\
\text { the implementation of } \\
\text { electronic health } \\
\text { information exchange } \\
\text { systems for public } \\
\text { health reporting at a } \\
\text { state health } \\
\text { department in the US }\end{array}$ & $\begin{array}{l}\text { The diagrams were created } \\
\text { through a participatory process } \\
\text { with experts and extensive } \\
\text { project documentation. Experts } \\
\text { were engaged in a group and } \\
\text { individually according to group } \\
\text { model building scripts (e.g., } \\
\text { reference behavior mode } \\
\text { identification, influence } \\
\text { diagrams, etc.). Iterative rounds } \\
\text { of model revision and feedback } \\
\text { were used to increase } \\
\text { confidence and accuracy of the } \\
\text { model. }\end{array}$ & $\begin{array}{l}\text { The diagram loops were } \\
\text { used to describe the } \\
\text { evaluation results in detail, } \\
\text { including several named } \\
\text { structures. Points of } \\
\text { leverage were also } \\
\text { identified. }\end{array}$ \\
\hline $\begin{array}{l}\text { Fredericks } \\
\text { et al. } 2008\end{array}$ & $\begin{array}{l}\text { A series of causal- } \\
\text { loop diagrams } \\
\text { describe a multi-site } \\
\text { program to provide } \\
\text { individualized } \\
\text { services to people } \\
\text { with developmental } \\
\text { disabilities in the US }\end{array}$ & $\begin{array}{l}\text { The diagram was created using } \\
\text { an iterative participatory process } \\
\text { involving stakeholders. Source } \\
\text { material included evaluation } \\
\text { findings to date, stakeholders' } \\
\text { observations of program } \\
\text { activities, and interviews with } \\
\text { program staff. }\end{array}$ & $\begin{array}{l}\text { The diagram was used to } \\
\text { inform program } \\
\text { implementation by } \\
\text { identifying certain } \\
\text { dynamics constituting } \\
\text { barriers (e.g., competing } \\
\text { goals and capacity } \\
\text { limitations in the } \\
\text { agencies). }\end{array}$ \\
\hline \multicolumn{4}{|c|}{ Analysis of prior evaluation data } \\
\hline $\begin{array}{l}\text { Owen et al. } \\
2018\end{array}$ & $\begin{array}{l}\text { A causal-loop } \\
\text { diagram describing } \\
\text { factors contributing } \\
\text { toward the success of } \\
\text { a childhood obesity } \\
\text { prevention program in } \\
\text { Australia }\end{array}$ & $\begin{array}{l}\text { The diagram was created using } \\
\text { secondary analysis of qualitative } \\
\text { interview data from a prior } \\
\text { evaluation. Researchers } \\
\text { followed Kim and Anderson's } \\
\text { method for analyzing qualitative } \\
\text { data (2012). Exogenous } \\
\text { variables were removed and the } \\
\text { diagram was edited to highlight } \\
\text { key feedback loops. Two experts } \\
\text { involved in program } \\
\text { implementation provided } \\
\text { feedback. }\end{array}$ & $\begin{array}{l}\text { Key feedback loops were } \\
\text { identified and described. }\end{array}$ \\
\hline $\begin{array}{l}\text { Okumu et } \\
\text { al. } 2016\end{array}$ & $\begin{array}{l}\text { A series of causal- } \\
\text { loop diagrams } \\
\text { describing how a pen- } \\
\text { based digital learning } \\
\text { intervention } \\
\text { influences student } \\
\text { learning in the US }\end{array}$ & $\begin{array}{l}\text { The diagrams were developed } \\
\text { by coding prior evaluation } \\
\text { interviews according to the } \\
\text { method presented by Kim and } \\
\text { Andersen (2012). }\end{array}$ & $\begin{array}{l}\text { The loops in the diagram } \\
\text { were presented alongside } \\
\text { descriptions of evaluation } \\
\text { findings. }\end{array}$ \\
\hline $\begin{array}{l}\text { Sarriot et al. } \\
2015\end{array}$ & $\begin{array}{l}\text { A causal-loop } \\
\text { diagram describing } \\
\text { the sustainability of a } \\
\text { program for } \\
\text { integrated community } \\
\text { case management of } \\
\text { malaria, pneumonia, } \\
\text { and diarrhea in }\end{array}$ & $\begin{array}{l}\text { The diagram was created using } \\
\text { prior evaluation data (individual } \\
\text { and group interview data, } \\
\text { exchanges with stakeholders, } \\
\text { and survey data). Factors were } \\
\text { categorized according to } \\
\text { domains in a conceptual } \\
\text { framework and ranked for }\end{array}$ & $\begin{array}{l}\text { The diagram was used to } \\
\text { inform participatory } \\
\text { discussions of near-term } \\
\text { scenarios; these } \\
\text { discussions further refined } \\
\text { the diagram. Results were } \\
\text { presented. }\end{array}$ \\
\hline
\end{tabular}




\begin{tabular}{|c|c|c|c|}
\hline & Rwanda & $\begin{array}{l}\text { relevance. The diagram was } \\
\text { then simplified. }\end{array}$ & \\
\hline $\begin{array}{l}\text { Kwamie, et } \\
\text { al. } 2014\end{array}$ & $\begin{array}{l}\text { A causal-loop } \\
\text { diagram describing a } \\
\text { failed leadership } \\
\text { development program } \\
\text { for district managers } \\
\text { of health systems in } \\
\text { Ghana }\end{array}$ & $\begin{array}{l}\text { As part of a realist evaluation, } \\
\text { the researchers coded } \\
\text { qualitative data, configured } \\
\text { context-mechanism- outcome } \\
\text { configurations from the data, } \\
\text { and displayed it as a causal-loop } \\
\text { diagram. }\end{array}$ & $\begin{array}{l}\text { The causal-loop diagram } \\
\text { was used in a realist } \\
\text { evaluation. Feedback } \\
\text { loops were described, } \\
\text { including loops that would } \\
\text { have been seen had the } \\
\text { intervention been } \\
\text { successful. Implicit system } \\
\text { goals were identified. }\end{array}$ \\
\hline \multicolumn{4}{|c|}{ Evaluator-led modeling / unknown } \\
\hline $\begin{array}{l}\text { Rüegg et al. } \\
2018 ; \\
\text { Rüegg, } \\
\text { Häsler, and } \\
\text { Zinsstag } \\
\text { 2018; } \\
\text { Duboz et al. } \\
\text { 2018; Hanin } \\
\text { et al. 2018; } \\
\text { Léger et al. } \\
\text { 2018; } \\
\text { Muñoz- } \\
\text { Prieto et al. } \\
\text { 2018; } \\
\text { Wilcox et al. } \\
2019 \text { (One } \\
\text { Health } \\
\text { initiative) }\end{array}$ & $\begin{array}{l}\text { Par diagram used as } \\
\text { A modified causal- } \\
\text { loop diart of an evaluation } \\
\text { framework for } \\
\text { projects in a large } \\
\text { multi-site international } \\
\text { program to reduce } \\
\text { antimicrobial } \\
\text { resistance }\end{array}$ & $\begin{array}{l}\text { The diagram is a hybrid of a } \\
\text { causal-loop diagram and } \\
\text { Ostrom's social-ecological } \\
\text { system framework (2009). } \\
\text { Diagrams describing the context } \\
\text { were developed, then variables } \\
\text { representing the interventions } \\
\text { were added. The mixed methods } \\
\text { evaluation also included a } \\
\text { separate theory of change. } \\
\text { Questions guiding diagram } \\
\text { creation were provided, but } \\
\text { participants and process for } \\
\text { diagram creation were not } \\
\text { described in detail. }\end{array}$ & $\begin{array}{l}\text { Diagrams were used to } \\
\text { understand program } \\
\text { context using four aspects } \\
\text { of relationships (Williams } \\
\text { 2016) - topology of links, } \\
\text { type of relationship, link } \\
\text { characteristics, and } \\
\text { prioritization. }\end{array}$ \\
\hline $\begin{array}{l}\text { Knai et al. } \\
2018\end{array}$ & $\begin{array}{l}\text { A causal-loop } \\
\text { diagram was created } \\
\text { to understand why a } \\
\text { public-private } \\
\text { partnership to } \\
\text { improve public health } \\
\text { failed in England }\end{array}$ & $\begin{array}{l}\text { The diagram was created from a } \\
\text { prior mixed-methods evaluation, } \\
\text { consisting of an initial logic } \\
\text { model, literature review, } \\
\text { stakeholder and informant } \\
\text { interviews, quantitative outcome } \\
\text { data, case studies, comparative } \\
\text { analyses, and media analyses. } \\
\text { Processes for generating the } \\
\text { diagram from these data are not } \\
\text { specified. }\end{array}$ & $\begin{array}{l}\text { The diagram was used to } \\
\text { inform possible sources of } \\
\text { the system's resilience. } \\
\text { Analysis methods were not } \\
\text { specified. }\end{array}$ \\
\hline
\end{tabular}




\begin{tabular}{|c|c|c|c|}
\hline $\begin{array}{l}\text { Alonge et } \\
\text { al. } 2017\end{array}$ & $\begin{array}{l}\text { A series of causal- } \\
\text { loop diagrams and } \\
\text { simulation models } \\
\text { were used to test an } \\
\text { implicit theory of } \\
\text { change for an } \\
\text { unsuccessful supply } \\
\text { side pay for } \\
\text { performance scheme } \\
\text { to improve health } \\
\text { system performance } \\
\text { in Afghanistan. }\end{array}$ & $\begin{array}{l}\text { The causal-loop diagrams were } \\
\text { created based on prior survey } \\
\text { data. Methods for gleaning } \\
\text { model components from the } \\
\text { data were not described. A } \\
\text { quantitative simulation model } \\
\text { was developed based on the } \\
\text { diagram. }\end{array}$ & $\begin{array}{l}\text { The diagram and model } \\
\text { were used to "provide } \\
\text { insights into how key } \\
\text { implementation processes } \\
\text { could influence outcomes } \\
\text { of the intervention." } \\
\text { Various scenarios were } \\
\text { explored. The study } \\
\text { concluded that the } \\
\text { intervention would likely } \\
\text { have been successful if } \\
\text { not for poor } \\
\text { implementation. }\end{array}$ \\
\hline $\begin{array}{l}\text { Mutale et } \\
\text { al. } 2017 ; \\
2016\end{array}$ & $\begin{array}{l}\text { A series of causal- } \\
\text { loop diagrams } \\
\text { describing a multi- } \\
\text { site, multi-level } \\
\text { intervention to } \\
\text { improve health } \\
\text { system effectiveness } \\
\text { in Zambia. }\end{array}$ & $\begin{array}{l}\text { The diagrams were developed } \\
\text { as part of a mixed methods } \\
\text { evaluation involving stakeholder } \\
\text { participation. The study refers to } \\
\text { WHO guidance for using } \\
\text { systems thinking to strengthen } \\
\text { health systems (de Savigny and } \\
\text { Adam 2009) but does not } \\
\text { describe model creation in } \\
\text { detail. }\end{array}$ & $\begin{array}{l}\text { The diagram was used as } \\
\text { a conceptual framework to } \\
\text { analyze study findings, } \\
\text { including intended and } \\
\text { unintended consequences. } \\
\text { Analysis was conducted } \\
\text { based on key subsystems } \\
\text { and loops were described } \\
\text { in detail. }\end{array}$ \\
\hline $\begin{array}{l}\text { Dyehouse } \\
\text { et al. } 2009\end{array}$ & $\begin{array}{l}\text { A series of causal- } \\
\text { loop diagrams } \\
\text { describing an } \\
\text { interdisciplinary } \\
\text { program to improve } \\
\text { STEM education in } \\
\text { rural middle schools } \\
\text { in the US }\end{array}$ & $\begin{array}{l}\text { The diagrams were created } \\
\text { using Cabrera's steps to using } \\
\text { systems thinking (2008) and } \\
\text { Coyle's list extension technique } \\
\text { (2004). Details about how prior } \\
\text { evaluation data were used and } \\
\text { who was involved in creating the } \\
\text { diagrams were not provided. } \\
\text { Diagrams were revised during } \\
\text { implementation. Stakeholder } \\
\text { involvement is praised, but it is } \\
\text { unclear whether it was used. }\end{array}$ & $\begin{array}{l}\text { The diagrams were used } \\
\text { as a logic model to aid } \\
\text { evaluators' thinking- to } \\
\text { identify potential solutions } \\
\text { to implementation } \\
\text { challenges. Feedback } \\
\text { loops were identified and } \\
\text { used to enhance } \\
\text { understanding of program } \\
\text { dynamics. Specific } \\
\text { methods for analysis were } \\
\text { not detailed. }\end{array}$ \\
\hline
\end{tabular}

${ }^{1}$ Articles pertaining to the same research study or program are grouped together.

\subsubsection{Reasons for using causal-loop diagramming}

The reasons for utilizing causal-loop diagramming in the evaluations were

described similarly by the authors: a desire to take a 'systems approach' that

describes relationships between context, intervention, and outcomes. Several

studies incorporated causal-loop diagramming after encountering limitations of

standard approaches. Fredericks and colleagues (2008) incorporated causal-

loop diagramming into their evaluation after encountering unexpected findings 
during program implementation. Dyehouse and colleagues (2009) switched from a standard logic model approach to a causal-loop diagram after finding the former approach lacking. Several studies (Knai et al. 2018; Alonge et al. 2017) used causal-loop diagramming in a secondary analysis of evaluation findings to understand why the intervention failed.

\subsubsection{Diagram development}

Diagrams were created using three categories of approaches: participatory modeling involving stakeholders or experts, systematic analysis of prior evaluation data, and a looser approach in which model development was led by the evaluator or not described (see Table 1). All four studies that utilized participatory approaches conducted individual interviews, while two of those studies (Biroscak et al. 2014; Merrill et al. 2013) also used group model building. These approaches were iterative and involved participants in multiple phases of diagram revision. One participatory study followed the procedure outlined by Kim and Andersen (2012) to generate causal-loop diagrams from text data gathered from meetings, interviews, and focus groups in addition to group model building.

Four studies used secondary analysis of prior evaluation data to identify diagram components. Of the four studies that used this approach, two (Owen et al. 2018; Okumu et al. 2016) followed Kim and Andersen's method. Owen and colleagues (2018) supplemented this approach by obtaining feedback about model structure from two experts. Sarriot and colleagues (2015) categorized domains identified in 
mixed methods data (individual and group interviews, stakeholder interactions, and survey data), and developed a model from those domains. As part of a realist evaluation, Kwamie and colleagues (2014) identified context-mechanismoutcome configurations by coding qualitative data, then rendered those configurations in a causal-loop diagram.

Five studies used causal-loop diagramming to analyze and communicate evaluation findings, but did not describe methods for identifying diagram components through participatory processes or systematic analysis of evaluation data. While it is possible these methods were used but not described, it may be reasonable to assume that the diagrams were created by evaluators in a more informal way based on their mental models of how the program and underlying system function and interpretation of evaluation findings.

Studies varied considerably in their use of standard processes for system dynamics model development. Only two of the studies mentioned identifying reference behavior patterns with stakeholders (Biroscak et al. 2014; Merrill et al. 2013). Dyehouse and colleagues (2009) cite a procedure outlined by Cabrera (2008) in which program components are identified prior to relationships between them, and feedback loops are identified from those relationships-a process not aligned with system dynamics best practice. The exclusion of exogenous variables found in Owen and colleagues (2018) is also not typical in system 
dynamics. Only two studies mentioned diagramming the pre-existing system or problem prior to adding variables related to the intervention.

\subsubsection{Diagram features}

The causal-loop diagrams produced by the included studies vary considerably in their degree of sophistication and adherence to the norms of system dynamics. Typically, causal-loop diagrams contain several feedback loops, as well as exogenous variables driving the system (Sterman 2000). Loops are clearly visible and labeled, and diagrams are organized to minimize overlap and clutter (see Table 7 in Chapter 3 for norms of causal-loop diagramming). The model presented by Alonge and colleagues (2017) contained only six endogenous variables, while other diagrams contained many variables, including exogenous drivers relevant to context. Several diagrams adapted the standard "word and arrow" format of the causal-loop diagram to include different font sizes or styles, arrow colors, or variable shapes. Several diagrams were hybrid causal-loop and stock-and-flow diagrams, a common practice in system dynamics. The One Health evaluations (Rüegg et al. 2018; Rüegg 2018; Duboz et al. 2018; Hanin et al. 2018; Léger et al. 2018; Muñoz-Prieto et al. 2018; Wilcox et al. 2019) followed an approach blending causal-loop diagramming, flow chart notation, and Ostrom's social-ecological systems framework (2009). Two studies (Sarriot et al. 2015; Renmans et al. 2020) labeled certain regions of their diagrams to aid comprehension. In their realist evaluation, Renmans and colleagues (2020) visually distinguished diagram regions corresponding to context, mechanisms, 
and outcomes. An earlier study from the same authors illustrating a portion of their model is shown in Figure 8 to illustrate a standard causal-loop diagram. A selection of causal-loop diagrams representing different visual formats used by studies included in this review can be found in Appendix C.

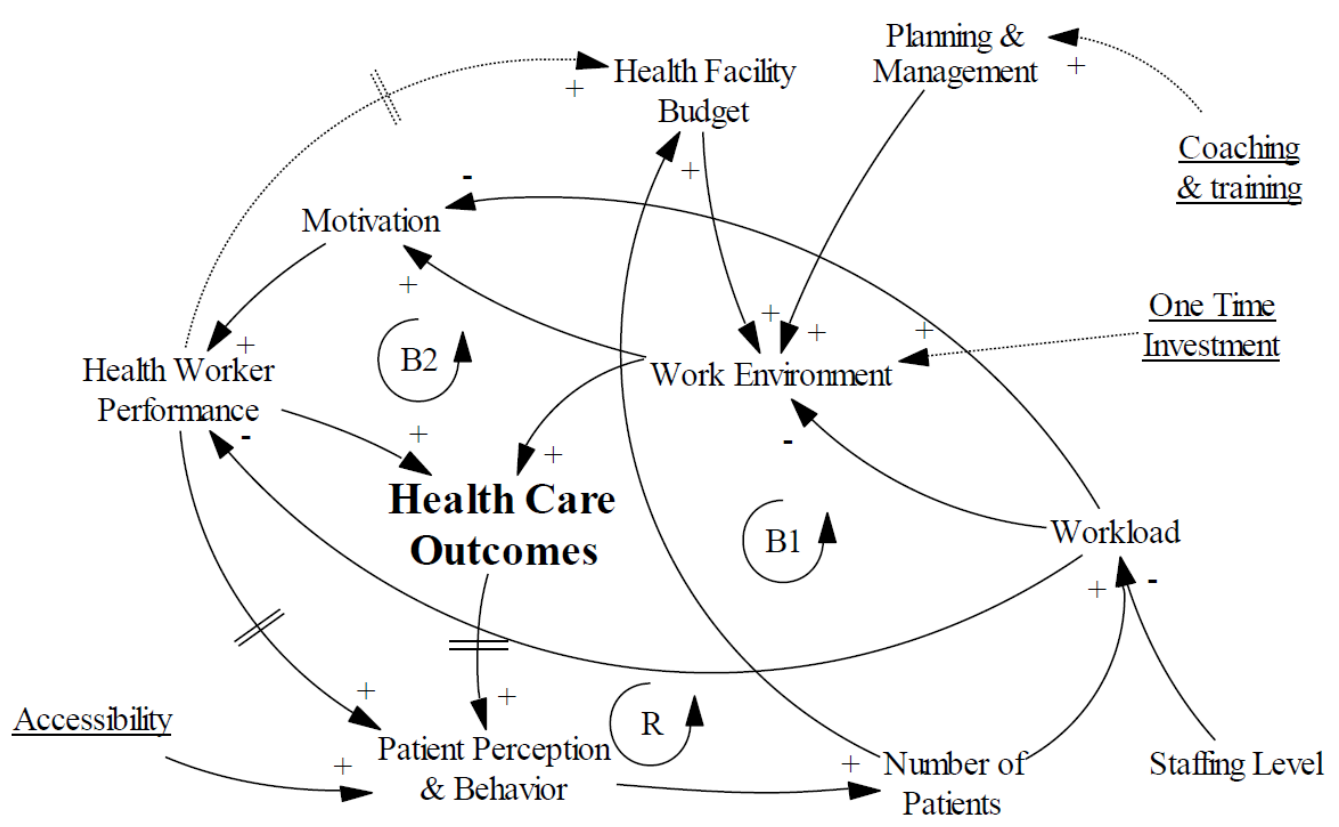

Figure 8. Example causal-loop diagram from an included study showing "growth and underinvestment" archetype. Source: Renmans et al. 2017.

\subsubsection{Diagram analysis and use}

While the diagrams were created using different methods, they were used in largely similar ways. In many of the studies, feedback loops in the causal-loop diagram were described and used to frame the presentation of qualitative evaluation findings. Renmans and colleagues (2017) identified archetypes in their diagrams. Archetypes are certain system configurations that describe situations common across different domains (Sterman 2000; Meadows 2008; Senge 2010). Two studies (Renmans et al. 2020 and Kwamie et al. 2014) used 
the causal-loop diagrams as part of a realist evaluation. In one study (Alonge et al. 2017), the causal-loop diagrams were operationalized into computational system dynamics models capable of generating estimated graphs of behavior over time for key variables. One study (Fredericks et al. 2008) used causal-loop diagramming alongside pipeline logic models, although the content between the two diagrams did not match and the role of each diagram on the research projects was not well described.

2.4.5. Identified strengths and limitations of causal-loop diagramming for program theory

Overall, the use of causal-loop diagramming was described positively by study authors.

Strengths of the method were described across several categories: understanding system components and behavior, clarifying the intervention, understanding implementation, communicating evaluation findings, increasing the validity of evaluation findings, and several other miscellaneous benefits (see Table 2). 
Table 2. Strengths of causal-loop diagramming for program theory identified in included studies.

\section{Strengths of causal-loop diagramming Source}

\section{Understand system components and behavior}

\begin{tabular}{|l|l|}
\hline Identify contextual elements & $\begin{array}{l}\text { Renmans et al. 2020; Merrill et al. 2013; Rüegg et al. } \\
\text { 2018; Mutale et al. 2017; Kwamie et al. 2014; } \\
\text { Dyehouse et al. 2009 }\end{array}$ \\
\hline $\begin{array}{l}\text { Shows the causal structure responsible for } \\
\text { behavior of outcome variables over time }\end{array}$ & $\begin{array}{l}\text { Fredericks et al. 2008; Knai et al. 2018; Sarriot et al. } \\
\text { 2015; Rüegg et al. 2018; Dyehouse et al. 2009 }\end{array}$ \\
\hline $\begin{array}{l}\text { Identify variables and relationships } \\
\text { between them }\end{array}$ & $\begin{array}{l}\text { Renmans et al. 2020; Biroscak et al. 2014; Merrill et } \\
\text { al. } 2013\end{array}$ \\
\hline Identify feedback loops & Renmans et al. 2020; Okumu et al. 2016 \\
\hline $\begin{array}{l}\text { Facilitate understanding of underlying } \\
\text { problems }\end{array}$ & Fredericks et al. 2008; Dyehouse et al. 2009 \\
\hline Identify underlying assumptions & Renmans et al. 2020 \\
\hline Clarify intervention & \\
\hline
\end{tabular}

\begin{tabular}{|l|l|}
\hline Explore what-if scenarios & $\begin{array}{l}\text { Okumu et al. 2016; Sarriot et al. 2015; Kwamie et al. } \\
\text { 2014; Dyehouse et al. 2009 }\end{array}$ \\
\hline $\begin{array}{l}\text { Identify possible unintended } \\
\text { consequences }\end{array}$ & $\begin{array}{l}\text { Merrill et al. 2013; Fredericks et al. 2008; Mutale et } \\
\text { al. 2017; Dyehouse et al. 2009 }\end{array}$ \\
\hline Identify and improve intervention & $\begin{array}{l}\text { Renmans et al. 2020; Biroscak et al. 2014; } \\
\text { Fredericks et al. 2008 }\end{array}$ \\
\hline Inform future programs & Knai et al. 2018; Owen et al. 2018 \\
\hline Understand what happened (e.g., failure) & $\begin{array}{l}\text { Alonge et al. 2017; Kwamie et al. 2014; Knai et al. } \\
\text { 2018 }\end{array}$ \\
\hline Formulate critiques of the intervention & Renmans et al. 2020; Okumu et al. 2016 \\
\hline $\begin{array}{l}\text { See intervention as acting on an existing } \\
\text { system }\end{array}$ & Renmans et al. 2020 \\
\hline Understand implementation & \\
\hline Inform implementation of the intervention & Merrill et al. 2013 \\
\hline $\begin{array}{l}\text { Identify reasons for variability in } \\
\text { implementation and the range of program } \\
\text { outcomes }\end{array}$ & Fredericks et al. 2008 \\
\hline Communicate evaluation findings & \\
\hline Visually communicate complex issues & $\begin{array}{l}\text { Renmans et al. 2020; Owen et al. 2018; Dyehouse } \\
\text { et al. 2009 }\end{array}$ \\
\hline Position findings in context for audience & Fredericks et al. 2008 \\
\hline $\begin{array}{l}\text { Summarize dynamics familiar to } \\
\text { stakeholders }\end{array}$ & Sarriot et al. 2015 \\
\hline Encourage informed decision-making & Sarriot et al. 2015 \\
\hline
\end{tabular}


Increases validity of evaluation findings

Integrate multiple kinds of data (e.g., prior literature, stakeholder interviews, etc.)

Promotes stakeholder participation

Increases utilization of evaluation findings

Other

Inform future computational modeling

Well suited for multi-site evaluations

Inform longitudinal testing of program theory

Feedback loops were flexible for revision

Distinguish components of context, mechanism, and outcome for a realist evaluation

Identify where further evaluation is needed
Renmans et al. 2020

Fredericks et al. 2008

Fredericks et al. 2008

Biroscak et al. 2014; Alonge et al. 2017

Fredericks et al. 2008

Biroscak et al. 2014

Dyehouse et al. 2009

Renmans et al. 2020

The review of included studies identified strengths of causal-loop diagramming across five primary categories: understanding system components and behavior, clarifying the intervention, understanding implementation, communicating evaluation findings, and increasing the validity of evaluation findings. The most commonly mentioned strengths of using causal-loop diagrams was their ability to identify contextual elements (Renmans et al. 2020; Merrill et al. 2013; Rüegg et al. 2018; Mutale et al. 2017; Kwamie et al. 2014; Dyehouse et al. 2009) and to show the causal structure responsible for the behavior of outcome variables over time (Fredericks et al. 2008; Knai et al. 2018, Sarriot et al. 2015; Rüegg et al. 2018; Dyehouse et al. 2009). Clarifying the intervention through what-if scenarios (Okumu et al. 2016; Sarriot et al. 2015; Kwamie et al. 2014; Dyehouse et al. 2009) and identifying unintended consequences (Merrill et al. 2013; Fredericks et al. 2008; Mutale et al. 2017; Dyehouse et al. 2009) were also commonly 
mentioned. Several studies mentioned that causal-loop diagrams were useful for communicating complex issues (Renmans et al. 2020; Owen et al. 2018; Dyehouse et al. 2009).

Four categories of limitations were identified in the studies: necessary inputs, constraints of source data, limitations of the form of the diagrams, and communication limitations (Table 3). The time-intensive nature of causal-loop diagramming was the only limitation mentioned in studies across design approaches and is a constraint widely acknowledged in the system dynamics literature (Meadows 2008). Authors of four studies featuring secondary analysis of qualitative data (Knai et al. 2018, Owen et al. 2018, Okumu et al. 2016, Sarriot et al. 2015) described how their model development was constrained by the scope of the source data they used. The interviews used for these studies were conducted based on interview guides not designed to elicit information for causal-loop diagramming. The type of data useful for causal-loop diagramming (i.e., detailed descriptions of cause and effect relationships) differs to some degree from data routinely collected in qualitative interviews (i.e., narrative descriptions including implied communication). The resulting causal-loop diagrams, therefore, may inadvertently exclude variables and relationships existing in participants' mental models. 
Table 3. Limitations of causal-loop diagramming for program theory identified in included studies.

\begin{tabular}{|c|c|}
\hline Limitations of causal-loop diagramming & Source \\
\hline \multicolumn{2}{|l|}{ Necessary inputs } \\
\hline Time intensive & $\begin{array}{l}\text { Renmans et al. 2020; Merrill et al. } \\
\text { 2013; Dyehouse et al. } 2009\end{array}$ \\
\hline Resource intensive & Rüegg et al. 2018 \\
\hline Requires political and managerial buy-in & Rüegg et al. 2018 \\
\hline Requires leadership skills and a learning environment & Rüegg et al. 2018 \\
\hline $\begin{array}{l}\text { Requires comprehensive understanding of context and } \\
\text { program }\end{array}$ & Rüegg et al. 2018 \\
\hline Requires close analysis of data & Rüegg et al. 2018 \\
\hline \multicolumn{2}{|l|}{ Constrained by source data } \\
\hline Limited by scope of source data & $\begin{array}{l}\text { Knai et al. 2018; Owen et al. 2018; } \\
\text { Okumu et al. 2016; Sarriot et al. } \\
2015\end{array}$ \\
\hline Limited by the quality of source data & Renmans et al. 2020 \\
\hline \multicolumn{2}{|l|}{ Limitations of form } \\
\hline $\begin{array}{l}\text { Necessitated choosing between multiple possible } \\
\text { hypotheses }\end{array}$ & Renmans et al. 2020 \\
\hline $\begin{array}{l}\text { Does not include quantitative benchmarks for program } \\
\text { monitoring }\end{array}$ & Sarriot et al. 2015 \\
\hline Some micro-scale factors were black boxed & Sarriot et al. 2015 \\
\hline Diagram was limited by assumptions and simplifications & Alonge et al. 2017 \\
\hline $\begin{array}{l}\text { Does not account for other types of relationships (e.g., } \\
\text { linear, logarithmic, parabolic) }\end{array}$ & Renmans et al. 2020 \\
\hline $\begin{array}{l}\text { Less useful for identifying interventions than computational } \\
\text { modeling due to less precision }\end{array}$ & Biroscak et al. 2014 \\
\hline $\begin{array}{l}\text { Program theory might not be generalizable to other } \\
\text { programs }\end{array}$ & Biroscak et al. 2014 \\
\hline \multicolumn{2}{|l|}{ Communication limitations } \\
\hline $\begin{array}{l}\text { Diagrams with many variables and relationships can be } \\
\text { difficult to interpret }\end{array}$ & Renmans et al. 2020 \\
\hline
\end{tabular}

The form of causal-loop diagrams also constrained the type of information the diagrams could communicate (see Table 3, limitations of form). For example, quantitative benchmarks are not included in causal-loop diagrams. Diagrams with 
many variables and relationships can also be difficult to interpret, hindering communication.

\subsubsection{Centering the intervention vs. the problem}

Authors of the included studies also differed in how they situated the program or intervention in the context of the situation or problem in which it operates. Mutale and colleagues (2017), for example, described context as interacting with the intervention "in such a way as to modify, facilitate, or hinder the implementation of the intervention." Renmans and colleagues (2020), on the other hand, "stress the fact that an intervention is implemented in a pre-existing system/environment/context; the intervention influences the context, not the other way around." While both perspectives acknowledge the interaction and interdependence of a program and the situation in which it is embedded, the researchers illustrate a key difference in how diagrams describing program theory can either center the intervention itself or the problem (system) it is trying to change. ${ }^{2}$

\footnotetext{
${ }^{2} \mathrm{~A}$ system is defined as a set of interconnected elements or variables that are organized in a way that achieves a certain behavior or output (Meadows 2008). A problem is understood to be an undesirable configuration of a system that as described by behavior over time of key variables. These terms are used largely interchangeably in this text. Context is a term used in certain fields to describe the environment or setting in which variables of interest, such as an intervention, reside (Nilsen and Bernhardsson 2019).
} 


\subsection{Discussion}

\subsubsection{Summary of findings}

The 13 studies included in this review, described in 22 individual articles, represent applications of causal-loop diagramming to theory-based evaluation in a variety of fields, including health services, social marketing, economic development, and education. Authors used causal-loop diagramming as part of a systems approach to better understand interactions between context, intervention, and outcomes. Methods for developing these diagrams varied considerably: some studies engaged stakeholders or experts in an iterative modeling process; others derived diagram content through secondary analysis of prior evaluation data; and the remaining evaluators presumably developed their diagrams based on their mental models. Resulting diagrams varied in their sophistication and adherence to the best practices of system dynamics. Authors also differed in how they framed the relationship between context and intervention, and how they conceptualized their work in relation to program theory.

Strengths of using causal-loop diagrams identified by the study authors include better understanding system components and behavior, clarifying the intervention and implementation of it, communicating evaluation findings, and increasing the validity of evaluation findings. However, study authors also noted that this approach is time and resource intensive and the form constrains what 
can be included. Evaluators who based their diagrams on prior evaluation data that was not collected with causal-loop diagramming in mind felt constrained by the scope of the data available to them, indicating a need for strategies for collecting data suitable for modeling (see Chapter 4).

The studies identified in this review largely realized their goals to use causal-loop diagramming to explore the complex dynamics underlying programs, but the findings indicate the opportunity for further methods development and alignment with best practice of systems dynamics to more fully take advantage of the strengths of causal loop diagramming.

2.5.2. Comparing methods from included studies to system dynamics best practice

This review identified ways of theorizing programs and developing causal-loop diagrams differed in two key ways from system dynamics best practice. The difference in ways of conceptualizing the relationship between intervention and context identified in this review may reflect a fundamental difference in orientation between theory-based evaluation and system dynamics. Theorybased evaluation puts the program to be evaluated at the forefront and sees aspects of context as external influencers, as can be seen in the format of the pipeline logic model and other standard methods of diagramming program theory. System dynamics, on the other hand, centers the problem observed in the world by reproducing its key dynamics, and sees interventions as acting upon 
that pre-existing system. Reproducing the system configuration responsible for observed problem behavior enables system dynamicists to identify and evaluate possible points of leverage that could be exploited in the form of policies, programs, and other interventions. Basing the model on a coherent dynamic hypothesis-an explanation of how components of the system interact to produce observed behavior-provides a way to interpret program outcomes. The systems approach used in these studies, in which system boundaries are defined by an understanding of how problematic system behavior is produced, can be contrasted with a view of an intervention as situated in an exogenous and undefined context.

The process used in several studies (e.g., Dyehouse et al. 2009; Renmans et al. 2020), in which feedback loops are identified only after variables and relationships are included, reflects advice for causal-loop diagramming and systems mapping more broadly in the evaluation literature (Lee et al. 2016; Wright and Wallace 2020). Wright and Wallis (2020) even encourage the inclusion of as many variables and connections as possible when constructing causal maps, which goes against the 'as simple as possible but no simpler' norm in system dynamics. This practice of post-hoc identification of feedback loops does not reflect the standard process for developing causal-loop diagrams in mainstream system dynamics practice, in which model development is iterative and structure is guided by a dynamic articulation of system behavior (Sterman 2000). In other words, system dynamicists choose variables and feedback loops 
during model creation in part based on how the configuration of those loops describe the system behavior defined during problem articulation. Variables may be listed and relationships inventoried in model development, but this exercise takes place within a larger context of crafting a model that matches a modeler's "accumulated and abstracted understanding" (Eker and Zimmermann 2016) of system behavior. Relying solely on post-hoc identification of feedback loops, as was done in some of the included studies, risks preventing evaluators from taking full advantage of the strengths of causal-loop diagrams.

2.5.3. Aligning diagram development with system dynamics best practices The use of causal-loop diagrams for complexity-aware program theory may be better aligned with best practices in system dynamics by: 1) centering the problem, 2) matching model structure to system behavior, 3) using iterative, participatory methods to faithfully represent stakeholder mental models, and 4) including causal-loop diagramming early in program development.

\section{Center the problem}

System dynamics emphasizes modeling the problem or baseline situation to describe dynamics prior to intervention. Illustrating the dynamics of the problem allows for an assessment of how the problem is perpetuated, including implicit system goals and relevant aspects of context. Being precise about problem dynamics also provides an opportunity to identify appropriate interventions tailored to characteristics of the problem. 
Sterman (2000) describes modeling as an "inherently creative" but disciplined and rigorous process. The first step in the process-problem articulationinvolves defining the problem or behavior of interest guiding the modeling activity (see Figure 9 for an outline of Sterman's modeling process). In system dynamics, a problem is described through graphs of behavior over time for at least one key variable in a system. A graph of behavior over time shows how a variable increases or decreases over time, which provides clues about the underlying causal structure generating that behavior. Describing a problem in this way sets model boundaries, such as time horizon, general theme, and variables of interest. Setting boundaries distinguishes the model from the entire system, which provides crucial guidance for model building. 


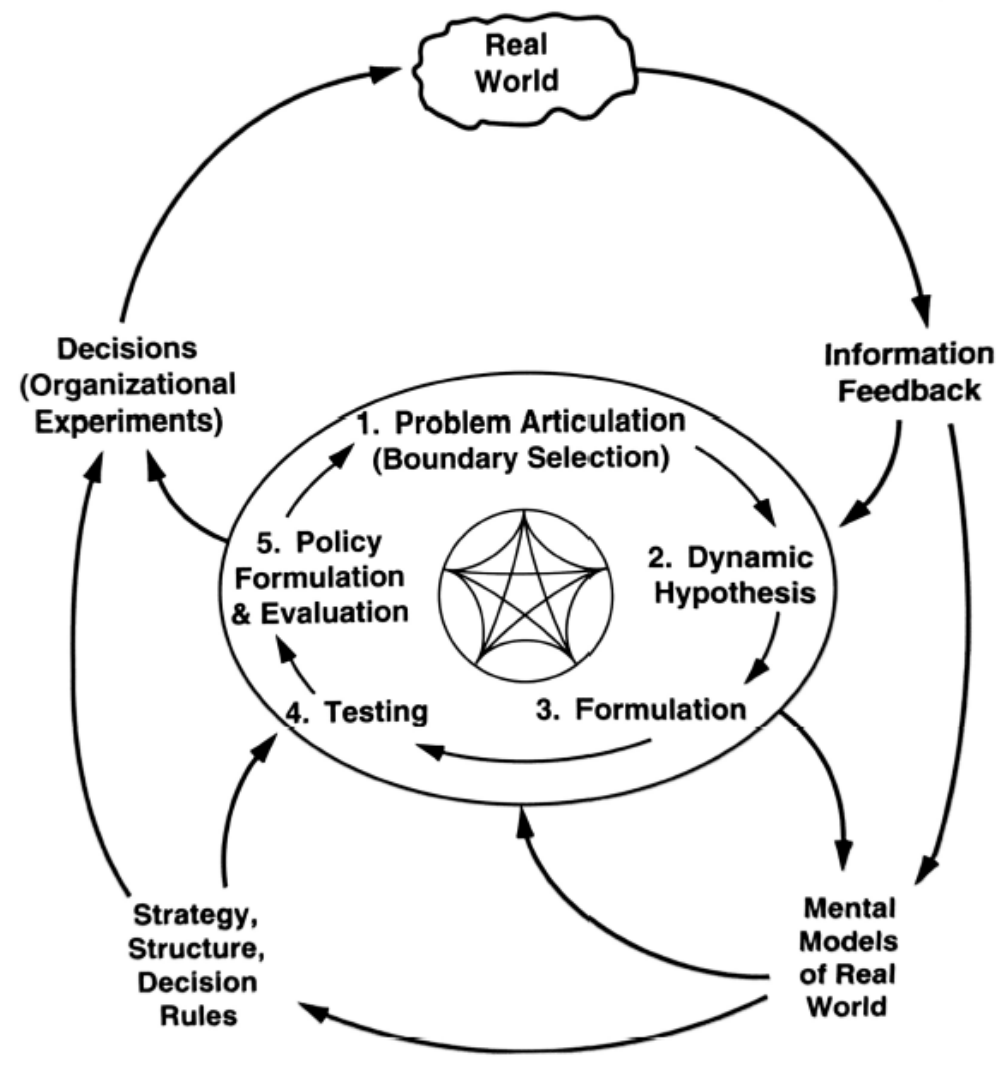

Figure 9. Iterative modeling process embedded in context. The numbered steps in the center circle are core steps in the modeling process. The lines between the steps at the center indicate iteration between steps. From Sterman 2000.

Sterman (2000) describes problem articulation as the most important step in the modeling process. "The art of model building is knowing what to cut out," Sterman (2000) writes, "and the purpose of the model acts as the logical knife. It provides the criteria to decide what can be ignored so that only the essential features necessary to fulfill the purpose are left."

When system dynamics modeling is done in the context of an organization, problem articulation is typically a participatory process involving model clients 
and/or stakeholders who know how the system works and can describe the problem. While the graphs themselves can be simple, the process of navigating multiple conflicting viewpoints among participants may not be. A significant body of knowledge in group model building contains strategies to engage groups in dialogue about identifying problem behavior (Vennix 1999; Hovmand 2012; Rouwette et al. 2002).

The second step in the modeling process is the formulation of a dynamic hypothesis-a theory describing why the problem exists. This hypothesis forms the basis for the causal structure of the model. As such, all variables important for describing how the problem behavior arose and is perpetuated should be incorporated into the model, even if they are seen as outside the direct influence of the program or organization.

By articulating the problem and formulating a dynamic hypothesis, the model building process is rooted in a particular mental model of the problem that determines boundaries useful for model building. Without grounding the model in an understanding of the problem in this way, causal mapping can become a sprawling exercise in including every possible variable and connection.

\section{Matching model structure to system behavior}

Causal-loop diagrams, and indeed all system dynamics models, are intended to model the key dynamics that produce system behavior. In computational system 
dynamics, models are validated by comparing graphs of behavior over time generated by the model with real-world data about the system of interest (also called reference behavior) (Sterman 2000). While the qualitative nature of causal-loop diagrams does not allow for such precise validation, modelers still try to match model structure to system behavior. For example, a system exhibiting exponential behavior would be dominated by reinforcing feedback (Anderson and Johnson 1997). Crafting models that plausibly reflect system behavior is a creative process that often involves multiple drafts and integration of multiple data sources (Sterman 2000).

Use iterative, participatory methods of diagram development to faithfully reflect stakeholder mental models

The use of iterative participatory approaches to generate causal-loop diagrams demonstrates an effort to align the diagram with stakeholder mental models. The studies utilizing a close analysis of prior evaluation data lack the iteration of the participatory approaches, so therefore less alignment can be assumed.

Nevertheless, the systematic use of qualitative data does demonstrate an attempt to center stakeholder mental models in the diagrams. In studies that did not describe methods for aligning the diagram to stakeholder mental models, one can assume that the diagram was created in a less rigorous fashion based on evaluators' understanding of the program and underlying system. To be successful, causal-loop diagrams should be clear about whose mental models are being represented. 
Studies that developed causal-loop diagrams based on prior evaluation data were limited by the scope of the data available to them, which was not designed with causal-loop diagramming in mind. Best practices in system dynamics involve iterative, participatory processes in which models are brought into alignment with stakeholder mental models.

Include causal-loop diagramming early in program development A key strength of system dynamics is its utility for identifying potential leverage points based on a sophisticated understanding of complex problem dynamics. Including causal-loop diagramming starting during initial needs assessment and program design and continuing through evaluation would leverage this strength.

\subsubsection{Limitations}

This review faced several constraints that may have limited the number of suitable studies found with the search strategy. Although gray literature was included to maximize the reach of the literature review, the scope of the review was limited to evaluation studies that were publicly available and found via an internet search. It is likely that causal-loop diagrams have been used in evaluations that were not identified in this review because they were not publicly available.

The imprecise terminology in this area may have also hindered the review. Many terms are used to refer to program theory, while it is also likely that there are 
program evaluations which used causal-loop diagramming in a way that would fit the inclusion criteria but used different terminology and were therefore excluded.

The review was also constrained by the level of detail included in the study articles about diagram development and use, and about the strengths and weaknesses encountered by teams during their diagramming efforts. Evaluators may have used more precise methods of diagram development than they had described, or excluded details about their experience using causal-loop diagramming because the primary focus of their publication was focused on content rather than methodology.

\subsubsection{Future research}

There is considerable opportunity to further assess the potential of causal-loop diagrams for program theory and build upon established best practice in systems science. Because documentation of model development was incomplete for some included studies, interviews with study authors could yield more information about best practices. Future studies could also formally evaluate the utility of causal-loop diagrams for program theory using qualitative methods to examine the experience of program staff and evaluators. Different diagramming methods could be applied to a common case to rigorously compare their information content and communicative value. Methods for generating causalloop diagrams of program theory in participatory but efficient ways suitable for the evaluation context could also be explored. Future research could also 
improve methods for gleaning causal-loop diagrams from existing qualitative data. The development of interview strategies for eliciting data suitable for analysis with methods like Kim and Andersen's (2012) could help align the scope of data collection to the purpose of causal-loop diagramming (see Chapter 4).

\subsection{Conclusion}

Standard methods of diagramming program theory do not incorporate complex aspects of context, change over time, and relationships between variables. This study identified and analyzed 13 studies utilizing causal-loop diagrams to aid theory-based evaluation through a systematic review of the literature. Included studies were developed through participatory methods, secondary analysis of prior evaluation data, or evaluator-led methods. Advantages of the causal-loop diagramming approach identified by study authors include understanding system components and behavior, clarifying the intervention or its implementation, communicating evaluation findings, and increasing the validity of findings. Limitations of the method include time and resource intensiveness, constraints of source data (for studies using secondary analysis), limitations of the form of causal-loop diagrams, and communication limitations. The use of causal-loop diagramming to enhance the development and utilization of program theory is promising and would be improved by closer integration with best practices from system dynamics: centering the problem, matching model structure to system behavior, using iterative, participatory methods of diagram development, and including causal-loop diagramming early in program development. 
3. Paper \#2: Reclaiming the 'loop' in causal-loop diagram: Advancing methods for identifying causal structures in qualitative data

Target journals: System Dynamics Review; Systems Research and Behavioral Science

\subsection{Abstract}

Existing methods for generating causal-loop diagrams from qualitative data have established initial processes for increasing transparency and rigor and demonstrated the potential of using system dynamics in qualitative analysis. These methods, however, are time consuming, rely exclusively on coding for individual causal links in model development, and do not adequately account for implicit communication or modeler influence. To address these limitations, this research presents a modified process for identifying causal structures (e.g., feedback loops) that utilizes software to make coding, tracking, and model rendering more efficient. This analysis process draws from existing methods, system dynamics best practice, and qualitative data analysis techniques. The use of resulting models for qualitative research and system dynamics modeling is discussed.

\subsection{Introduction}

Qualitative research, particularly interviewing, has long been used in the development of system dynamics models, although the exact methods for gleaning model data from qualitative data have not always been specified (Eker and Zimmermann 2016; Luna-Reyes and Andersen 2003). Recently, more 
attention has been paid within the system dynamics field to adding methodological rigor to the process of building diagrams and models from qualitative data (Kim and Andersen 2012; Turner et al. 2013; Yearworth and White 2013; Biroscak et al. 2014). Such rigor enhances the credibility of system dynamics models and opens the door to broader uses in applications such as qualitative research and program evaluation.

Kim and Andersen (2012) provide a detailed description of one method that borrows from grounded theory methodology. Their approach—which has been termed purposive text analysis-involves open coding to identify themes and individual causal relationships, visualizing these relationships as causal segments, diagram editing, and creation of an evidence table. These steps enable tracking of specific causal claims in the model. Distinguishing between information provided by sources and assumptions made by the modeler introduces transparency into the interpretive aspects of model building. As Kim and Andersen note in their discussion, producing maps using this kind of predetermined process "shift[s] power from the modeler to the data" (ibid). Modeling is still an interpretive process, but the modeler's subjective influence is tracked and made transparent for the end users of the model, enhancing credibility and reproducibility.

However, this specificity comes at the cost of substantial time and effort on the part of the modeler, as acknowledged by the authors. Several subsequent 
studies have attempted to streamline this method to be less labor intensive while retaining transparency and systematic generation of causal maps (Eker and Zimmermann 2016; Turner et al. 2014; Turner, Kim, and Andersen 2013; Biroscak et al. 2014). Eker and Zimmerman (2016) adapted Kim and Andersen's method by introducing causal connections in the post-coding analysis phase, rather than the coding phase. In their formulation, qualitative data is coded according to standard thematic procedures, then code groups are developed using axial coding. Relationships between code groups are then identified. The resulting causal map describes high-level dynamics. While this method is less time-consuming than Kim and Andersen's method, it introduces more subjectivity into the modeling process, as relationships are identified during analysis, rather than directly identified in source data.

Turner and colleagues (2013) adjusted the coding procedure to fit asynchronous meetings with three distinct stakeholder groups. In a subsequent study, Turner and colleagues (2014) compare the coding methods used in this article with the original method published by Kim and Andersen (2012) and identify six dimensions of research design relevant to studies using text-derived causal mapping: synchronous versus asynchronous communication of participants, one versus many groups, context set by researchers versus by participants, data collected by researcher versus not collected by researcher, one versus many coders, and coders engaged versus not engaged in data collection. The 
researchers provide guidance regarding how these design choices affect the design process in text-derived causal mapping.

Biroscak and colleagues (2014) adapted Kim and Andersen's method to diagram a theory of change for a community-based social marketing program. In an iterative fashion, the researchers coded and analyzed transcripts from various program meetings, training sessions, and interviews and then used that content analysis as the foundation for group model-building sessions. Participants in the model-building sessions provided input into model purpose and structure.

Yearworth and White (2013) also use modeling to enhance the coding phase of qualitative research. Using NVivo computer-assisted qualitative data analysis software (CAQDAS), the authors create tables based on code co-occurrence and generate causal-loop diagrams based on these tables. While these diagrams can be completed quickly and without an additional subjective modeling step, it is unclear whether co-occurrence truly predicts causation.

To reduce the documentation burden of Kim and Andersen's method (2012), Eker and Zimmerman (2016) identify hierarchical relationships between generalized variables found in the text in a manner similar to thematic analysis. Causal relationships between those variables are then identified by the modeler. A similar approach has been used to generate fuzzy cognitive maps from qualitative data (Alibage et al. 2018; Alizadeh and Jetter 2017). The use of 
generalized variables does make analysis less time consuming than coding individual causal relationships, but it introduces an additional type of abstraction undertaken by the modeler. This approach may be most appropriate for summarizing the perspectives of a group of participants whose mental models are similar and when precise tracking of causal statements is less important.

Kim and Andersen's method (2012) and its adaptations provide guidance about how to generate maps from causal structures once the causal structures have been identified, but do not provide much advice about identifying the causal structures in the first place. Moreover, their focus on individual causal links precludes attention to other components of causal-loop diagrams, such as feedback loops and boundaries. Well-constructed causal-loop diagrams are more than compilations of individual connections; they describe the key dynamics of a system, as manifested in a person's mental model.

According to Sterman (2000), causal-loop diagrams—and system dynamic models generally—should in the end reflect a coherent dynamic hypothesis about how system structure produces observed behavior. A configuration of feedback loops is carefully chosen during the model development process, not merely observed post hoc from an accumulation of variables and relationships. It is true that individual mental models are likely not as tidy and coherent as wellcrafted system dynamics models, and therefore causal-loop diagrams used to represent mental models found in qualitative data should not be judged by the 
same standards. However, methods for gleaning causal-loop diagrams from qualitative data may be strengthened by a greater focus on causal structures larger than individual links, such as feedback loops.

Another reason for developing methods for identifying larger causal structures in qualitative data stems from how we communicate. Verbal communication involves a fair amount of implied information (Grice 1975), which might be missed if coding only takes place at the level of individual links. So methods that identify feedback loops post hoc may miss causal structures that were implied but not explicitly outlined by the participant.

Prior methods have also not kept track of whether a model component is explicitly mentioned in the interview data, implied by the interviewee, or imputed during the process of modeling. Variables or relationships introduced by the modeler can carry with them the assumptions of the modeler, which may or may not be shared by the participant. Capturing this information and making it available would enhance the transparency of models generated from interview data and would enable researchers to identify information gaps. Methods for tracking modeler hypotheses during analysis may also be helpful.

This body of work by Kim and Andersen (2012) and subsequent studies illustrate a need for methods for generating system dynamic models from qualitative data in a way that is time efficient, faithful to source data, and accounting of modeler 
input. But these methods do not go far enough in capturing larger (often implied) causal structures and tracking source data. In this paper, I build on this prior work to outline a proposed method for generating causal-loop diagrams from qualitative data that addresses these challenges. The method, termed here causal structure mapping, aims to reliably represent the mental models embedded in participant narratives in the form of causal-loop diagrams by identifying causal structures through close analysis of qualitative data. Practices in qualitative research and system dynamics modeling inform guidance for coding and model formation. The use of software further streamlines these tasks. This research exists in the larger context of efforts to generate complex systems diagrams and models systematically from qualitative data (Alibage 2020; Alizadeh and Jetter 2017; Abdelbari and Shafi 2017; Sonawane et al. 2014).

\subsection{Study setting}

Qualitative interview data from an ongoing implementation science study was used to illustrate the proposed analysis process. The data set included semistructured qualitative interviews with six practice facilitators working to improve screening, brief intervention, and referral to treatment (SBIRT) for unhealthy alcohol use in primary care clinics in Oregon. Practice facilitators are skilled individuals who provide support for the adoption of evidence-based practices within primary care (Baskerville et al. 2012). The longitudinal study is being conducted by the Oregon Rural Practice-based Research Network (ORPRN), housed at Oregon Health and Science University. The aim of the baseline 
interviews analyzed for this research was to better understand how practice facilitators tailor implementation support based on clinic differences, personal expertise, and characteristics of the evidence-based clinical intervention. To address the question of tailoring, practice facilitators' mental models of clinical practice change were examined. The same analyst [ESK] who conducted and qualitatively analyzed the interviews subsequently conducted the causal-loop diagram mapping analysis. Diagrams produced in this analysis will be compared with those produced in future rounds of data collection as part of a longitudinal study.

\subsection{Recognizing causal structures}

In order to code for causal structures in qualitative data, one must be able to recognize them. Causal-loop diagrams contain a variety of causal structures at different scales, including individual variables, causal links, feedback loops, and archetypes. As seen in Figure 10, these structures are hierarchically related, with increasing causal information contained in structures with increasing complexity. 


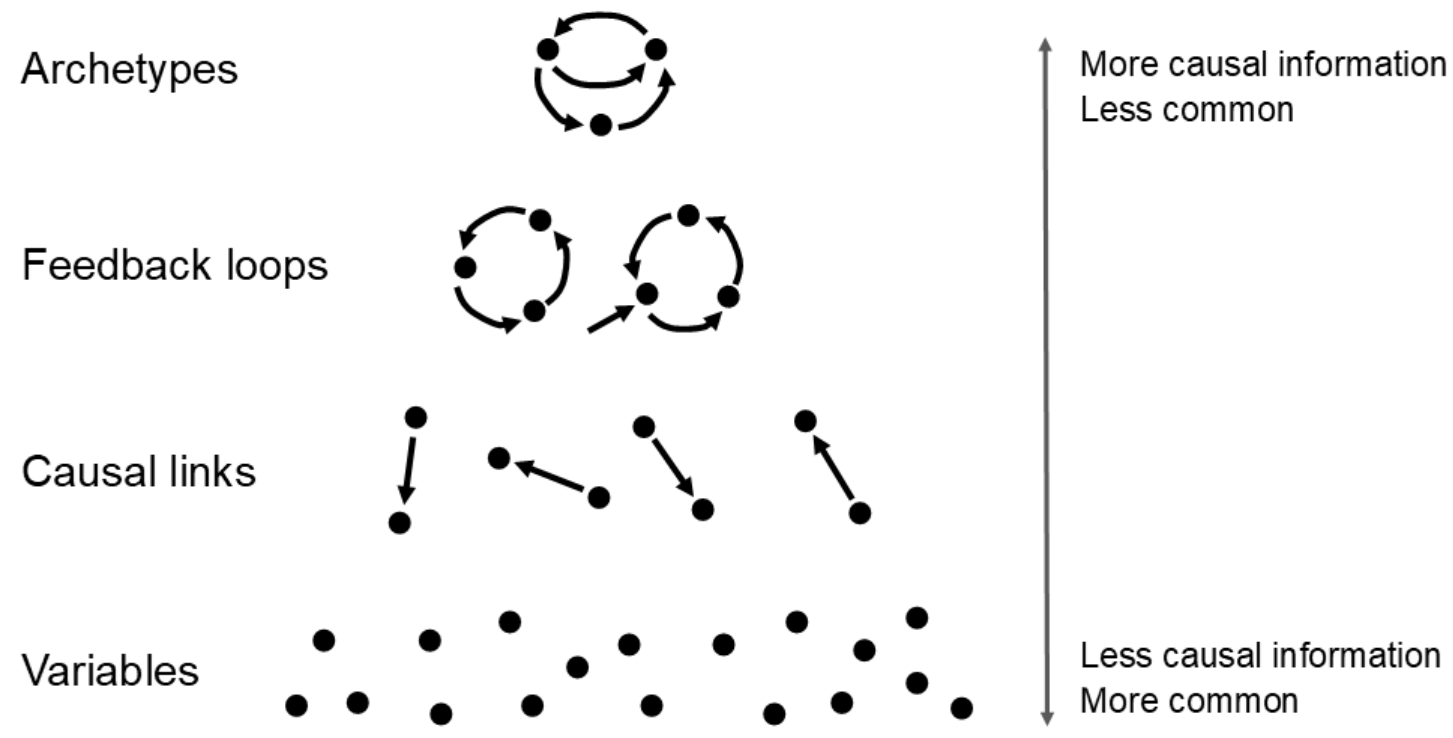

Figure 10. Hierarchical relationships between variables, causal links, feedback loops, and archetypes. Variables are elements in a system that can be isolated or connected and that show a pattern of behavior over time. Causal links are unidirectional relationships describing cause and effect. Feedback loops can be reinforcing or balancing and consist of circular causal connections. Archetypes are certain configurations of loops and variables describing common system structures that produce predictable behavior. Model components contain more causal information and become less common higher in the hierarchy. See Sterman (2000) for further description of basic causal structures and Senge (2010) for further description of archetypes.

In system dynamics, anything that has the capacity to increase or decrease over time can be considered a variable. This categorization includes tangible quantities of things that exist in the world, such as water (as in the well-known bathtub examples for system dynamics), people, and resources; internal mental states, such as happiness or confidence; or other abstract quantities, such as the likelihood of an event. The best practice for labeling variables in system dynamics is to do so in a way that indicates presence of the quantity, unless doing so interferes with comprehension (Sterman 2000; Anderson and Johnson 1997). For example, clinic bandwidth is better phrasing than lack of time or clinic busy with other things. 
The choice of variables to include in a model is determined by the problem or system behavior the modeler is trying to better understand (Sterman 2000). The "story" of a problem in a system dynamics model is told by describing how key system variables change over time. Key system variables for a predator-prey system, for example, would be populations of predator and prey species. Having the problem determine the variables included in the model provides crucial guidance about system boundaries.

Variables in system dynamics are considered to be endogenous to the system if they are determined by other variables in the model (Sterman 2000). Clinic bandwidth, for example, could be a function of factors like visit volume, patient complexity, efficiency of workflows, and the skills of clinicians and staff. Exogenous variables_also called drivers_influence endogenous variables, but are not themselves affected by any other variables in the model (Ford 2010; see Figure 11). Because exogenous variables are assumed to be constant, they serve as a type of model boundary. The choice to consider a variable exogenous is made when the factors influencing that variable are not important to describing how the endogenous system variables change over time. For example, community resources or statewide policies might be considered exogenous variables in a model describing clinic dynamics if their influence could be considered constant in the model. 


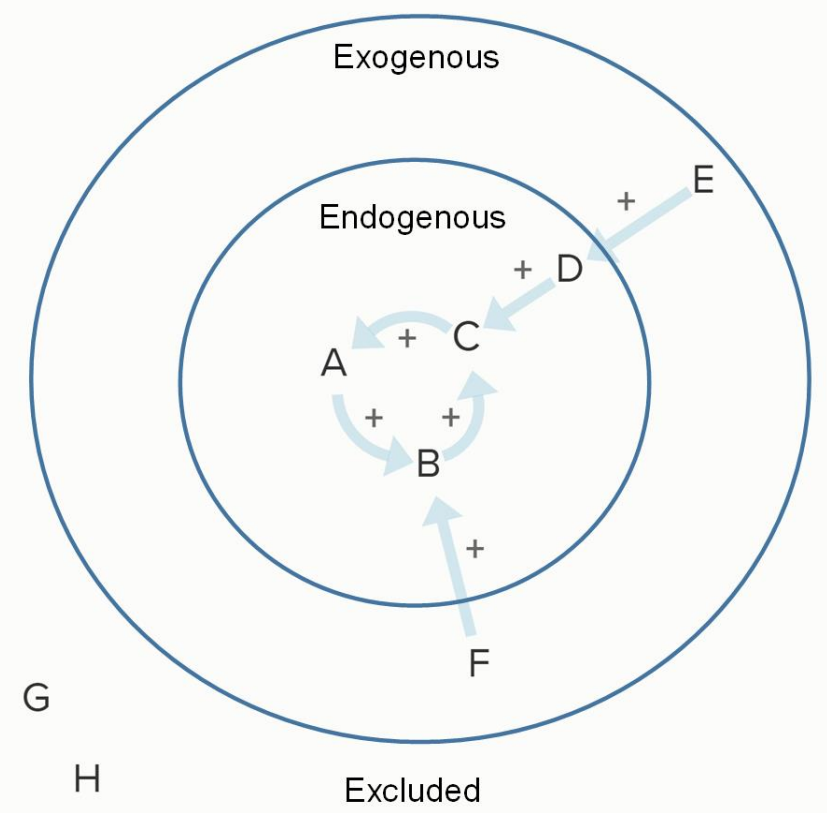

Figure 11. Types of system boundaries for causal-loop diagramming. In the center of the diagram, variables $A, B$, and $C$ exist in a reinforcing feedback loop. Variables $A, B, C$, and $D$ can be considered endogenous because their behavior is determined by other variables in the model. Variables $\mathrm{E}$ and $\mathrm{F}$ are exogenous drivers to the system because they affect it, but are not themselves determined by variables in the model. Variables $\mathrm{G}$ and $\mathrm{H}$ are excluded; their existence is acknowledged but they are not connected with other variables in the model. Distinguishing endogenous, exogenous, and excluded variables constitutes boundary selection in system dynamics modeling. Adapted from Ford (2010).

Feedback loops are a defining characteristic of causal-loop diagrams (Sterman 2000; Meadows 2008; Anderson and Johnson 1997). In system dynamics models and in the complex systems they represent, feedback relationships are the source of nonlinear behavior. Feedback loops reflect commonly understood dynamics, but can themselves be difficult to recognize. Reinforcing feedback loops - in which effects are compounded and growth or decline is exponentialare often described as 'vicious' or 'virtuous' cycles (Meadows). Reinforcing behavior is dominant when a system is being pulled out of balance or getting 'out of control.' A balancing feedback loop, in which change in one direction is 
countered by change in the opposite direction, brings a system toward an implicit or explicit goal or set point (Sterman 2000).

In natural language, a person's description of how they pursued a goal can contain a significant amount of implicit information. For example, it is reasonable to assume that the mental model of somebody who says they are trying to lose weight or learning to play the piano likely includes the variables outlined in Figure 12-desired and actual states, a gap describing the difference between them, and actions taken for improvement. However, speakers do not necessarily identify each of these distinct variables and the causal relationships between them, presumably because a shorthand phrase is sufficient for communicating the basic idea of goal-directed behavior. The phrase vicious cycle mentioned above similarly conveys information about causal structure without explicitly outlining the variables in a reinforcing loop.

A.

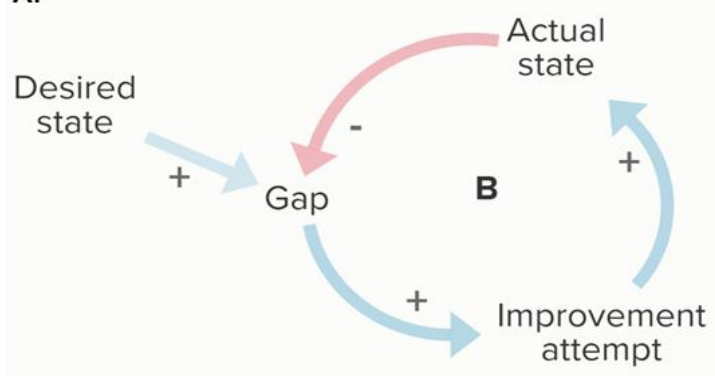

B.

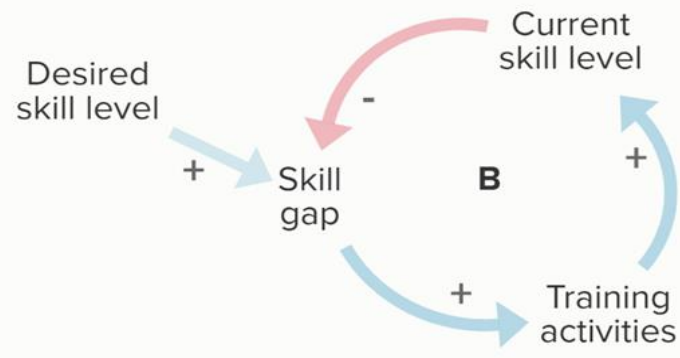

Figure 12. Generic structure and example of goal-directed balancing feedback loops. Causal-loop diagrams of a generic structure $(A)$ and an example (B) show the structure of goaldirected feedback loops. In Figure 12A, a gap variable describes the difference between the actual state or level and the desired state. The larger this gap, the larger the improvement attempt that is made to try to bring the actual in line with the desired. As improvement attempts increase, the actual state is improved and the gap is decreased. Over time, the actual state 
trends toward the desired state, all else being equal. Figure 12B describes the same structure using an example of pursuing training in order to improve skills to a desired level. Plus signs in causal-loop diagrams indicate a causal relationship in the same direction, while negative signs indicate opposite causal effects. The letter "B" is included inside the feedback loops to indicate a balancing feedback loop.

Archetypes are certain configurations of variables and loops that have been recognized by the systems science community as describing a particular system behavior common across multiple settings (Kim 1994; Kim and Anderson 2007; Senge 2010; Meadows 2008). A popular example is the tragedy of the commons, in which a shared resource is exploited and ultimately eliminated due to a shortsighted incentive structure. The phrase arms race communicates the escalation archetype, in which competing actors devote increasing amounts of resources to best one another. As with feedback loops, phrases such as tragedy of the commons or arms race convey a significant amount of implicit information about causal structure. If analyzed using methods that detect only explicit causal links, information about these larger causal structures would be missed.

Moreover, causal structures such as feedback loops and archetypes can exist in a person's mental model, and be evident in their description of it, without that person being aware of those dynamics or using certain phrases. A skilled modeler can recognize and inquire about these causal structures, as is commonly done in facilitation for group model building (Hovmand et al. 2012). A key aim of the current research is to adapt existing methods for generating causal-loop diagrams from qualitative data to account for implicit communication of causal structures. 


\subsection{Analysis process}

The process outlined here has been designed to improve upon prior methods of purposive text analysis to increase time efficiency, tracking of contributions, and orientation toward larger causal structures. Analysis steps are informed by a blend of qualitative research methods, prior mapping analysis methods, and standards and norms for creating causal-loop diagrams from system dynamics (Braun and Clarke 2006; Sterman 2000; Kim and Andersen 2012). The nine steps in the analysis process are outlined in Table 4.

Table 4. Analysis process for generating causal map from qualitative data

\begin{tabular}{|l|l|l|l|l|}
\hline \multicolumn{1}{|c|}{ Analysis step } & \multicolumn{1}{|c|}{ Approach } & Source of approach & \multicolumn{1}{|c|}{ Input } & \multicolumn{1}{|c|}{ Output } \\
\hline $\begin{array}{l}\text { 1. Get familiar } \\
\text { with data }\end{array}$ & $\begin{array}{l}\text { Read transcript, } \\
\text { listen to audio } \\
\text { recording }\end{array}$ & $\begin{array}{l}\text { Qualitative analysis } \\
\text { (e.g., Braun and } \\
\text { Clarke 2006) }\end{array}$ & $\begin{array}{l}\text { Interview } \\
\text { transcripts and } \\
\text { audio } \\
\text { recordings }\end{array}$ & $\begin{array}{l}\text { Big-picture } \\
\text { understanding of } \\
\text { data }\end{array}$ \\
\hline $\begin{array}{l}\text { 2. Review } \\
\text { research } \\
\text { questions / focus }\end{array}$ & $\begin{array}{l}\text { Identify relevant } \\
\text { mental model(s) } \\
\text { and associated } \\
\text { boundaries }\end{array}$ & $\begin{array}{l}\text { Qualitative analysis, } \\
\text { systems dynamics }\end{array}$ & $\begin{array}{l}\text { Research } \\
\text { questions, } \\
\text { research } \\
\text { proposal }\end{array}$ & $\begin{array}{l}\text { Orientation toward } \\
\text { needed } \\
\text { information }\end{array}$ \\
\hline $\begin{array}{l}\text { 3. Identify, code, } \\
\text { and make note of } \\
\text { casual structures }\end{array}$ & $\begin{array}{l}\text { Code causal } \\
\text { structures and } \\
\text { summarize in } \\
\text { causal-loop } \\
\text { diagram notation }\end{array}$ & $\begin{array}{l}\text { Qualitative analysis; } \\
\text { Kim and Andersen } \\
\text { (2012); system } \\
\text { dynamics }\end{array}$ & $\begin{array}{l}\text { Qualitatively } \\
\text { coded } \\
\text { quotations }\end{array}$ & $\begin{array}{l}\text { Causal structures } \\
\text { identified with } \\
\text { codes unique to } \\
\text { specific claim; } \\
\text { unique IDs } \\
\text { attached }\end{array}$ \\
\hline $\begin{array}{l}\text { 4. Generate } \\
\text { query report with } \\
\text { coded data }\end{array}$ & $\begin{array}{l}\text { Use CAQDAS to } \\
\text { generate report }\end{array}$ & Qualitative analysis & $\begin{array}{l}\text { Coded } \\
\text { documents }\end{array}$ & $\begin{array}{l}\text { Query report } \\
\text { including } \\
\text { quotations, codes, } \\
\text { comments with } \\
\text { causal structures, } \\
\text { and quotation } \\
\text { numbers }\end{array}$ \\
\hline $\begin{array}{l}\text { 5. Sketch causal- } \\
\text { loop diagrams of } \\
\text { loops and } \\
\text { archetypes }\end{array}$ & $\begin{array}{l}\text { Freehand draw } \\
\text { using causal- } \\
\text { loop diagram } \\
\text { notation }\end{array}$ & System dynamics & $\begin{array}{l}\text { Query reports } \\
\text { aketches of loops } \\
\text { with quotation } \\
\text { numbers attached }\end{array}$ \\
\hline
\end{tabular}




\begin{tabular}{|l|l|l|l|l|}
\hline $\begin{array}{l}\text { 6. Create \& clean } \\
\text { up causal } \\
\text { mapping table }\end{array}$ & $\begin{array}{l}\text { Aggregation of } \\
\text { causal links into } \\
\text { table }\end{array}$ & $\begin{array}{l}\text { Kim and Anderson } \\
\text { (2012); requirements } \\
\text { of visualization } \\
\text { platform }\end{array}$ & $\begin{array}{l}\text { Query reports, } \\
\text { causal-loop } \\
\text { diagram } \\
\text { sketches }\end{array}$ & $\begin{array}{l}\text { Table detailing } \\
\text { variables, links, } \\
\text { direction, valence, } \\
\text { tags, descriptions, } \\
\text { and quotation } \\
\text { numbers }\end{array}$ \\
\hline $\begin{array}{l}\text { 7. Render } \\
\text { causal-loop } \\
\text { diagrams using } \\
\text { visualization } \\
\text { software }\end{array}$ & $\begin{array}{l}\text { Upload table; } \\
\text { rearrange } \\
\text { according to } \\
\text { causal-loop } \\
\text { diagram norms }\end{array}$ & $\begin{array}{l}\text { System dynamics; } \\
\text { procedures of } \\
\text { visualization software }\end{array}$ & $\begin{array}{l}\text { Causal } \\
\text { mapping table; } \\
\text { visualization } \\
\text { software }\end{array}$ & $\begin{array}{l}\text { causal-loop } \\
\text { diagrams rendered } \\
\text { in digital } \\
\text { visualization } \\
\text { platform }\end{array}$ \\
\hline $\begin{array}{l}\text { 8. Refine causal- } \\
\text { loop diagrams }\end{array}$ & $\begin{array}{l}\text { Edit model to } \\
\text { reduce repetition } \\
\text { \& for logical } \\
\text { clarity }\end{array}$ & $\begin{array}{l}\text { Criteria described in } \\
\text { current paper } \\
\text { informed by system } \\
\text { dynamics }\end{array}$ & $\begin{array}{l}\text { Rendered } \\
\text { causal-loop } \\
\text { diagrams in } \\
\text { visualization } \\
\text { software }\end{array}$ & $\begin{array}{l}\text { Revised causal- } \\
\text { loop diagrams in } \\
\text { visualization } \\
\text { software }\end{array}$ \\
\hline $\begin{array}{l}\text { 9. Analyze } \\
\text { mental model(s) } \\
\text { using causal- } \\
\text { loop diagrams }\end{array}$ & $\begin{array}{l}\text { Modeler review } \\
\text { of causal-loop } \\
\text { diagrams }\end{array}$ & $\begin{array}{l}\text { Research questions, } \\
\text { guidance outlined in } \\
\text { current paper } \\
\text { informed by system } \\
\text { dynamics }\end{array}$ & $\begin{array}{l}\text { Revised } \\
\text { causal-loop } \\
\text { diagrams }\end{array}$ & \begin{tabular}{l}
$\begin{array}{l}\text { Narrative and } \\
\text { diagram } \\
\text { descriptions of } \\
\text { gaps in causal } \\
\text { models, } \\
\text { comparisons } \\
\text { between } \\
\text { diagrams, etc. }\end{array}$ \\
\hline
\end{tabular}
\end{tabular}

To streamline the coding and model generation process, two types of software are used. ATLAS.ti (Version 8.0, Scientific Software Development GmbH), a CAQDAS program, is used to keep track of causal structures associated with source text. Kumu, a web-based data visualization platform created initially for network modeling, ${ }^{3}$ is used to render the causal-loop diagram from data about those structures. The use of these software tools is intended to facilitate easier and more robust tracking of source material and modeler input, and to allow greater modeler engagement with qualitative source material when identifying key model dynamics.

${ }^{3}$ www.kumu.io 
In line with qualitative methods of thematic analysis, the first step (step 1 in Table 4) was to get familiar with the data through listening to audio recordings and reviewing transcripts (Braun and Clarke 2006). This informal phase oriented the analyst to the data and allowed a "big picture" understanding to start to develop.

Research questions were reviewed to orient the analyst toward needed information (step 2). Transcripts were entered into ATLAS.ti, coded for causal information (step 3), and then query reports were generated compiling coded interview segments (step 4). Query reports were reviewed and larger causal structures (feedback loops and archetypes) are sketched using close reading of the source text (step 5). A table compiling causal and attribution data was produced (step 6) and uploaded for visualization (step 7). The causal-loop diagrams were refined (step 8) and analyzed (step 9). Because the research question for the ORPRN study involved comparing practice facilitator mental models, separate causal-loop diagrams were created for each participant. The following sections provide further detail about steps 3-9.

\subsubsection{Coding for causal structures (step 3)}

Because this causal mapping was done as a secondary analysis, the data used for this study were already uploaded to a common file in ATLAS.ti and coded and analyzed using thematic analysis as outlined by Braun and Clarke (2006). Therefore, the data had been segmented into quotations with associated codes and automatically numbered by the software according to the document number and the order of the quotation. For example, the second quotation in document 4 
was numbered 4:2. Each quotation contained a portion of the interview in which a single idea or set of ideas were described. Had the data not been previously coded, segmentation could have been done during this step. Codes corresponding to the components of causal maps outlined in the previous section, which are outlined in Table 5, were applied to the existing quotations in ATLAS.ti.

Table 5. Codes indicating model components used during analysis.

\begin{tabular}{|c|c|}
\hline Code & Definition \\
\hline Causal_archetypes & $\begin{array}{l}\text { Explicit or implied references to system archetypes or common } \\
\text { structures }\end{array}$ \\
\hline Causal_behavior & $\begin{array}{l}\text { Descriptions of how system or variable behavior change over time, } \\
\text { particularly pertaining to problem definition }\end{array}$ \\
\hline Causal_boundaries & $\begin{array}{l}\text { References to what is included vs. excluded, important vs. less } \\
\text { important, inside vs. outside scope, etc., to understanding the problem } \\
\text { behavior }\end{array}$ \\
\hline $\begin{array}{l}\text { Causal_feedback } \\
\text { loops }\end{array}$ & Explicit or implicit references to reinforcing or balancing feedback loops \\
\hline Causal_link & Explicit or implicit references to causal relationships between variables \\
\hline Causal_variable & $\begin{array}{l}\text { References to variables or factors relevant to understanding the problem } \\
\text { behavior. This code is used for isolated variables that are not mentioned } \\
\text { in the context of a causal link, feedback loop, or archetype. }\end{array}$ \\
\hline
\end{tabular}

Data were coded using structures that were as large as possible, in order to preserve the key dynamics of the data. For example, when a feedback loop was observed, it was coded as such, even though it could have been coded as a series of individual causal links. 


\title{
During the coding process, variables and causal structures were described in
}

\section{quotation comments using a combination of causal-loop diagram notation and}

\author{
narrative text (see Table 6 for examples).
}

Table 6. Coding examples from ORPRN study.

\begin{tabular}{|c|c|c|}
\hline Quotation & Code & Comment \\
\hline $\begin{array}{l}\text { I think with a little bit of empowerment you can kind } \\
\text { of build a champion, even if somebody doesn't } \\
\text { come forward as "I am the champion", then it's still } \\
\text { possible to maybe through some motivational } \\
\text { interviewing, like elicit some motivation and kind of } \\
\text { collaboratively design a champion. (Participant 3) }\end{array}$ & Causal_links & $\begin{array}{l}\text { Motivational interviewing --> } \\
\text { empowerment --> champion }\end{array}$ \\
\hline $\begin{array}{l}\text { I think that training that I've received since l've } \\
\text { started ORPRN is going to be really valuable also. } \\
\ldots . . \text { I'm really ... . a doer. I learn by doing things } \\
\text { and without context for the things that I'm learning, } \\
\text { I can sometimes struggle to apply that knowledge. } \\
\text { I'm both eager and nervous to get out there and } \\
\text { start applying the knowledge that I've gained ... } \\
\text { because that's really how I think I'm going to get } \\
\text { the most out of what I've had the opportunity to } \\
\text { learn and hope to learn that a bit better. } \\
\text { (Participant 5) }\end{array}$ & $\begin{array}{l}\text { Causal_loop } \\
\text { s }\end{array}$ & $\begin{array}{l}\text { PERC }{ }^{1} \text { training --> PERC } \\
\text { knowledge and skill --> } \\
\text { PERC application of } \\
\text { knowledge with clinics --> } \\
\text { PERC knowledge and skill } \\
\text { (reinforcing) }\end{array}$ \\
\hline $\begin{array}{l}\text { [The clinic] had a very specific EMR-related } \\
\text { request [the fulfillment of which] would make [their] } \\
\text { reporting way easier... They were already } \\
\text { planning to report for that metric and hoping to } \\
\text { meet, they call it the cutoff, the baseline, the } \\
\text { benchmark. ... Their concerns had to do with IT } \\
\text { constraints but they... had a sense for what their } \\
\text { numbers were and felt that what they were doing } \\
\text { met the criteria as far as screening and the } \\
\text { intervention. (Participant } 4 \text { ) }\end{array}$ & Causal_links & $\begin{array}{l}\text { EHR / IT constraints --> (-) } \\
\text { clinic ability to report on } \\
\text { SBIRT }\end{array}$ \\
\hline $\begin{array}{l}\text { I always mention that I'm based in [small town] and } \\
\text { then I'm from [rural area]. That's very intentional. } \\
\text { So, I feel like it's someone who gets it, gets the } \\
\text { area, because sometimes it makes a difference if } \\
\text { they think it's someone from [urban center] coming } \\
\text { in and maybe not having any idea of the area or } \\
\text { [inaudible] that I think people are more comfortable } \\
\text { with. ... [I make an effort to draw] those little } \\
\text { connections in that way while still showing I'm } \\
\text { familiar with the area. I'm totally comfortable driving } \\
\text { up there now in the winter, whatever. So, I think } \\
\text { [it's] both [to build rapport and show familiarity with } \\
\text { context], honestly. (Participant 2) }\end{array}$ & Causal_links & $\begin{array}{l}\text { PERC regional affiliation --> } \\
\text { (PERC-C/S relationship) }\end{array}$ \\
\hline
\end{tabular}

${ }^{1} \mathrm{PERC}=$ practice enhancement research coordinator, a practice facilitator role at ORPRN 
Individual variables are ubiquitous in qualitative data. In system dynamics modeling, variables are nouns that could increase or decrease in some way (e.g., quantity) and are phrased in a way that indicates presence (Sterman 2000). Capturing variables that fit these criteria in source text required a degree of translation between interviewees' natural language and causal-loop diagram notation (see Table 6 for coding examples).

When coding for causal links, multiple types of statements were identified, such as if/then statements, hypotheticals, and counterfactuals. Implied variables were noted in parentheses. Causal segments were not created for every statement in the interview. Choices for what to code were guided by the research questions and what informants focused on in their interview.

Code descriptions for feedback loops contained a combination of casual links and narrative description (see Table 6). Reinforcing feedback loops were indicated by descriptions of mutually amplifying variables, exponential behavior, or terms such as "vicious" or "virtuous" cycle. Balancing feedback loops were often indicated by mention of implicit or explicit goals and actions made to achieve them. Enough description was provided in the coding notes to enable later sketching of those causal structures, but the notes for larger causal structures did not necessarily include every variable and relationship. Positive (-$>$ ) or negative $(-->(-))$ valence of causal connections was indicated. 
Descriptions of behavior over time or instances in which effect variables caused further change to their causes were indications of a feedback relationship. The "causal_behavior" code was used in two ways: when a participant identified a variable as being an indicator of system performance, or when they described the behavior of that variable over time.

Many quotations included multiple types of causal structures. For these quotations, the appropriate causal codes from Table 5 were applied and the corresponding comments were divided according to code. For example, loops and links were listed separately within one comment. The quotation numbers tied to sections of text generated by ATLAS.ti were used as identification tags to trace variables and causal links to places in the text. The notation describing causal structures used in the quotation comments illustrated in Table 6 adhered to standard norms for causal-loop diagramming (Sterman 2000; Anderson and Johnson 1997), which are summarized in Table 7.

Table 7. Design features of causal-loop diagrams

\begin{tabular}{|l|l|}
\hline Diagram feature & Description \\
\hline Variable names & $\begin{array}{l}\text { Indicates presence of a countable noun (e.g., Trust between facilitator } \\
\text { and staff; Clinic knowledge of quality improvement; Motivation to provide } \\
\text { better care) }\end{array}$ \\
\hline Arrow directionality & Unidirectional \\
\hline Arrow valence & $\begin{array}{l}\text { Positive or negative valence. The form of the link must equate to an } \\
\text { increase in A results in an increase or decrease in B. }\end{array}$ \\
\hline Visual layout & $\begin{array}{l}\text { Minimize overlap; make loops explicit; cluster variables with similar } \\
\text { themes when possible }\end{array}$ \\
\hline Endogenous vs. & Endogenous variables connected toward center of diagram; exogenous \\
\hline
\end{tabular}


3.5.2. Generation of query reports and sketching causal structures (steps 4 and 5)

After all relevant quotations were coded for causal information, query reports were generated for each transcript. The reports contained quotations, associated codes, and the code notes comments containing causal structures in causal-loop diagram notation.

Based on the notes in the query report, freehand sketches were created for each coded feedback loop. These sketches were drawn using a tablet and stylus so they could be easily edited and digitally archived, although pen and paper would have also been sufficient. This analysis also allowed for identification and recording of modeler hypothesis structures_feedback loops or archetypes that were compatible with the source data, but were not directly generated from it. These hypothesis structures are akin to memoing in qualitative analysis (Strauss 1987; Birks 2008) and are a way for researchers to document their evolving understanding of the data. After the loops were identified, the causal links from the freehand sketches and query report were transferred to a causal mapping table. 


\subsubsection{Creation of causal mapping tables (step 6)}

A table was compiled containing information about variables and causal links. Separate tables were created for each interview using Excel. The table followed the format prescribed by Kumu for uploading data for visualization, which includes variable names, connection valence, and descriptive text and tags for both individual variables and links (see Appendix D for an example). Quotation numbers were included in descriptions of each variable and link. ATLAS.ti attaches quotation numbers to coded segments of text that appear in every form of data output, including the coding window and query reports, which aids in navigating source data.

Several tags were created in the causal mapping tables in Excel to enable easier navigation of data after maps were generated. Tags were created corresponding to the type of code used in generating that causal link (e.g., link, loop, etc.). Tags corresponding to a multi-level theoretical framework relevant to the subject matter of the interviews were also applied. Finally, tags were also included indicating whether a variable or connection were implied and whether the link involved a delay.

Each causal link and variable identified in the loop sketching phase was recorded in the causal mapping table according to the procedure outlined above. Causal links identified in the coding phase were then transposed from the query report generated by ATLAS.ti to the table in Excel. During this process, variable names 
were refined for clarity and consistency, often deferring to names identified during the loop sketching phase.

After all variables and connections were added, a final review was made to combine synonyms and check for typographical errors. The existence of separate query reports with coding notes and tables created a paper trail documenting the modeler's choices (e.g., combining variables).

3.5.4. Generation of causal-loop diagram from causal mapping table (steps 7 and 8)

The causal mapping table was then uploaded to Kumu for visualization of the causal-loop diagram using their causal-loop design template. An initial layout of the model was automatically generated by the software and pinned to enable custom changes to the position of variables and connections within the diagram. A single Kumu map was created for each interviewee's data.

The positioning of variables and connections within the diagrams was changed by the analyst to align with the norms outlined in Table 7. Loop variables were arranged in circles with curved arrows and exogenous variables were placed at the periphery, connected to loops with straight arrows when possible. Isolated variables were clustered and placed at the periphery. Variables covering similar themes were clustered into regions of the diagram. Delay symbols were added to connections tagged with "Delay." 
After positioning variables and connections, the diagram was reviewed for several types of necessary edits. If any remaining synonyms were identified, model sections were combined. In some models, certain causal links were rendered moot by other causal structures that conveyed the same idea in more detail. In some instances, new connections were made between model segments reflecting logical necessities. For any variables or connections added in the mapping phase, a tag of 'added' was included in the diagram in Kumu. Effort was made to minimize the amount of added variables and connections, in order to maintain fidelity to interviewees' mental models. Versions of the map prior to and following editing were preserved for future reference.

3.5.5. Use of causal-loop diagrams to understand mental models (step 9) The resulting causal-loop diagrams were analyzed individually and compared to each other to inform a future round of data collection in which models will be clarified based on structured follow-up interviews (see Chapter 4 for more detail). This step in the analysis is qualitative and guided by the relevant aims or research questions. Rather than using the models to quantify connections or generate estimated graphs of behavior, they were used as a different representation of qualitative data. The aim of this initial analysis was to identify information that needed to be elicited in the follow-up interviews. The prompts in Table 8 were used to guide this analysis. 
Table 8. Prompts for analyzing causal-loop diagrams generated from qualitative data

\begin{tabular}{|l|}
\hline Prompt \\
\hline Individual causal-loop diagrams \\
\hline Which feedback loops are included? Do any loops seem missing? \\
\hline Which model segments are isolated from other model segments? \\
\hline Which model segments are more complex than others? Less complex? \\
\hline How might sub-models be connected? \\
\hline Comparing causal-loop diagrams \\
\hline Which causal structures do the causal-loop diagrams have in common? Which are different? \\
\hline $\begin{array}{l}\text { Which causal-loop diagrams have more or fewer variables? Which have more complex causal } \\
\text { structures? }\end{array}$ \\
\hline $\begin{array}{l}\text { [lf diagrams for earlier data collection is available] How does this diagram compare with } \\
\text { diagrams from earlier points in time? }\end{array}$ \\
\hline
\end{tabular}

\subsection{Results}

The procedure outlined above, and summarized in Table 4, was used to identify causal structures in data from six practice facilitator interviews. Participants identified many similar variables when describing their mental models of how clinics successfully change, but the causal structures in which those variables were configured varied considerably. The resulting diagrams exhibited varying degrees of complexity. A selection of these diagrams can be found in Appendix E. Results included in this report showcase the application of the modeling process lined above; full results of this longitudinal study will be presented in a future article. 
Table 9. Characteristics of causal-loop diagrams gleaned from analysis of practice facilitator interviews.

\begin{tabular}{|c|c|c|c|}
\hline Participant & Variables & Causal links & Feedback loops \\
\hline 1 & 108 & 94 & 3 \\
\hline 2 & 79 & 44 & 0 \\
\hline 3 & 122 & 109 & 7 \\
\hline 4 & 92 & 84 & 5 \\
\hline 5 & 73 & 58 & 2 \\
\hline 6 & 77 & 59 & 2 \\
\hline
\end{tabular}

Participant mental models included many of the same variables, such as Clinician and staff (C/S) motivation and buy-in, PERC communication skills, and Health system affiliation. Due to differences in wording by participants, an attempt was made to harmonize variables names representing the same constructs during table compilation. If necessary, the paper trail for these judgements could be traced due to the use of quotation numbers and query notes.

The number of variables included in the diagrams ranged from 73 to 122, with three diagrams containing remarkably similar numbers of variables and links. It should be noted, however, that the number of variables present does not necessarily indicate a more complex mental model.

Causal links were by far the most frequent causal structure identified in the diagrams (see Table 9). While many of the variables were consistent across participants, the configuration of causal links connecting those variables varied 
considerably. Figure 13 illustrates how four participants conceptualized the variable Clinician and staff motivation and buy-in.

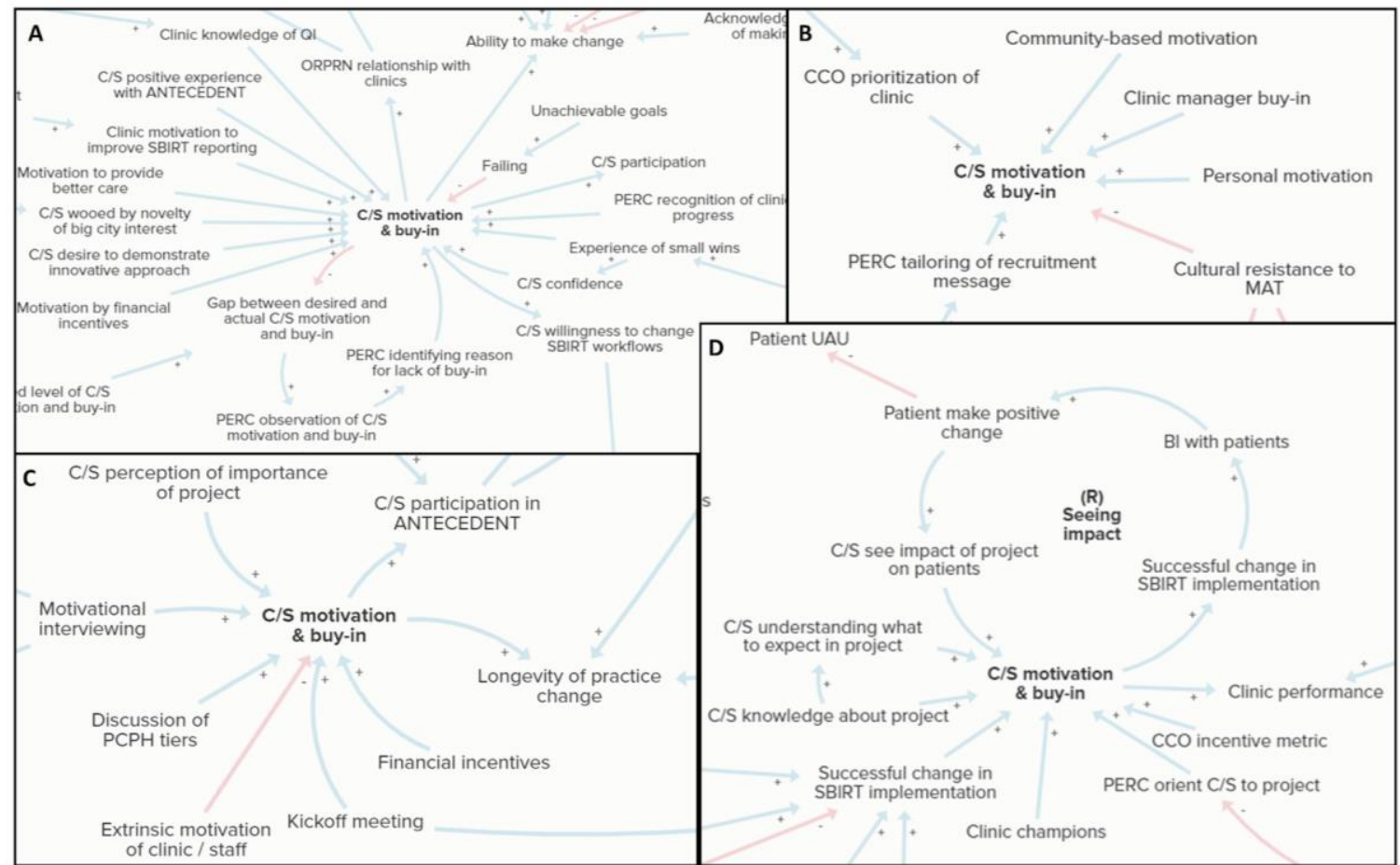

Figure 13. Causal structures surrounding clinician and staff motivation and buy-in ( $C / S$ motivation $\&$ buy-in) across four participants. The number of causal links and type of causal structures vary across diagrams. These diagrams were excerpted from larger diagrams summarizing participant mental models.

The diagrams also varied in the number of feedback loops identified. One diagram contained zero feedback loops, while the highest number was 7 . It is worth noting that the number of feedback loops does not necessarily reflect the complexity of the participant mental model; variation in speaking style, for example, could be a factor. Most feedback loops identified were reinforcing loops. To illustrate how data were coded and diagrammed, Table 10 shows each step in the process. In the quotation, one facilitator describes how seeing ways in 
which SBIRT activities can make positive impacts in patients' lives is important for maintaining long-term change.

Table 10. Data associated with steps in diagramming a feedback loop.

\begin{tabular}{|c|c|}
\hline Quotation: & $\begin{array}{l}\text { Interviewer: I'm wondering about change in the long-term. Not just signing up or } \\
\text { making some changes initially, but what helps clinics be successful in the long- } \\
\text { term and really make that sustainable? } \\
\text { Interviewee 5: Well, not to sound like a broken record, but I think that having that } \\
\text { buy-in is obviously really important and I think for the clinics to be able to see how } \\
\text { this impacts their patients positively is really important. So, seeing some results, } \\
\text { seeing the benefits of a patient that's been offered a brief intervention and takes } \\
\text { that to heart and does decide to make some changes or do whatever is a good } \\
\text { next step for them. I think that those are the aspects that might sustain that } \\
\text { change and encourage the clinics. So, I think seeing those results is going to be a } \\
\text { strong or a big motivator for the clinics in implementing the work and being } \\
\text { motivated to sustain that. }\end{array}$ \\
\hline Code: & Causal_feedback loops \\
\hline $\begin{array}{l}\text { Comment } \\
\text { : }\end{array}$ & $\begin{array}{l}\mathrm{C} / \mathrm{S} \text { buy-in building over time } \\
\mathrm{C} / \mathrm{S} \text { see impact of project on patients } \rightarrow \mathrm{C} / \mathrm{S} \text { buy-in } \rightarrow \text { Successful change in } \\
\text { long term SBIRT performance } \rightarrow \text { BI with patients } \rightarrow \text { patients make positive } \\
\text { change } \rightarrow \mathrm{C} / \mathrm{S} \text { see impact } \ldots \text { (reinforcing loop) }\end{array}$ \\
\hline Diagram: & 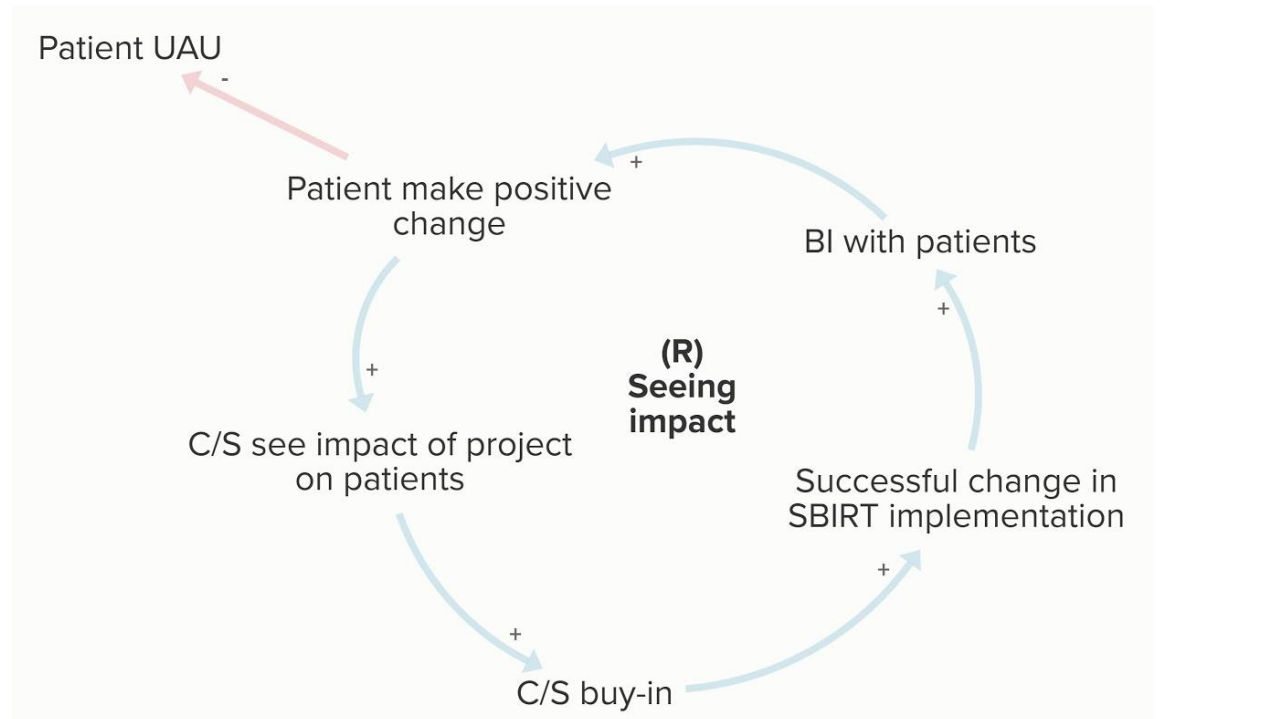 \\
\hline
\end{tabular}

Coding for implied information enabled the identification of causal structures that would have been ignored using link-based methods. A key topic of the ORPRN 
interviews was ways in which practice facilitators provide assistance to clinics so they can improve their SBIRT reporting and activities to meet benchmarks set by coordinated care organizations (CCOs), a type of Medicaid health plan in Oregon. The causal structure of this topic is a simple goal-directed balancing feedback loop: Current clinic SBIRT performance is compared to the CCO benchmark and activities such as changes in workflows or training are used to improve performance and reporting capabilities if needed (see Figure 14). In the setting of the interview, participants were able to correctly assume that the interviewer possessed this basic knowledge about SBIRT quality improvement based on how the interview was framed and the questions that were asked. While all of the participants referred to components of this causal structure and their responses were consistent with it, none of them explicitly identified each variable and causal link. Therefore, a causal structure that is arguably central to the participants' mental models would have been ignored using methods focused exclusively on causal links (e.g., Kim and Andersen 2012). 


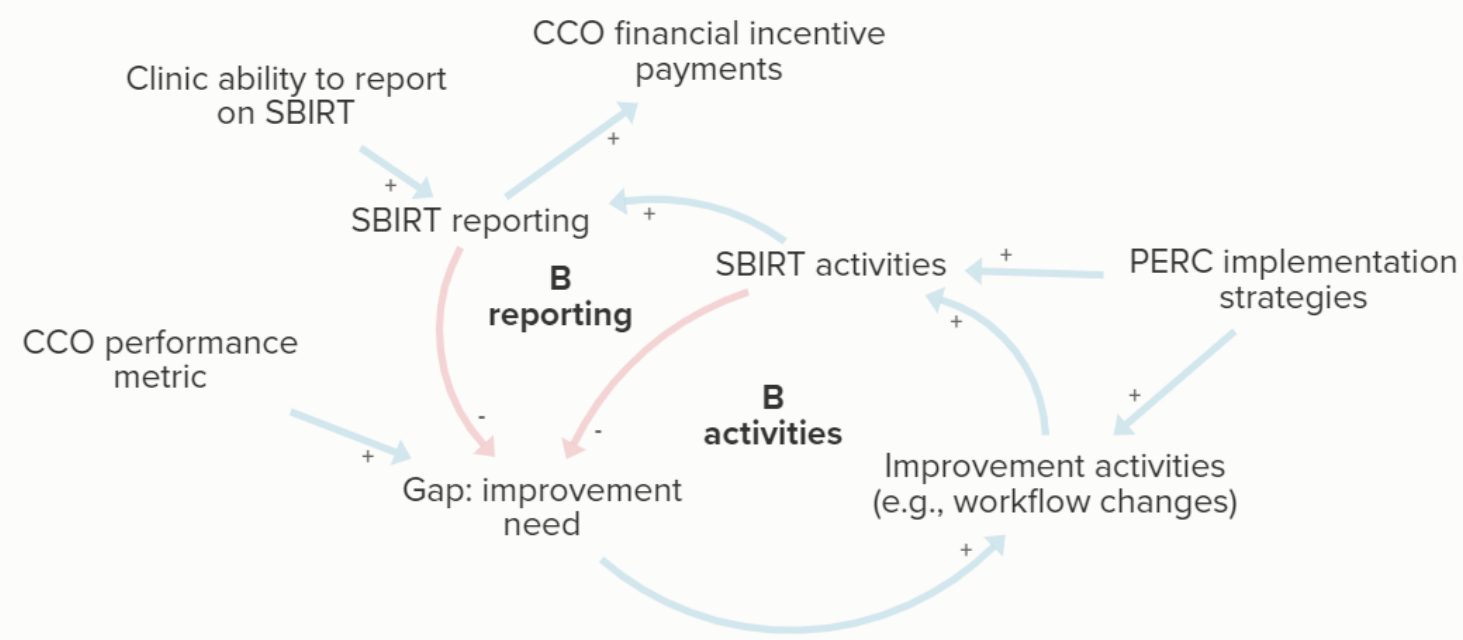

Figure 14. Goal-directed balancing feedback loops describing practice SBIRT quality improvement process. In the 'activities' balancing feedback loop, the gap between the adequacy of current clinic SBIRT activities (enabled by reporting) and the CCO performance metric constitutes an improvement need. PERCs (ORPRN practice facilitators) use implementation strategies to help the clinic with improvement activities (such as workflow changes) and to improve SBIRT activities (for example, through clinician training). SBIRT reporting is dependent on certain technical and staffing capacities and can result in CCO financial incentive payments. Distinct 'reporting' and 'activities' feedback loops illustrate that both are necessary to recognize and address improvement needs.

No archetypes were directly identified in the source data, but one was identified as a modeler hypothesis based on a combination of observations across participant diagrams. Many of the ORPRN interviews discussed clinic bandwidth as a factor limiting a clinic's ability to participate in quality improvement projects. Due to the similarity between that idea and the carrying capacity of a resource, I explored applying the carrying capacity archetype to the subject of the interviews. Figure 15 shows the generic carrying capacity archetype provided by Sterman (2000) compared with a causal-loop diagram created based on a modeler's synthesis of the source material. 


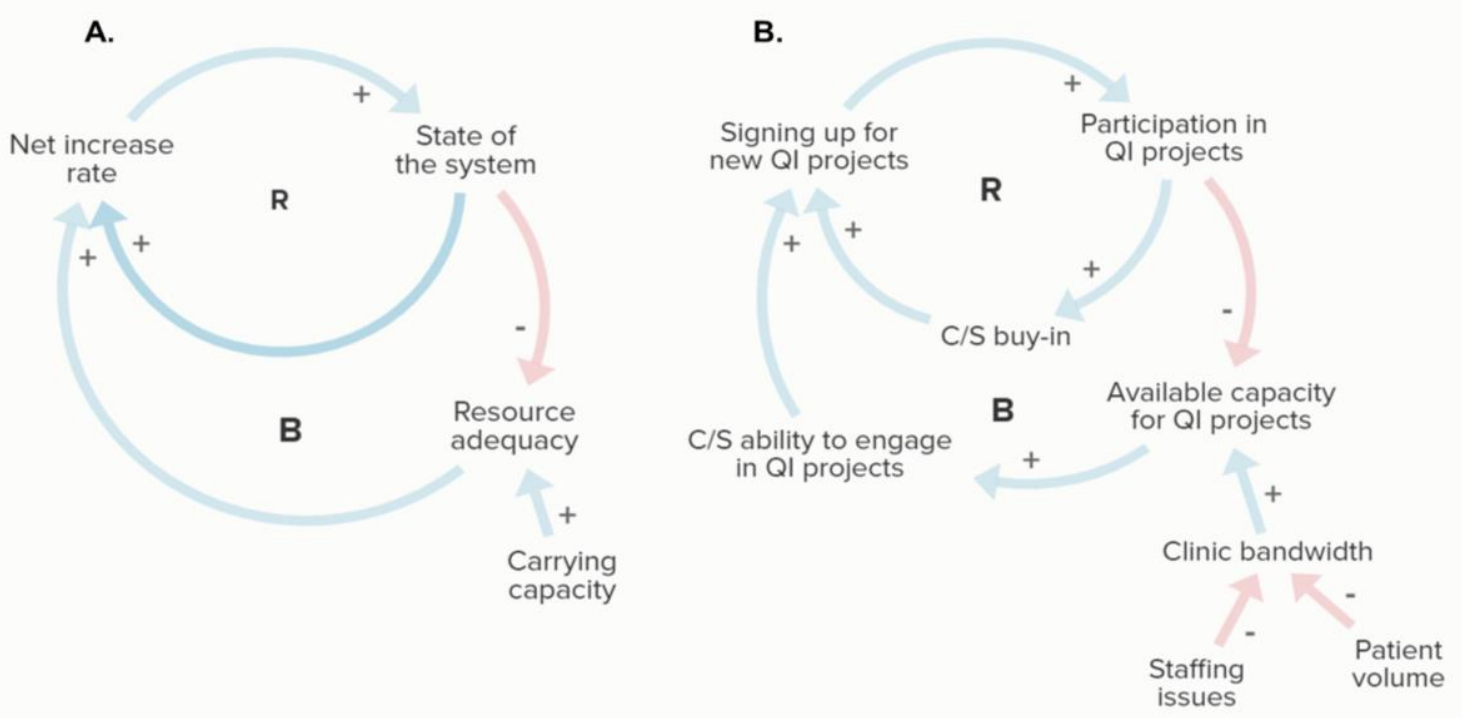

Figure 15. Modeler hypothesis diagram showing carrying capacity archetype applied to ORPRN case. Figure 15A describes the carrying capacity archetype adapted from Sterman 2000. A net increase rate improves the state of the system, which in turn further increases the net increase rate, forming a reinforcing feedback loop. An improved state of the system compromises resource adequacy, which decreases the net increase rate, forming a balancing feedback loop. Resource adequacy is limited by carrying capacity. The behavior for the archetype is an s-shaped curve, in which exponential growth turns to slow progression toward an upper limit (the carrying capacity). Figure 15B describes the same dynamics. Signing up for new QI projects results in more participation in QI projects and more clinician and staff buy-in, leading to more project signups-a reinforcing loop. More participation in QI projects leads to less capacity and ability to engage in them, which leads to less sign-ups. The carrying capacity variable in this scenario is clinic bandwidth, which is influenced by staffing issues and patient volume in this model.

\subsection{Discussion}

\subsubsection{Summary of findings}

In this research, an improved method for identifying causal structures in qualitative data was illustrated using a sample case. Diagrams describing practice facilitators' mental models of clinical practice change illustrated the process and product of this analysis. The method successfully produced diagrams representing participant mental models that could be analyzed and compared. The diagrams produced in this analysis largely consist of fragmented 
causal structures and variables, supporting the need for follow-up interviews to clarify and streamline the causal models (see Chapter 4).

Different numbers of variables, causal links, and feedback loops observed in the diagrams across participants could be understood to reflect differences in participant mental models (e.g., between novices and experts), speaking styles, or inconsistent application of the analysis method. Follow-up interviews or triangulation with other data collection methods (e.g., participant review of the diagram) may control for variations in speaking style (i.e., how explicitly a participant describes their mental model). The use of multiple analysts in the identification of loops during query review and during diagram editing may improve reliability.

\subsubsection{Advantages and limitations of approach}

The frequency, manner, and timing of modeler input in the process of diagram development represent a key difference between the approach outlined here and prior approaches. In methods presented by Kim and Andersen (2012) and subsequent researchers (Turner et al. 2013; Biroscak et al. 2014), the modeler assembled coherent causal-loop diagrams from causal links that had been identified and entered into a table. Larger causal structures, then, are created by the modeler without consulting directly with the source text. In the modified procedure outlined in this article, causal structures are identified during coding and query review, which encouraged greater focus on these elements and 
enabled much of the model design decisions to take place during a close reading of the source text. This centering of analysis around the data is in line with principles of qualitative analysis (Ritchie and Lewis 2003; Ezzy 2013; Strauss 1987; Braun and Clarke 2006) and builds credibility in modeling. While valuable to the resulting model, coding for multiple types of causal structures and model components is more complicated than coding for only causal links and requires training in causal-loop diagram modeling. However, fluency in system dynamics is also required for other methods for generating models from qualitative data.

The use of CAQDAS—in this case, ATLAS.ti-eased the process of tracking model components to source material and modeler contributions, enabled analysis at the quotation level, and allowed secondary causal mapping analysis to build on existing qualitative analysis. Integrating prior qualitative codes and causal mapping codes into the same file also enables querying across analysis types. For example, causal structures related to certain research questions or topics within the data could be easily extracted for analysis. The use of visualization software for mapping the causal-loop diagrams eased the process of model construction and enabled selective display of certain variables for analysis. Reliance on CAQDAS and visualization software, however, may present financial barriers to researchers and require some expertise in those platforms. 
In prior work, Kim and Andersen (2012) used identification numbers for specific claims in the source text as well as separate identification numbers for specific connections in the model, resulting in a large quantity of identification numbers to keep track of. Identification tags were also tracked manually-a laborious process. By using CAQDAS software to automate the generation of quotation numbers and data visualization software to automate the attachment of information to model components, record keeping is considerably less onerous. The use of quotation numbers also means that multiple components can get tied to the same quotation, creating a grouping of components associated with a certain part of the participant narrative. This grouping allows for the tracking of implicit components and enables selective display of grouped components using the data visualization software, allowing for greater contextualization during analysis. Coding by quotation allowed for navigating the text at a level of comprehension defined by the interviewee.

Freehand sketching feedback loops based on source text provided an opportunity for identifying key implicit variables and precisely naming variables based on their function within the loops. The use of freehand sketching to identify loops during analysis is in line with standard methods of creating qualitative system dynamics models (Hovmand 2014; Sterman 2000; Anderson and Johnson 1997) and provided an opportunity to name explicit and implicit variables in the feedback loops. By putting this loop sketching phase early in the model creation process, the modeler could base the causal structures on a close 
reading of the source text. Early loop sketching also allowed precise variable names to be created that could be used in later phases of causal mapping table generation.

Attention to implied variables allowed for the identification of many feedback loops that would have been missed using a method that only coded for causal links, such as those used by Kim and Andersen (2012), Turner and colleagues $(2013 ; 2014)$, and Biroscak and colleagues (2014). Nearly all of the feedback loops identified using the improved method contained implied variables. This illustrates a potentially important advantage to this approach.

The analysis would have been considerably less time intensive if the CAQDAS had been capable of logging causal links—perhaps as a type of linked codeand generating a causal mapping table for export into the visualization software. Automation of this process, however, would eliminate the additional reflection and analysis that comes with making and reviewing coding notes.

The identification of modeler hypothesis structures can help the researcher understand their own mental model and guide subsequent rounds of data collection. Sketching of modeler hypothesis structures provides a way to document modelers' understanding of the target system. For example, the carrying capacity model that was identified in this research (see Figure 15) was used to inform a follow-up round of interviews (see Chapter 4). 
The data analyzed for this study was produced in semi-structured interviews that focused in part on practice facilitators' mental models of clinical practice change and therefore contained information relevant for mapping mental models. Greater clarification and probing designed to elicit information about causal structures, however, might have produced even richer data for causal-loop diagramming.

The longitudinal study is still ongoing, so the overall contribution of this analysis to this research has not yet been determined and will be addressed in subsequent articles.

\subsubsection{System dynamics applications}

Because it provides a way to systematically generate causal-loop diagrams from qualitative data while tracking the modeler's contribution, the method outlined here has the potential of adding rigor to the use of interviews for system dynamics model building. This method could be used to augment group model building processes. Models gleaned from preliminary individual interviews could form the basis of a participatory modeling session with stakeholders (Vennix 1996), or interviews could be used when synchronous participation is impractical or impossible (Luna-Reyes and Andersen 2003). This method could also be used as part of an alternative strategy to group model building. As mentioned earlier, semi-structured interviews are a broadly accessible mode of data collection, both for the interviewee and interviewer, while group model building can present 
logistical and accessibility barriers to participation. Moreover, a process of analyzing, comparing, and synthesizing individual mental models may be preferable to a group modeling process, depending on the goals of the modeling project.

\subsubsection{Qualitative applications}

This approach to generating causal-loop diagrams from qualitative data has possible uses in qualitative and mixed methods research. The production of causal-loop diagrams from qualitative data can be seen as a kind of translation or conversion of information from one form to another and could therefore be used as an alternative way to identify themes or insight from qualitative data. Navigating qualitative data in this way could be useful for identifying patterns in stakeholder mental models in the context of community engagement, program evaluation, or collaborative partnerships. It could also augment standard qualitative research in the social and behavioral sciences in arenas such as health services. As noted in the preceding chapter, improved methods for generating causal-loop diagrams from qualitative data could be useful for incorporating stakeholder, staff, and expert perspectives in theory-based evaluation.

3.7.5. Ramifications for automated model generation

Automatic methods of extracting causal information from text are being developed using natural language processing, but they are currently far from reliable (Jung 2017; Doan et al. 2019). The idea of using these automated 
methods for generating causal-loop diagrams from text data has been floated (Owen et al. 2018) and would indeed be transformative if successful. Possible applications include analysis of qualitative data for research and synthesis of scientific literature for review. This type of machine learning-based analysis, however, would likely rely on identification of individual causal links rather than causal structures. As illustrated in this research, exclusive reliance on causal links obscures implicit causal structures in natural language. The prospect of automatically generating causal-loop diagrams from text data, therefore, may be further in the future than previously thought.

\subsubsection{Future research}

Future research could identify effective strategies for collecting data suitable for this type of analysis by drawing from best practices in qualitative interviewing and system dynamics modeling. Guidelines for creating interview guides designed to elicit causal structures would be particularly useful (see Chapter 4). Future studies could also develop methods for visually communicating the degree of support within data behind individual causal claims in causal loop diagrams, so that causal links mentioned repeatedly are visually distinct from links that are mentioned fewer times. Additionally, follow-up research could adapt this method for research questions seeking to summarize the mental models of groups of individuals, such as stakeholders. Finally, future studies could systematically compare the method outlined here with other approaches to generating complex 
systems diagrams from qualitative data, as can be found in recent literature on fuzzy cognitive mapping (Alibage 2020; Alizadeh and Jetter 2017).

\subsection{Conclusion}

Prior methods for generating causal-loop diagrams from qualitative data made strides to increasing transparency and credibility in system dynamics modeling, but did not account for implied variables and structures, which prevented feedback loops and archetypes from being identified during analysis. By leveraging software to improve tracking and streamline visualization, the improved method outlined here enables transparent and systematic identification of larger causal structures (feedback loops and archetypes) in qualitative data. These improvements further enhance transparency and credibility, but the approach is still relatively time intensive and requires fluency with identifying causal structures. This method could be applied to standard system dynamics projects, qualitative research, and evaluations in which the benefits outweigh the effort required. Software designed to meet the needs of this analysis could streamline the process considerably. Future research should enhance strategies for data collection designed to elicit data suitable for model building and examine the value of this approach to the proposed applications. 
4. Paper \#3: Advancing interview-based methods for mapping mental models using causal-loop diagramming

Target journal: Systems Research and Behavioral Science

\subsection{Abstract}

Participatory methods are the gold standard for reliably reproducing the mental models of stakeholders or experts in system dynamics modeling. The system dynamics field has a robust knowledge base about group model building—a type of participatory modeling — but this approach is not always feasible or appropriate. Individual interviews have long been used in system dynamics, but methods for gleaning model components from qualitative data have only recently been explored. Purposive text analysis and its subsequent adaptations are promising and would be strengthened by an iterative framework for data collection tailored to the needs of modeling. This research draws from system dynamics, qualitative methods, and realist evaluation to propose interview-based data collection strategies for mapping mental models using causal-loop diagramming. This method is designed to increase transparency and rigor in the use of interviews for system dynamics and has a variety of potential applications.

\subsection{Introduction}

Doyle and Ford (1999) defined a mental model as a "relatively enduring and accessible, but limited, internal conceptual representation of an external system whose structure is analogous to the perceived structure of that system." Understanding mental models is a key part of qualitative research and system 
dynamics modeling, with many applications. Causal-loop diagramming-a method from the field of system dynamics—has been used to represent mental models due to its relatively simple format capable of describing complex dynamics (Sterman 2000). Iterative, participatory methods are preferred for capturing mental models because they allow the opportunity for stakeholders or experts to be involved in the modeling process (Jones et al. 2011).

The system dynamics literature has an extensive body of knowledge about group model building - a collection of hands-on methods for involving groups of participants in model creation (Vennix 1999; Hovmand et al. 2012; Richardson and Andersen 1995; Rouwette et al. 2002). The product of a group modeling process is a causal-loop diagram or simulation model that represents the shared mental model of the group that created it. Participating in group model building provides an opportunity for participants to refine their own mental models, actively steer model design, and build consensus and rapport with fellow participants—seen as a key benefit to group model building (Hovmand et al. 2012; Vennix 1999).

Group model building, however, is not always feasible or appropriate (Meinherz et al. 2016). The method typically requires a series of synchronous, in-person meetings with a consistent group of participants and a skilled set of facilitators (Tàbara et al. 2008; Olabisi 2013; Schmitt Olabisi et al. 2010). While skilled modeler-facilitators will seek to make the experience accessible for participants, 
the activities and notation used in group model building are typically unfamiliar to participants may "not be contextually appropriate due to lower levels of education, literacy, numeracy, and analytical capacity" (Valcourt 2020). People's abilities to comprehend a system dynamics model and compare it to their own existing mental model, which is part of group model building, also likely varies. When presented with a model, particularly one supported by other participants, it can be easy to agree without meaningfully engaging with the material $-a$ phenomenon known as confirmation bias (Nickerson 1998). The process and design choices made by facilitators also shape the end product. Moreover, because the method is designed to enable groups to interactively co-produce a shared mental model, it is not necessarily well suited to reliably eliciting individual mental models.

Methods for mapping mental models that leverage the strengths of established interview methods are needed. A key strength of traditional semi-structured qualitative data collection methods, such as interviews and focus groups, is that they let participants speak freely, in their own words, about a phenomenon of interest (Weiss 1995). There is considerable precedent in the system dynamics literature for using interview data in the process of model building (Luna-Reyes and Andersen 2003), but processes for systematically generating models from qualitative data have not been widely established. Kim and Andersen (2012) presented a procedure based on grounded theory for coding causal links in qualitative data and assembling system dynamics models from those links, which 
has been termed purposive text analysis. This method has since been streamlined and adapted by other researchers (Turner et al. 2014; Biroscak et al. 2014). While this approach has made significant strides in increasing the transparency of generating a model from qualitative data, existing methods are insufficient because they don't allow for individual data collection, don't capture implicit info, and focus on links instead of causal structures (see Chapter 3). Moreover, this literature does not provide guidance about effective data collection strategies for mental model elicitation.

In linguistics, it is widely acknowledged that human language consists of both explicit and implicit communication (Yus 1999). The maxims of cooperative conversation proposed by Grice (1975) indicate that people try to be just as informative as required, but not more. The norms for directness in communication is also highly culturally dependent (Gudykunst et al. 1988; Nelson et al. 2002). We know from systems education that people do not typically think of systems in terms of fully formed models (Doyle 1997). In qualitative research, part of the skill and art in effective interviewing and analysis involves listening for information that is expressed implicitly (Cruz 2008). Therefore, interview-based mental model elicitation should be carefully planned. People cannot simply be asked to share their mental model; they must be actively guided to reveal (or construct) it. 
This research addresses the need for interview-based strategies for eliciting mental model data suitable for causal-loop diagramming. A participatory approach is outlined, with a focus on planning and conducting the interviews. Results of several studies using this protocol will be presented in future publications.

\subsection{Interviewing approaches}

This research draws from interviewing practices from several areas: qualitative research, system dynamics, and realist interviewing. Strategies used in these fields overlap, but have distinct angles and philosophical underpinnings.

\subsubsection{Qualitative interviewing}

A wide variety of approaches are used for interviewing in qualitative research; a comprehensive review is beyond the scope of this paper. Individual qualitative interviews typically consist of one-on-one synchronous conversations between an interviewer and a participant (also called an interviewee) (Crabtree and Miller 1999; Braun and Clarke 2006; Gubrium and Holstein 2001; Kvale and Brinkmann 2009). Semi-structured interviews are a common format. Using this approach, the interviewer prepares an interview guide outlining key questions and probes (follow-up questions) used to steer the conversation (Creswell and Báez 2020).

During the interview, the interviewer uses the guide to make sure that all important topics are covered, but questions are not necessarily asked verbatim and in order. The questions in semi-structured interviews are open-ended and designed to let the participant talk freely about certain topics of interest. The 
wording of questions is important and can limit or distort participants' replies (Crabtree and Miller 1999). Probes associated with questions in the interview guide can pertain to additional relevant information or clarification (Creswell and Baez 2021).

Constructivist assumptions are well suited to qualitative research because they situate the focus of the interview on the lived experience of the participant. By asking open-ended, neutral questions as a deliberate naiveté or "amiable incompetent," the interviewer seeks to minimize the influence of their own biases or opinions and instead center the experience of the participant (Kvale and Brinkmann 2009; Sapsford and Abbott 1992). By encouraging the participant to speak freely and openly about the subject matter in their own words, the interviewer can reasonably assume the qualitative data they collect is a reliable reflection of the participant's mental models.

\subsubsection{Interviewing in system dynamics}

Although well suited for producing data for standard qualitative analysis, constructivist interviewing approaches do not provide the foundation necessary for model building, which typically involves an iterative process in which the modeler plays an active co-creator role. An ontology that accounts for the modeler's role in model creation is needed. 
Originating amid postwar efforts to predict and control an increasingly complex sociotechnical world, system dynamics emerged from engineering and the cybernetics efforts of the 1960s with positivist assumptions: a real world exists, and we can observe it reliably enough to create models of it (Pruyt 2006).

Although positivism and postpositivism still shape the methods and assumptions of system dynamics, most mainstream system dynamicists today could be called critical realist or critical pluralist (Pruyt 2006). This approach is described by Pruyt (2006) as a blend of realism and constructivism:

The ontological position of such critical pluralist system dynamics is realist (an external real world exists), whereas its epistemological position is subjective (the real world can only be accessed via subjective mental models). So, it is assumed that there is an external reality that could only be known to a certain extent, because it is necessarily approached by means of subjective mental models.

The methodological ramifications of this paradigm are not widely discussed in the system dynamics literature, but are evident in the field's choices of methods: System dynamicists construct models that approximate real-world systems in order to better understand them. System dynamics models are now widely seen as reflections of the mental models of their designers rather than direct reflections of target systems, although positivist assumptions still linger in the field (Pruyt 2006).

Although the literature on designing interview protocols for system dynamics modeling is not robust (Luna-Reyes and Andersen 2003), there is some 
guidance to be found. Martinez-Moyano and Richardson (2013) used a threephase process in which preliminary individual interviews guided an asynchronous web-based "meeting" (similar to an interactive survey) in which data were collected from participants. Those data were collected and used to inform a facilitated in-person discussion among participants. Luna-Reyes and Andersen (2003) orient system dynamicists to qualitative research and provide the following advice for utilizing interviews for model development:

During and after the interview the researcher looks for dynamic hypotheses-stories about how dynamic systems work-and tests these hypotheses by asking for more specific information, or presenting the developing causal story and asking the respondent to comment upon it.

\subsubsection{Realist interviewing}

The realist school of philosophy integrates positivist ontological assumptions with a constructivist understanding of the role of individual experience in shaping our understanding and experience of that reality (Mukumbang et al. 2019). Realist evaluation, a theory-based approach first defined by Pawson and Tilley (1997), emphasizes the importance of context in assessing program outcomes. Rather than evaluating whether a program "works," realist evaluation examines "what works, for whom, in what respects, to what extent, in what contexts, and how?" (Flynn et al. 2019).

The purpose of interviewing in realist evaluation is to "inspire/validate/falsify/modify" hypotheses about how programs or interventions are supposed to work (Pawson 1996). Typically, the interviewer presents this 
hypothesis to the participant for their feedback-an approach that contrasts with more common constructivist interview approaches that assume a naive stance for the interviewer (Manzano 2016). Through iterations of dialogue between interviewer and participant in realist interviewing, the researcher's hypothesis is corrected or refined (Mukumbang et al. 2019). This process, termed the teacherlearner function, is a key component of realist interviewing that involves the interviewer and interviewee taking turns 'teaching' their mental model to each in a process of progressive refinement (Manzano et al. 2016).

Based on a review of interview approaches within realist evaluation, Manzano (2016) proposes a three-phase process for realist interviewing: theory gleaning, theory refining, and theory consolidation. Later refined by Mukumbang and colleagues (2019), this process begins with an exploratory interview to "identify and link intervention modalities, actors, relevant context, generative mechanisms and intended/observed outcomes." In the second interview, the researcher presents their theory and elicits feedback from the participant. The third interview provides an opportunity to fine-tune theories with select participants. Byng and colleagues (2005) utilize diagrams to visually summarize study results. This basic three-interview structure has been adapted to the protocol presented in this study. 
4.4. Overview of interview-based process for diagramming mental models The method outlined in this paper borrows from the interviewing approaches outlined above to specify an iterative interview-based method for articulating individuals' mental models in a research or evaluation setting. This process is designed to bring the researcher's understanding of the participant's mental model into alignment with the participant's actual mental model through a series of iterative steps.

Some of the example interview questions used in this article come from an implementation science study to assess how practice facilitators tailored implementation support to clinics in a project to improve screening and treatment of unhealthy alcohol use in primary care. This case was featured in Chapter 3. Other example questions were written for this article to illustrate aspects of the proposed process.

In the initial phase of the approach, a semi-structured interview provides an opportunity for the participant to describe their mental model in their own words. The researcher adopts the deliberate naiveté stance of the constructivist interviewer. Through careful causal coding and analysis of the interview transcript, the researcher constructs a hypothesis model representing the participant's mental model as a causal-loop diagram. This diagram is used to guide a follow-up interview designed to address gaps and uncertainties about the hypothesis model. The causal-loop diagram is then refined based on input from 
the second interviews. In an optional third phase, participants are invited to compare the hypothesis models and apply them to different scenarios. This iterative process borrows its three-part structure from realist interviewing (Manzano 2016; Mukumbang et al. 2019). The tailored follow-up interviews also use a realist orientation, in which the interviewer solicits structured feedback about a proposed hypothesis or theory.

Although the process outlined below could be conducted with a single participant, the description is written under the assumption that the researcher would want to elicit the mental models of multiple participants as part of a research or evaluation endeavor.

Table 11. Proposed interview-based protocol for mapping individual mental models

\begin{tabular}{|c|c|c|c|}
\hline Step & Approach & Input & Output \\
\hline $\begin{array}{l}\text { 1. Define system of } \\
\text { interest and } \\
\text { preliminary indicators, } \\
\text { boundaries, and } \\
\text { participants }\end{array}$ & $\begin{array}{l}\text { Determine which } \\
\text { boundaries will be set } \\
\text { by researchers and } \\
\text { which by participants }\end{array}$ & $\begin{array}{l}\text { Goals of evaluation, } \\
\text { client input, evaluator } \\
\text { expertise }\end{array}$ & $\begin{array}{l}\text { Evaluation design } \\
\text { adhering to needs of } \\
\text { evaluation and best } \\
\text { practice for methods }\end{array}$ \\
\hline $\begin{array}{l}\text { 2. Conduct initial } \\
\text { interviews }\end{array}$ & $\begin{array}{l}\text { Semi-structured, } \\
\text { constructivist } \\
\text { interviews following } \\
\text { open-ended guide }\end{array}$ & $\begin{array}{l}\text { Evaluation plan, } \\
\text { interview guide, } \\
\text { participants (e.g., } \\
\text { stakeholders, experts) }\end{array}$ & Interview transcripts \\
\hline $\begin{array}{l}\text { 3. Analyze initial } \\
\text { interviews, draft } \\
\text { diagram \& identify } \\
\text { gaps }\end{array}$ & $\begin{array}{l}\text { Causal structure } \\
\text { mapping as outlined in } \\
\text { Chapter } 3\end{array}$ & $\begin{array}{l}\text { Interview transcripts, } \\
\text { analyst expertise }\end{array}$ & $\begin{array}{l}\text { Initial causal-loop } \\
\text { diagrams, tables, } \\
\text { researcher } \\
\text { notes/models }\end{array}$ \\
\hline $\begin{array}{l}\text { 4. Conduct follow-up } \\
\text { interviews }\end{array}$ & $\begin{array}{l}\text { Guided, realist } \\
\text { interviews following } \\
\text { tailored guide }\end{array}$ & $\begin{array}{l}\text { Tailored interview } \\
\text { guide, analysis of initial } \\
\text { diagrams, sample of } \\
\text { initial participants }\end{array}$ & Interview transcripts \\
\hline $\begin{array}{l}\text { 5. Analyze follow-up } \\
\text { interviews and refine } \\
\text { model }\end{array}$ & $\begin{array}{l}\text { Causal structure } \\
\text { mapping as outlined in } \\
\text { Chapter } 3\end{array}$ & $\begin{array}{l}\text { Interview transcripts, } \\
\text { analyst expertise, initial } \\
\text { diagrams from step } 3\end{array}$ & $\begin{array}{l}\text { Refined diagrams, } \\
\text { tables, researcher } \\
\text { notes/models }\end{array}$ \\
\hline
\end{tabular}




\begin{tabular}{|l|l|l|l|}
\hline $\begin{array}{l}\text { 6. Conduct } \\
\text { participatory review } \\
\text { (optional) }\end{array}$ & $\begin{array}{l}\text { Based on group model } \\
\text { building }\end{array}$ & $\begin{array}{l}\text { Participant group, } \\
\text { modeler-facilitators, } \\
\text { refined diagram } \\
\text { draft(s), facilitation plan }\end{array}$ & $\begin{array}{l}\text { Session transcripts, } \\
\text { feedback about model } \\
\text { revision }\end{array}$ \\
\hline $\begin{array}{l}\text { 7. Refine model and } \\
\text { study outputs }\end{array}$ & $\begin{array}{l}\text { Final revision and } \\
\text { reporting }\end{array}$ & $\begin{array}{l}\text { Feedback and } \\
\text { transcripts from } \\
\text { participatory review, } \\
\text { evaluation plan }\end{array}$ & $\begin{array}{l}\text { Finalized diagram(s), } \\
\text { evaluation report, } \\
\text { supplemental } \\
\text { documentation }\end{array}$ \\
\hline
\end{tabular}

Figure 16 illustrates the steps outlined in Table 11 to highlight how investigator and participant mental model are brought into increasing alignment through the iterative, participatory process.

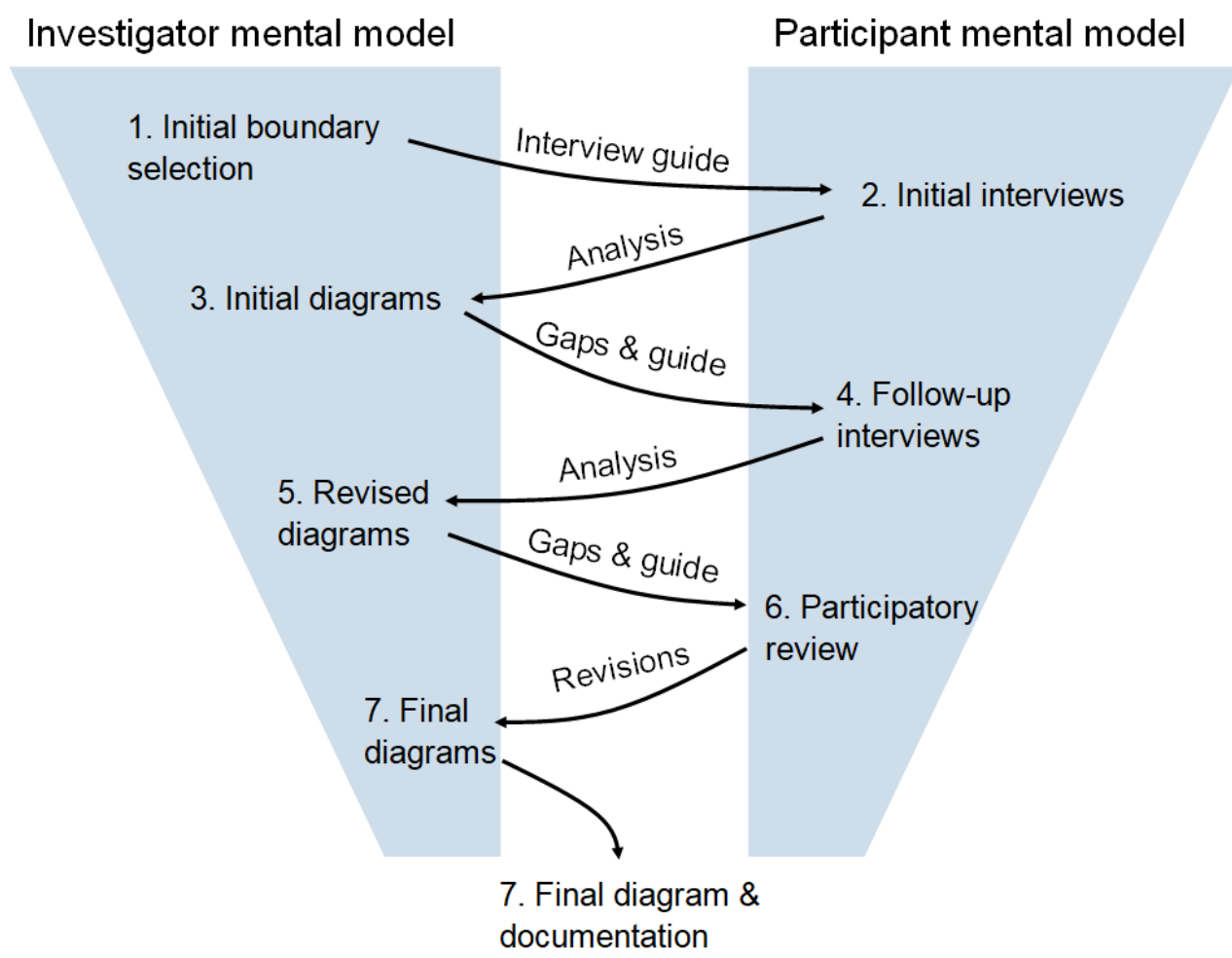

Figure 16. Iterative, participatory process for diagramming mental models. Numbered steps correspond to numbers in Table 11. Tools and strategies used to link steps are included alongside the arrows. 


\subsubsection{Boundary definition and planning}

To begin the process, the researcher first defines the research question(s), purpose of the interviews, target system, and participants, in collaboration with clients or other key stakeholders (step 1 in Table 11). They may also identify key variables and a system of interest. A system of interest, also referred to in the system dynamics literature as a target system, is a set of variables that interact to shape system behavior of interest to the researcher (Sterman 2000). System behavior is represented by key indicator variables showing change over time (Sterman 2000). Systems of interest are also defined by boundaries-what is endogenous, exogenous, or excluded to the system. The system of interest is typically identified by the researcher prior to data collection, although relevant indicator variables and boundaries might be refined by participants as the research progresses. The selection of participants is carefully determined by the researcher based on the aims of the study and should be inclusive of relevant perspectives. The guiding question when designing an interview-based model building study is "Whose mental model of what is being modeled? These decisions set the outer boundaries of the modeling project and are key to overall success.

In some projects, the key variables and behavior over time are clear from the research questions or modeling goals. A project seeking stakeholder perspectives about low colorectal cancer screening rates within a certain population, for example, has a clear problem definition and approximate behavior 
over time. In other situations, stakeholders might disagree about how to characterize a problem, or whether a problem even exists. These situations may be characterized as "wicked problems" (Rittel and Webber 1973; Head and Alford 2015). Other mental modeling projects may be exploratory and leave more room for participant definition of the problem.

Because the primary goal of these interviews is to elicit information suitable for causal-loop diagramming, the interview guides and strategies should be guided by an understanding of the components of these diagrams. Table 12 presents these components, including three types of causal structures (causal links, feedback loops, and archetypes). 
Table 12. Components of causal-loop diagrams

\begin{tabular}{|c|c|c|}
\hline Component & Description & Guiding Questions \\
\hline Problem & $\begin{array}{l}\text { Short description of the problem as } \\
\text { behavior over time for one or several } \\
\text { variables }\end{array}$ & $\begin{array}{l}\text { Defined either prior to data collection } \\
\text { by researchers or by participants }\end{array}$ \\
\hline & Factors relevant to the problem & $\begin{array}{l}\text { Which factors influence the } \\
\text { outcomes? What is relevant to } \\
\text { understanding the situation? }\end{array}$ \\
\hline Boundaries & $\begin{array}{l}\text { Distinctions between what is } \\
\text { considered inside the system and } \\
\text { outside it. Endogenous / exogenous / } \\
\text { excluded. }\end{array}$ & $\begin{array}{l}\text { Which factors serve a primary role? } \\
\text { What is outside the scope? }\end{array}$ \\
\hline & $\begin{array}{l}\text { Relationships between variables. } \\
\text { Directed with a valence, if possible. } \\
\text { Note delay if relevant. }\end{array}$ & $\begin{array}{l}\text { How do variables relate to one } \\
\text { another? }\end{array}$ \\
\hline & $\begin{array}{l}\text { When chains of causal links connect } \\
\text { back to a variable earlier in the chain. } \\
\text { Reinforcing or balancing. }\end{array}$ & $\begin{array}{l}\text { What are the goals of the system? } \\
\text { How do relationships between } \\
\text { variables produce system behavior? }\end{array}$ \\
\hline Archetypes & $\begin{array}{l}\text { Common configurations of causal } \\
\text { structures reflecting known patterns of } \\
\text { behavior (see Kim 1994). }\end{array}$ & $\begin{array}{l}\text { Does the participant narrative } \\
\text { resemble known archetypes? }\end{array}$ \\
\hline
\end{tabular}

A dimension of causal-loop diagramming that cuts across the components featured in Table 12 is the idea of multiple perspectives. In these interviews, the primary goal is to understand the participant's mental model, but it is helpful to recognize that not all actors will have the same perspective. Asking participants to comment on whether other actors would agree with their assessment can lead to a greater understanding of the participant's mental model of how those actors fit into the system. 


\subsubsection{Initial interviews}

In the first interview phase, the researcher conducts semi-structured interviews with participants to elicit their mental models about the problem of interest. These interviews capture the participants' views in their own words and are conducted using a constructivist qualitative approach. The data gleaned from these interviews serve as the foundation for the subsequent phases.

\section{Interview approach}

While the form of the initial interview is semi-structured with open questions, the interview guide is carefully designed so as to elicit the participant's rich description of their mental model about the phenomenon of interest. The interviews are typically conducted according to standard qualitative practice (by 1-2 people, in person or remote, recorded on at least two devices, and transcribed) (Crabtree and Miller 1999). Table 13 summarizes proposed design criteria for initial interviews, including description or purpose of the criteria and corresponding sources.

Table 13. Design criteria for initial interview

\begin{tabular}{|l|l|l|}
\hline Design criteria & Source & Description / purpose \\
\hline How to approach the interview & Qualitative research & $\begin{array}{l}\text { Allows participant to steer content of } \\
\text { the interview }\end{array}$ \\
\cline { 1 - 2 } Interviewer as naive learner & Qualitative research \\
participant's mental model & Qualitative research & \\
\cline { 1 - 2 } Constructivist orientation & System dynamics & $\begin{array}{l}\text { Focuses the interview on what is } \\
\text { relevant to understanding how } \\
\text { system behavior is produced; part of }\end{array}$ \\
\cline { 1 - 2 } $\begin{array}{l}\text { Problem-centric or behavior- } \\
\text { centric }\end{array}$ & \multicolumn{2}{|l}{}
\end{tabular}




\begin{tabular}{|c|c|c|}
\hline & & boundary setting \\
\hline \multicolumn{3}{|l|}{ What to ask } \\
\hline Introductory questions & Qualitative research & $\begin{array}{l}\text { Establish rapport, help participant } \\
\text { get comfortable and start talking }\end{array}$ \\
\hline Problem definition questions & System dynamics & \multirow[t]{3}{*}{$\begin{array}{l}\text { Get an initial understanding of } \\
\text { participant mental model }\end{array}$} \\
\hline Behavior over time & System dynamics & \\
\hline Key variables and relationships & System dynamics & \\
\hline Intervention leverage points & System dynamics & $\begin{array}{l}\text { If the mental model includes } \\
\text { interventions, which 'levers' are } \\
\text { those interventions trying to } \\
\text { influence? }\end{array}$ \\
\hline Outcomes and their precursors & Evaluation & $\begin{array}{l}\text { What are the system outcomes } \\
\text { during the status quo and for } \\
\text { proposed interventions? What leads } \\
\text { to those outcomes? }\end{array}$ \\
\hline \multicolumn{3}{|l|}{ What to listen for } \\
\hline $\begin{array}{l}\text { Coherent narrative or dynamic } \\
\text { hypothesis }\end{array}$ & Qualitative research & $\begin{array}{l}\text { Descriptions of how processes work, } \\
\text { cause-and-effect dynamics }\end{array}$ \\
\hline $\begin{array}{l}\text { Explicit references to causal } \\
\text { structures }\end{array}$ & System dynamics & $\begin{array}{l}\text { Explicit phrases such as "vicious } \\
\text { cycle" or identifying goals }\end{array}$ \\
\hline Implied causal structures & System dynamics & $\begin{array}{l}\text { Variables or structures can be } \\
\text { implied through discussions of } \\
\text { behavior or chains of events }\end{array}$ \\
\hline Boundaries & System dynamics & $\begin{array}{l}\text { Factors that play key roles vs. } \\
\text { factors that are less important or } \\
\text { outside the scope }\end{array}$ \\
\hline \multicolumn{3}{|l|}{ How to probe } \\
\hline Clarifying what is heard & Qualitative research & $\begin{array}{l}\text { Interviewer verifying whether they } \\
\text { understand correctly }\end{array}$ \\
\hline Ask why and how & Qualitative research & $\begin{array}{l}\text { Clarify precursors, mediating } \\
\text { variables, and consequences }\end{array}$ \\
\hline Explore what-ifs & Qualitative research & $\begin{array}{l}\text { Ask if same dynamics apply in other } \\
\text { contexts, or if other events had } \\
\text { occurred }\end{array}$ \\
\hline
\end{tabular}

\section{How to approach the interview}

In the initial round of data collection, the researcher approaches the task as a learner. A constructivist approach allows the researcher to center the experience 
of the participant and minimize their own influence. The goal of the initial interview is to generate a rich narrative describing the mental model of the participant.

While the philosophical stance of the initial interview is constructivist in the sense that the participant's perspective and experience is centered, interviewing for the purpose of mapping a mental model involves more pointed follow-up probe questions than a standard qualitative interview.

The participant's mental model of the problem or system behavior of interest should guide the interview. If understanding an intervention is a goal of the project, the interview addresses how that intervention acts upon the pre-existing system.

What to ask

Planned questions in the initial interview guide largely resemble questions in standard qualitative research and are focused on eliciting a rich participant narrative of the problem or situation as defined in the project.

Initial interviews consist of questions designed to establish a welcoming environment and get the participant to describe their mental model about the phenomenon of interest in some detail. Open-ended introductory questions build rapport, get the participant talking, and can orient the interviewer to the scope of the participant's knowledge. Some examples of introductory questions include the following: 
- Can you start by telling me a bit about the work you do with clinics?

- Tell me a bit about your background and how you came to work at [organization name].

- What has your experience as a practice facilitator been like so far?

- Tell me about what a typical day is like for you.

Questions designed to elicit descriptions of the problem definition serve to clarify the system of interest and establish behavior over time for key system variables. Depending on the boundaries defined prior to the interview, questions can be open-ended or prompt the participant to respond within a certain problem definition. A question such as, "What is the situation like for CRC screening for the rural Medicaid population in Oregon? Is it going well or not so well?" provides a boundary in regards to target population and prompts the interviewee to define the problem or situation. A question such as, "In your opinion, why have CRC screening rates remained low for the rural Oregon Medicaid population?" sets a boundary for the target population and problem behavior, and asks the interviewee to identify factors driving that behavior. To elicit descriptions of key variables and relationships, the interviewer can build on the participant's problem definition. The purpose of these questions is to take stock of the dynamics responsible for the problem behavior.

If a particular intervention is being examined, as in an evaluation, questions can be aimed at identifying the leverage points corresponding to the intervention, as illustrated in these questions pertaining to an at-home fecal immunochemical test (FIT) for colorectal cancer:

- Can you tell me about the rationale behind the Mailed FIT program? How is it designed to improve screening rates? 
- You mentioned that patient reluctance regarding colonoscopy is the largest barrier to improving colorectal cancer screening rates. How does the Mailed FIT intervention address that barrier?

Questions could also examine desired future outcomes and work back toward potential precursors, as is done in group model building. Some sample questions include:

- If the program is successful, what might screening rates be in five years? - What would need to happen for that goal to be achieved?

What to listen for

While the questions outlined above could be found in a typical semi-structured qualitative interview, strategies for listening and probing outlined in this protocol more customized to model structure. In general, the interviewer listens for a coherent narrative describing how combinations of variables shape system behavior. If explicit or implied references to causal structures are made, they should be noticed by the interviewer. Because larger causal structures like feedback loops and archetypes are often implied (see Chapter 3 ), they require close attention and follow-up questions to be clarified. To listen for causal structures, the interviewer should have an understanding of how they operate (see Table 12).

Because variables and causal links are ubiquitous in participant narratives, the interviewer may not need to make a particular effort to elicit them. Asking the participant to compare the importance of different variables or relationships may be helpful. Identifying feedback loops is a central part of this interview strategy 
and is less straightforward than listening for variables and causal links. Loops can be identified using two primary cues: usage of certain terms and descriptions of behavior.

Reinforcing feedback loops, in which the effects of variables are amplified through circular causation, demonstrate increasing or decreasing exponential behavior (see Figure 17 for generic structures and examples). References to "vicious" or "virtuous" cycles imply reinforcing feedback. Descriptions of exponential growth may include terms such as growing, cascade, runaway, getting out of hand, building on itself, amplify, or out of control.

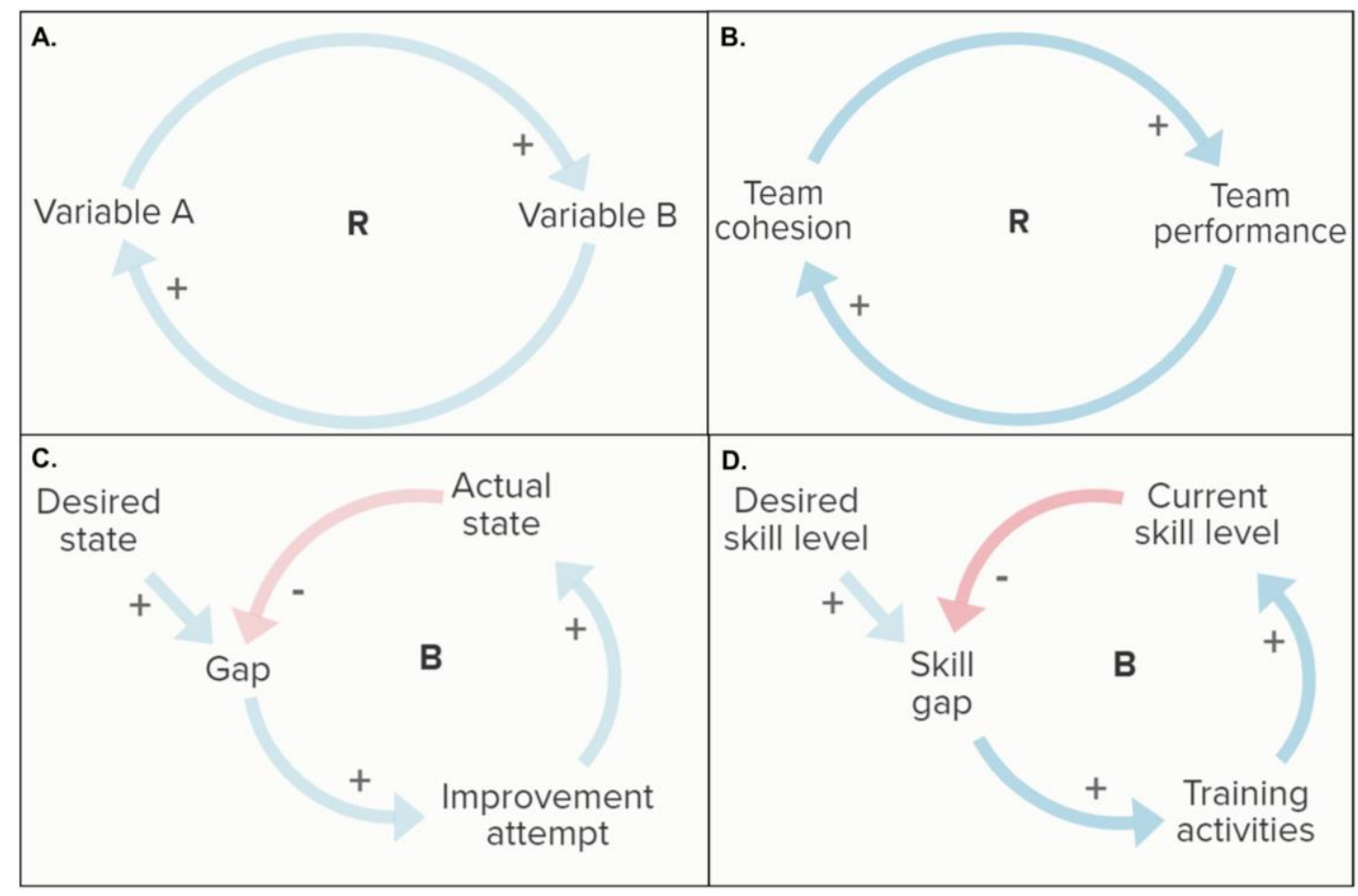

Figure 17. Generic structures and examples of reinforcing and balancing feedback loops

Balancing feedback loops describe behavior that trends toward an implicit or explicit set point or goal over time (Sterman 2000; see Figure 17). References to 
working toward a goal imply this structure and often contain a fair amount of implied information in natural language. When someone says they are trying to lose weight or saving up for vacation, they are communicating a goal-directed balancing feedback structure. We understand that they have a goal, a current state, and a gap between the two that inspires some kind of ameliorative action, even if those variables and relationships are not explicitly mentioned. Other references to balancing behavior include terms like stay in balance, reach homeostasis, stabilize, recover, heal, even out, keep in check, rein in, keep in line, or reduce tension.

Archetypes are certain causal structure configurations that have been identified in systems science as common across many contexts (Kim 1994; Kim and Anderson 2007; Senge 2010). The tragedy of the commons, which describes overexploitation of a common resource, is a widely known systems archetype. The dynamics described by other archetypes are likely more commonly observed in the world, but are not widely known by name. The escalation archetype describes an arms race situation in which cutthroat competition works against the interests of both parties. In the success to the successful archetype, unequal initial conditions create a path dependence in which resources flow from the least to most powerful. The shifting the burden archetype describes how short-term fixes can cause unintended consequences and interfere with more meaningful long-term solutions. Figure 18 illustrates the causal structures of three common systems archetypes. Identifying archetypes "in the wild" requires familiarity with these structures; archetypes may be evident only on reflection during analysis. 


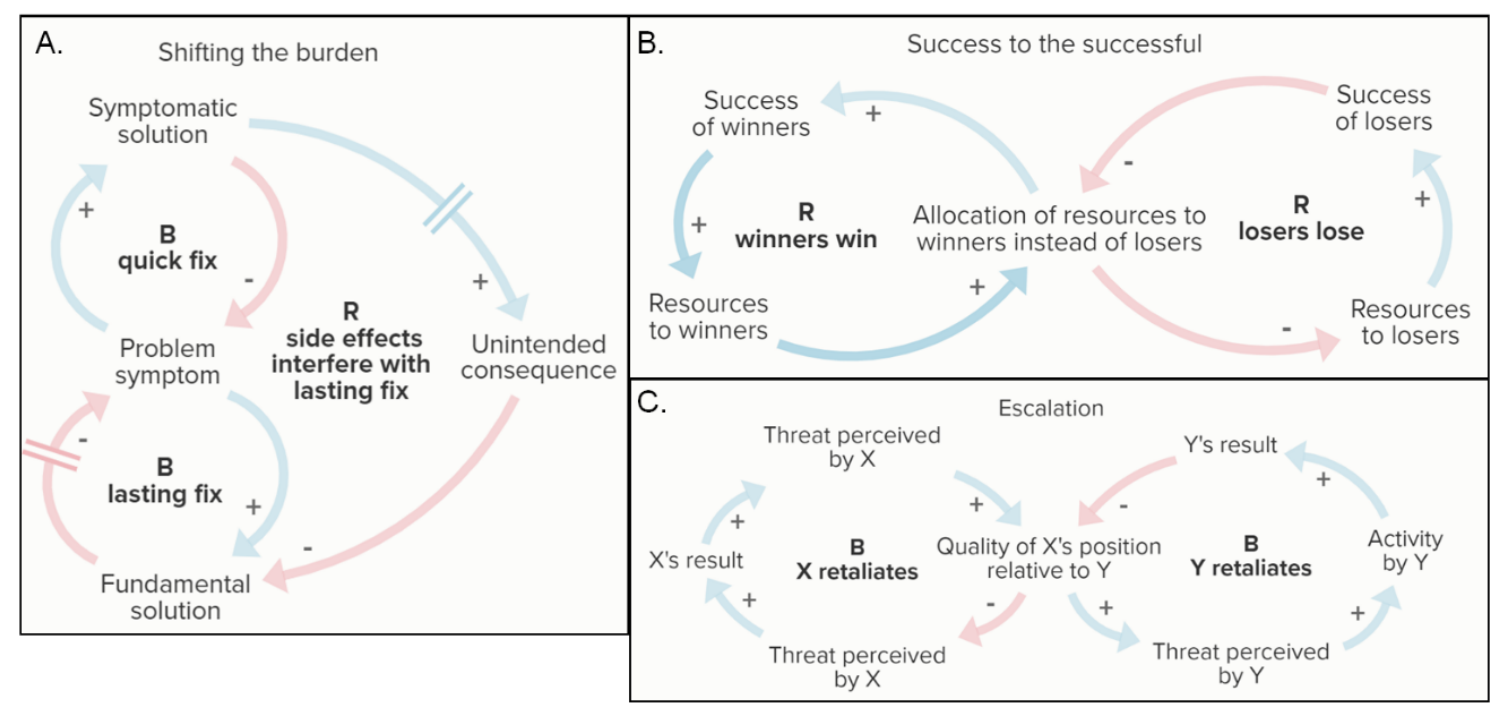

Figure 18. Three common systems archetypes: shifting the burden (Figure 18A), success to the successful (Figure 18B), and escalation (Figure 18C). Adapted from Kim 1994. $R=$ reinforcing loop; $\mathrm{B}=$ balancing loop.

\section{How to probe}

Follow-up questions (also known as probes) are important for model-building interviews because they allow an opportunity for clarification and for the interviewer to steer the participant toward providing the needed information. In a model-building interview, the interviewer should listen attentively and be prepared to guide the discussion in a more active way than is common for qualitative interviews.

The most simple probes to elicit underlying dynamics are Why? and How? These questions can prompt an interviewee to explicitly state information that they had previously implied, and can help get at other variables driving the behavior. In addition to clarifying what was heard, probes can be used to identify precursors, 
mediating variables, and consequences or effects. Asking participants to connect variables in this way provides valuable causal information. Probing questions can also explore whether identified variables or relationships apply in other contexts, or whether they would have happened differently in other scenarios (counterfactuals).

Because participants are likely unfamiliar with the components of causal-loop diagrams, probing questions should not ask directly about "causal links", "feedback loops", or "archetypes." Questions should be worded in a way that is accessible to the participant. For example, to ask about other causal links associated with a certain variable, the interviewer might ask, "Do any other factors come into play?" If a reinforcing feedback loop is suspected, a probe could be, "So it sounds like $X$ and $Y$ amplify or reinforce each other?" or, "Over time, does that become a vicious cycle?" If a participant refers to a goal, the interviewer could ask about parts of a goal-directed balancing feedback loop: "Can you tell me more about the steps you're taking to work toward that goal?" or, "How will you know if you're making progress?" These questions could help identify the improvement attempt, current state, and gap variables included in Figure 17.

Because the structure of archetypes differ, there is more than one way to ask about them. Paraphrasing the participant's narrative into a structure aligned with the suspected archetype could be one strategy. For example, to ask about the shifting the burden archetype, an interviewer could ask, "So you're saying that 
the Veterans Administration allowed Veterans to receive care at community clinics to increase access to care, but over time, that policy hurt the VA's ability to provide adequate care, which worsened overall access?" A follow-up question could inquire about more fundamental solutions to increasing Veteran access to care: "How might the VA design a system of healthcare delivery that improves overall access to care in the long term?" These questions are less open-ended than questions used in standard qualitative research and are designed to yield model-specific information.

\subsubsection{Mapping analysis}

The data produced by the initial interviews should be well suited for being coded and mapped using the causal structure mapping method outlined in Chapter 3. In this method, interview transcripts are coded using software to identify causal structures using detailed notes, feedback loops and archetypes are identified using close reading of the source text and notes, a table is created compiling all identified causal information. The table is uploaded and rendered using visualization software and the resulting diagram is formatted and revised for cohesion and to reduce repetition. The final diagram typically consists of fragmented causal segments. The diagram is analyzed to identify gaps in knowledge to inform future data collection. In an abbreviated version of this process, a more informal review of the recording or transcript could be used to inform modeling. 


\subsubsection{Follow-up interviews}

In the recommended protocol (see Table 11), step 4 follow-up interviews borrow from realist interviewing to focus on aspects of the model that are missing or need to be clarified. These interviews are guided by the output of the mapping analysis outlined in the previous step. Participants may include the entire group interviewed in the initial step, or a smaller number, based on identified knowledge gaps. If clusters of similar mental models have emerged, the interviewer may choose to follow up with a selection of participants representing each cluster. The main goal of these interviews is to try to turn the map segments identified in the first round of interviews into coherent models, or to identify contradictions preventing the articulation of a coherent model.

Follow-up interviews are valuable because they allow an opportunity for clarification and to increase ownership. Interview guides for follow-up interviews closely follow the output and needs from previous analysis. Questions in the guide are more targeted than the open-ended questions in the initial interviews and could address gaps, inconsistencies, verification of observed structures, or connection of causal structures. Some example questions used in follow-up interviews in the case described in Chapter 3 are listed below:

- At our last interview, you talked a lot about the value of communication skills and maintaining good communication with clinics. Can you talk a bit about how that makes a difference when you're working with a clinic?

- When we last talked, you said that building intrinsic motivation is important for longevity. Can you say more about that? How do you tell if a clinic is intrinsically motivated? 
- In our initial interview, you talked about factors that can impact clinic bandwidth. Can you describe how bandwidth affects implementation?

The above questions inquire about precursors and consequences of variables identified in the initial interview, as well as connections between subsections of the diagram. Questions inquiring about feedback loops or archetypes could follow the approach outlined for probes in section 4.4.1.

Transcripts of the follow-up interviews are coded and notes generated through close analysis of the source text using the process outlined in section 4.4.2. Rather than generating and uploading a table, causal information is incorporated from the coding notes into the initial diagram in the visualization software by the analyst. Quotation numbers are associated with causal links to preserve tracking. If multiple conflicting maps have been produced, these are described or merged, depending on need. Remaining gaps or contradictions are identified and used to inform the next phase of the research. If a participatory model review is not being used, the results are summarized to accompany the model.

\subsubsection{Participatory model review}

In the last (and optional) step in the interview-based modeling process, the draft model is presented for feedback and final editing to participants. This could be done as a group or individually, depending on need. The researcher walks the participants through the diagram, being sure to "tell the story" behind the diagram to aid comprehension. Methods from group model building can guide this process. Depending on feasibility and the aims of the research, this step could be 
skipped or done asynchronously using a video walkthrough and accompanying documentation. The design of this session is flexible based on the needs of the individual or group. Diagrams produced during this analysis could also be used as inputs to more robust group model building processes.

\subsection{Potential applications}

The iterative, participatory approach to mapping mental models with causal-loop diagramming outlined in this protocol could be used or adapted in a variety of applications, such as system dynamics modeling, program evaluation, and qualitative research. Illustrations for each of these areas follow.

\subsubsection{System dynamics modeling}

This protocol for gleaning causal-loop diagrams from stakeholders or experts in an individual interview format could strengthen the credibility and accuracy of system dynamics models and broaden the range of possibilities for participatory modeling. Using interview strategies designed to elicit information suitable for system dynamics modeling may bring the resulting models into closer alignment with participants' mental models. Carefully tracking input from participants and modelers during analysis may increase credibility and accuracy. Individual interviews could be used as a precursor to group model building or alongside it when synchronous in-person meetings are not feasible or desired. 


\subsubsection{Program evaluation}

In a review of how causal-loop diagramming has been applied to theory-based evaluation, Chapter 2 identified that evaluators basing their diagrams on secondary analysis of prior evaluation data felt constrained by the scope of the source material. Engaging stakeholders and experts proactively during needs assessment using the interview strategies outlined in this paper could support program designs that are richer and better suited to underlying problems and provide a framework for evaluation along the life of the program.

\subsubsection{Qualitative research}

In recent years, interest has grown in finding ways of analyzing qualitative data that are more systematic (Schnieder and Wagemann 2012) or participatory [Van der Merwe 2019; Catalani and Minkler). Causal-loop diagrams systematically generated from qualitative data present a potentially innovative way of analyzing and communicating participants' mental models.

\subsection{Discussion}

The protocol presented in this research addresses a need for structured guidance for designing interview-based model building processes to produce diagrams of mental models. An iterative, seven-step participatory process adapted from the three-phase realist interviewing method engages participants in a series of interviews customized to the needs of causal-loop diagramming. Individual engagement with participants in a flexible and familiar in-person or 
remote interview format allows for data collection that is versatile and accessible for participants. The systematic nature of the proposed method meets a need for rigor and transparency when using interviews as inputs for modeling. The method, however, is time consuming and requires interviewer familiarity with the components of causal-loop diagramming.

\subsubsection{Experimental comparison with group model building}

Prior research has attempted to compare the effectiveness of interview-based methods for mapping mental models and standard group modeling methods, but the ways in which these comparisons are made can be questioned. In a recent study, Valcourt and colleagues. (2020) found that group modeling generated causal-loop diagrams with more feedback loops than causal-loop diagrams generated from standard semi-structured individual interviews analyzed using Kim and Andersen's (2102) purposive text analysis. On this basis, the authors conclude that "GMB produces higher quality models than can be obtained by eliciting individual mental models in isolation" (Valcourt et al. 2020). The authors acknowledge, however, that participants were prompted to identify causal relationships between variables in the group modeling session, but not in the individual interviews. It is therefore unsurprising that the causal-loop diagrams generated in the group setting contained more causal links and feedback loops. The structured, iterative interview-based process outlined in this research could be used to guide fairer comparisons between interview-based and group modeling approaches. 


\subsubsection{Choosing between interview-based modeling and other methods}

Interview-based model building as outlined here may be integrated into standard modeler-led system dynamics modeling or group model building, as outlined in section 4.5 , or it can be used as a standalone method. Table 14 compares interview-based modeling with these approaches and standard semi-structured qualitative interviews to illustrate key differences between these methods.

Table 14. Comparison of features of modeler-led system dynamics, group model building, interview-based model building, and standard semi-structured qualitative interviews, with positive characteristics highlighted in green

\begin{tabular}{|l|l|l|l|l|}
\hline Feature & $\begin{array}{l}\text { Modeler-led } \\
\text { system } \\
\text { dynamics } \\
\text { modeling }\end{array}$ & $\begin{array}{l}\text { Group model } \\
\text { building }\end{array}$ & $\begin{array}{l}\text { Interview-based } \\
\text { model building } \\
\text { (outlined in this } \\
\text { paper) }\end{array}$ & $\begin{array}{l}\text { Standard semi- } \\
\text { structured } \\
\text { qualitative } \\
\text { interviews }\end{array}$ \\
\hline $\begin{array}{l}\text { Accessible for different } \\
\text { participant abilities }\end{array}$ & N/A & No & Yes & Yes \\
\hline $\begin{array}{l}\text { Elicits rich descriptions of } \\
\text { individuals' mental models }\end{array}$ & No & No & Yes & Yes \\
\hline $\begin{array}{l}\text { Produces a causal-loop } \\
\text { diagram }\end{array}$ & Yes & Yes & Yes & No \\
\hline $\begin{array}{l}\text { Builds group rapport and a } \\
\text { shared mental model }\end{array}$ & No & Yes & No & No \\
\hline Requires modeling skill & Yes & Yes & Yes & No \\
\hline $\begin{array}{l}\text { Carefully tracks modeler } \\
\text { influence }\end{array}$ & No & No & Yes & No \\
\hline
\end{tabular}

Interview-based model building is suitable for research questions that seek to understand participants' mental models about a system of interest. Because mental models are about how a system works, the participants' understanding of how the factors driving outcomes relate to each other must be relevant. Standard qualitative research can describe participants' attitudes or feelings about a topic, prior experience, or values and beliefs. Modeler-led system dynamics modeling can produce a model of a system using available data to explore hypotheses 
about how certain interventions may affect system behavior. This approach is best used when the structure of the model is noncontroversial and data to operationalize it are widely available. Group model building is most appropriate when team cohesion and shared understanding is necessary for group decision making.

\subsubsection{Future research}

Future research should test the effectiveness and feasibility of this proposed method in various settings, such as research and evaluation. Specifically, the interview strategies outlined in this paper may enable people using causal-loop diagrams for theory-based evaluation (as in Chapter 2) to develop more effective models. Interview-based modeling could also be compared with standard qualitative research. Comparisons should also be made with group model building to identify situations in which each approach is best suited. After the method is refined and its relative strengths and limitations are known, guidelines for use in research and evaluation can be updated and expanded.

\subsection{Conclusion}

The use of interviews to inform system dynamics modeling is common, but systematic, rigorous, and transparent methods for designing appropriate interviewing strategies have been lacking. This article describes an iterative and participatory seven-step process to elicit and diagram mental models using causal-loop diagramming. Interview-based model building could improve data collection and broaden the base of participation for existing modeling approaches 
and open a door to new ways of analyzing qualitative data in research and evaluation settings. Future research should examine the effectiveness of this protocol in a wide variety of settings. 


\section{Synthesis}

\subsection{Contributions to knowledge}

The research documented in this dissertation offer three primary contributions: 1) a review of how causal-loop diagramming has been used to depict complexityaware program theory in the context of program evaluation; 2) an improved process for generating causal-loop diagrams from qualitative data; and 3) an iterative, interview-based framework for mapping mental models designed to elicit data suitable for causal-loop diagramming. These results contribute to several broader themes: the importance of implied information in mapping mental models, the modeler as co-creator, causal-loop diagrams as a problem-centric approach, and causal-loop diagrams as representations of knowledge.

\subsubsection{Capturing implied information in mapping mental models}

The paper presented in Chapter 3 adapts existing methods for generating causal-loop diagrams from qualitative data to account for implied causal structures. Chapter 4 presents strategies for designing and conducting interviews to elicit this information. Together these methods are intended to strengthen the ability of researchers to access participants' mental models by enabling the identification of larger causal structures such as feedback loops and archetypes. This method for identifying causal structures through close reading of qualitative data is novel and stands in contrast to other methods in which causal structures are assembled post hoc from explicitly stated causal links (e.g., Kim and Andersen 2012; Renmans et al. 2017). This approach reinforces the role of 
qualitative analysis in identifying causal structure from text data and presents an added challenge for proposed automated analysis methods.

\subsubsection{Modeler as co-creator}

The methods outlined in Chapters 3 and 4 were designed to account for and track the role of the modeler in diagram development. In qualitative research, the perspectives and abilities of the researcher are acknowledged as assets to the analysis process (Braun and Clarke 2016). To varying extents, researcher biases are minimized in qualitative research using methods such as multiple coders. Attention to the role of the modeler in shaping model content is comparatively understudied in systems science. This research is intended to strengthen the credibility of the proposed approach through more precise tracking of modeler contributions and encouraging of close reading of source text at key decision points (such as identification of feedback loops).

\subsubsection{Causal-loop diagrams as a problem-centric approach}

The review in Chapter 2 illustrates a contrast between methods to diagramming that center the intervention (e.g., standard pipeline logic models) and the use of causal-loop diagrams to center an understanding of the problem or aspects of the status quo responsible for producing undesirable system behavior. This contrast may be indicative of a broader distinction between intervention-centric and problem-centric or systems-based approaches that can be seen in a variety of settings, such as evidence-based medicine. Figure 19 illustrates these two approaches. 
A

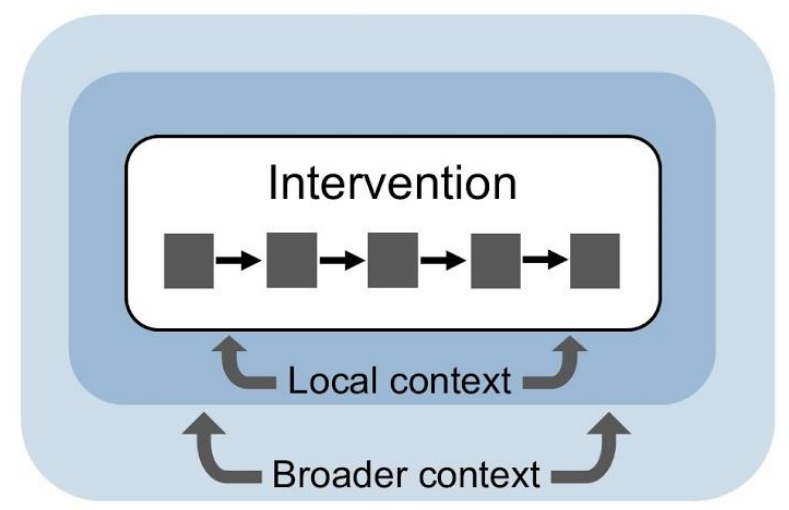

B

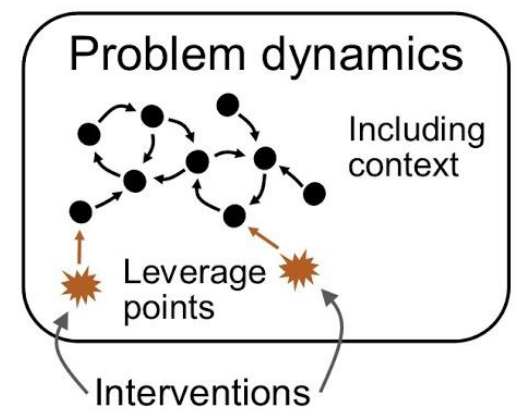

Figure 19. Intervention-centric and problem-centric approaches to understanding systems change.

Although Figure 19A and Figure 19B both include interactions between intervention and context, Figure 19A nestles the intervention into inner and outer context. This conceptualization is in line with many logic model approaches (as described in Chapter 2) as well as the consolidated framework for implementation research (CFIR) from implementation science (Damschroder et al. 2009). While situating the intervention into layers of context is intuitive, it forces aspects of context into an exogenous role, implying that context influences or acts on the intervention.

Figure 19B, in contrast, describes a problem-centric approach characteristic of systems science (Sterman 2000). In this approach, a model is constructed that represents key dynamics of a system in the world that produces a certain (typically problematic) behavior. A key distinction is that these problem dynamics existed prior to or without the intervention. Model construction is guided by the 
question How do variables interact to produce the problematic behavior? After a credible representation of the system (as defined by the problem) is in place, leverage points can be identified. Leverage points are places in the system at which certain changes have the potential of leading to systems change (Meadows 2008). The field of systems science has categorized different types of leverage points based on the kind of structural change they produce (Meadows). In a systems approach, interventions are designed based on an understanding of a problem's causal structure and potentially effective leverage points. In this way, interventions can be understood as acting on existing systems (Renmans et al. 2020).

The distinction between the two orientations is potentially consequential to how interventions or programs are conceptualized, planned, adapted in practice, and evaluated. Because causal-loop diagrams and other systems models constitute a dynamic hypothesis of how variables interact to produce certain system behavior (Sterman 2000), they require a meaningful understanding not only of which aspects of context are important, but how they matter. When designed well, systems models center structural aspects influencing and constraining how human actors behave as well as leverage points associated with those structures-an approach that can align with stakeholder perspectives. 


\subsubsection{Causal-loop diagrams as representations of knowledge}

To date, causal-loop diagrams have most commonly been used for the purpose of system dynamics model building or for teaching basic system dynamics concepts (Sterman 2000; Anderson and Johnson 1997; Richardson 1986; Lane 2008; Wheat 2007; Aubrecht et al. 2019). In both applications, diagrams are judged by their ability to describe how interactions between a small number of variables generate the behavior seen in a target system. As such, they should be simple and describe only key dynamics relevant for describing the behavior of interest (Sterman 2000), reflecting the norms of simulation modeling.

Causal-loop diagramming, however, has uses beyond the service of simulation modeling. This dissertation research explores how it can be used for describing individuals' mental models (Chapter 4) and program theory in organizations (Chapter 2)-two applications with needs and norms distinct from that of simulation modeling or education. While simple models with clear feedback dynamics are important for standard uses, comprehensiveness may also be valuable in these newer applications. When diagramming causal structures identified in qualitative data for the purpose of mapping mental models, fragmented segments and variables represent opportunities for clarification, but are not necessarily faults of the diagram. Mental models are messy, and are not necessarily complete and coherent (Meadows 2008). When comparing diagrams of mental models in the context of qualitative research, differing degrees of fragmentation may itself be a finding. In the context of theory-based evaluation, 
the inclusion of certain content, such as program activities or outcomes, may be essential. As causal-loop diagrams are applied and adapted for new purposes, new guidelines and norms for their development and use should be identified.

The shift from assessing a model based on how reliably it can reproduce system behavior (as is common in computational system dynamics modeling) to seeing it as a representation or translation of ideas is a significant paradigm shift. Causalloop diagrams can serve as a snapshot of a person's mental model, a distillation of the causal claims embedded in their narrative. As with any translation, fidelity to the source text is key. Careful tracking from source to diagram, and of modeler influence, makes the process transparent and reduces bias.

\subsection{Implications / Significance}

By advancing methods for using causal-loop diagrams for complexity-aware program theory and gleaning diagrams from qualitative data, this research improves the transparency and accuracy—and therefore credibility—of the practice of mapping systems qualitatively. Enhancing its rigor has the potential not only to strengthen validity for individual studies in which it is used, but also to contribute toward expanding the scope of potential applications for causal-loop diagramming.

One potentially innovative application is the use of causal-loop diagramming for synthesizing different forms of knowledge and evidence into 'living' decision tools 
for contexts such as program management and implementation science. In implementation science, for example, there has been a recent call for strategies for adapting evidence-based clinical interventions to local contexts (Morrison et al. 2009; Cohen et al. 2008). Causal-loop diagrams could provide a common 'language' in which to integrate clinician and patient perspectives, practitioner mental models, and published scientific evidence into a dynamic hypothesis. The recommendations for using causal-loop diagrams for complexity-aware program theory in Chapter 2 could inform how implementation scientists develop and use a qualitative systems model to inform decision making, and the interview-based approach for generating causal-loop diagrams from qualitative data outlined in Chapters 3 and 4 could facilitate incorporating practitioner and stakeholder perspectives. Due to the ability of causal-loop diagrams to clarify the dynamics of problematic behavior embedded in the status quo prior to intervention, the proposed approach might be well suited for identifying mechanisms underlying health disparities and appropriate corresponding adaptations.

\subsection{Limitations}

The research included in this dissertation has several limitations. I had originally planned to test the protocol and analysis method outlined in Chapters 3 and 4 at two local nonprofit organizations in spring 2020 , but was unable to do so due to research restrictions associated with the COVID-19 pandemic. Pilot testing the strategies from Chapter 4 and additional testing of the method from Chapter 3 
would allow for refinement and provide valuable data about feasibility and utility across settings.

A limitation of the approaches outlined in each paper is that they require expertise in modeling with causal-loop diagrams. This skill set is not yet common, and involves training and a degree of creativity, according to Sterman (2000). The development of guidelines and detailed methods serves to make the approach more transparent and accessible, but some fluency is necessary in order to recognize feedback loops and design and analyze diagrams. If these approaches gain popularity for research or evaluation, training will need to be developed and made available.

This research draws on evidence and practices from diverse areas of literature and during its development I had to make many decisions about where to draw the boundary of what to review or include. Many types of causal mapping, for example fuzzy cognitive mapping (Özesmi and Özesmi 2004; Jetter and Kok 2014), would surely provide useful input for refining the methods presented here. Thematic analysis, a type of qualitative analysis, has been used to create fuzzy cognitive maps, for example (Alibage et al. 2018). Other approaches in qualitative research, evaluation, and other fields such as psychology may be similarly instructive. A proper review of these methods, however, was outside the scope of this research. 


\subsection{Future research}

The papers included in this dissertation provide a variety of opportunities for future research. The review of causal-loop diagrams for effective program theory identified in Chapter 2 could be used to develop and test strategies for effective use of this approach in multiple program contexts. Further testing of the method outlined in Chapter 3 would allow an opportunity for refinement and validation. Strategies such as multiple coders to enhance reliability could be explored. Pilot testing of the interview strategies and overall approach for gathering data suitable for diagramming outlined in Chapter 4 would examine the effectiveness of this approach and enable refinement. It could be tested in various applications, such as qualitative research, implementation science, and program evaluation.

More broadly, the present research can also inform efforts to develop methods for evidence and knowledge synthesis for program development and implementation science. The methods for data collection and analysis of qualitative data in Chapters 3 and 4 can be used for incorporating local stakeholder perspectives and practitioner knowledge, while Chapter 2 can inform the development of causal-loop diagrams as dynamic hypotheses for program theory. An additional source of knowledge to inform diagram development is peer-reviewed scientific evidence. While scientific evidence has informed model development since the beginning of system dynamics and some efforts have been made to establish processes for doing so (Kenzie et al. 2018), systematic, rigorous methods have not yet been established. The methods outlined in 
Chapter 3 could inform such work. Figure 20 illustrates these types of knowledge synthesized in a working dynamic hypothesis. The forms of knowledge and evidence included in the diagram are not exhaustive and constitute a minimum number of perspectives for this approach.

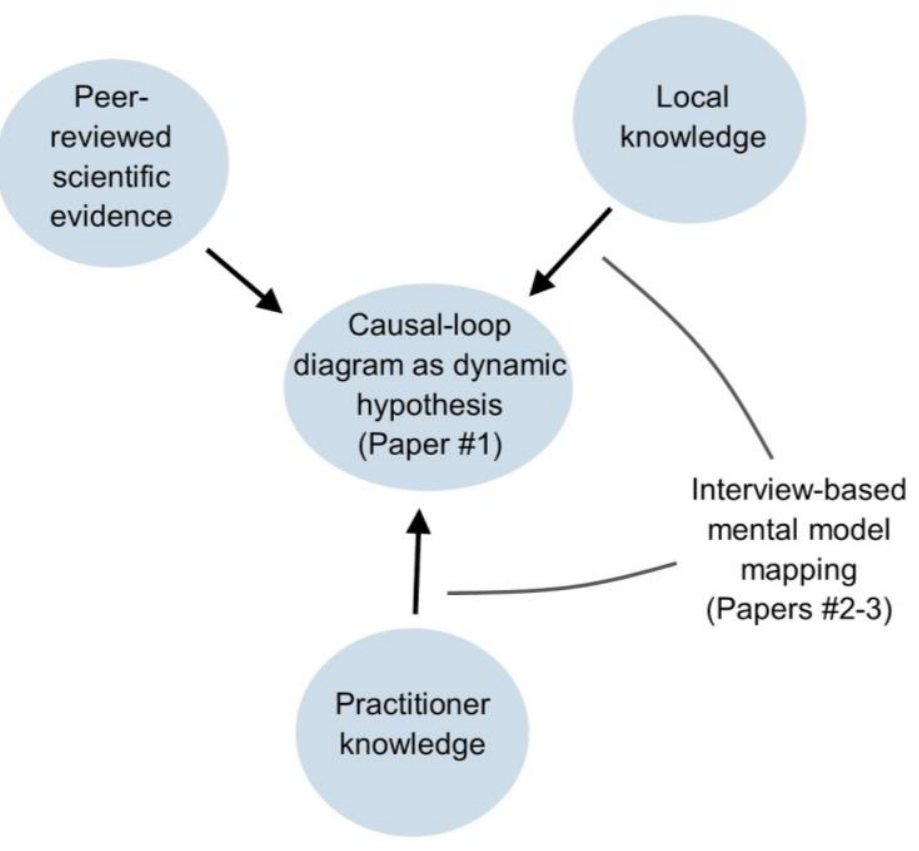

Figure 20. Dissertation papers mapped onto types of knowledge and evidence synthesized to causal-loop diagrams for program development and implementation science.

\subsection{Conclusion}

By advancing methods for developing and analyzing causal-loop diagrams, the present research broadens the potential uses for these methods. Chapter 2 reviews evaluation studies that utilized causal-loop diagrams for program theory and identifies several themes: centering the problem, use of participatory methods, including modeling early in program development, and integration with other methods. Chapter 3 improves upon prior methods for generating causal- 
loop diagrams from qualitative data by using software to increase efficiency, better track sources, and enhance identification of implied causal structures. Chapter 4 incorporates this causal structure analysis approach into an iterative, participatory framework for mapping mental models and provides strategies for designing and conducting interviews suitable for this type of analysis. Together, this research contributes to recognizing the modeler as co-creator, reframing the relationship between intervention and context, and enables more diverse uses for causal-loop diagrams. Further research should further evaluate these methods in various applications, including the synthesis of different forms of knowledge for decision-making.

\subsection{Postscript}

Forrester, the creator of system dynamics, had the following critique of causalloop diagramming (Forrester 2007):

Those who take the road of systems thinking and causal loop diagrams are not practicing system dynamics. They remain dependent on the human mind for solving the dynamic behaviors. It has been repeatedly demonstrated that the human mind is not suited for solving high-order dynamic feedback systems. Such simplifications of system dynamics will almost always lack clarity, lack insight, fail to show how the problems at hand are being caused, and incorrectly evaluate and compare alternative future policies. We should not be surprised that audiences show indifference. Only by going the full road to extensive computer simulations is one prepared for the depth of understanding required in real-world situations.

It is true that the human mind is largely incapable of anticipating the behavior associated with nonlinear causal structures. But Forrester is making a key 
assumption—-that dynamic feedback systems are something to be solved. In complex sociotechnical systems, especially "wicked" ones in which stakeholders disagree about basic problem definitions, what constitutes a "solution"? When simulation models consist largely of estimated parameters and equations, as is common for such systems, how truly useful are those outputs? Simulation models of social systems have the veneer of precision and quantification, but they are crafted through numerous subjective decisions about what to include or exclude (problem definition), how to set up the model (dynamic hypothesis), how to quantify relationships and initial conditions (parameters), and which interventions to examine (model use). Creating and playing around with these arguably qualitative models can be a powerful way to examine the strength of one's own assumptions, as long as those assumptions stay out in the open and guide how the models are used.

A key strength of causal-loop diagrams, on the other hand, is that they allow people to "get their models out there" without being hampered by having to specify equations. Mental models can be described with "words or lists or pictures or arrows showing what you think is connected to what," according to Meadows (2008). She continues,

The more you do that, in any form, the clearer your thinking will become, the faster you will admit your uncertainties and correct your mistakes, and the more flexible you will learn to be. ... Getting models out into the light of day, making them as rigorous as possible, testing them against the evidence, and being willing to scuttle them if they are no longer supported is nothing more than practicing the scientific method. 
But what broader good is served by making mental models visible? Meadows (2008) left us with this advice:

People who are raised in the industrial world and who get enthused about systems thinking are likely to make a terrible mistake. They are likely to assume that here, in systems analysis, in interconnection and complication, in the power of the computer, here at last, is the key to prediction and control. This mistake is likely because the mind-set of the industrial world assumes that there is a key to prediction and control. . . . [But] social systems are the external manifestations of cultural thinking patterns and of profound human needs, emotions, strengths, and weaknesses. ... We can't control systems or figure them out. But we can dance with them! . . Living successfully in a world of systems requires more of us than our ability to calculate. It requires our full humanity-our rationality, our ability to sort out truth from falsehood, our intuition, our compassion, our vision, and our morality.

By expanding and adding rigor to methods for representing our shared or individual mental models, I hope my research enables us to get closer to Meadows' vision for a world that is "envisioned and brought lovingly into being" (2008). 


\section{References}

Abdelbari, Hassan, and Kamran Shafi. 2017. "A Computational IntelligenceBased Method to 'Learn' Causal Loop Diagram-like Structures from Observed Data." System Dynamics Review 33 (1): 3-33. https://doi.org/10.1002/sdr.1567.

Alibage, Ahmed A. n.d. "Achieving High Reliability Organizations Using Fuzzy Cognitive Maps - the Case of Offshore Oil and Gas," 360.

Alibage, Ahmed, Antonie J Jetter, Payam Aminpour, Steven A Gray, and Steven Scyphers. 2018. "Exploratory Participatory Modeling with FCM to Overcome Uncertainty: Improving Safety Culture in Oil and Gas Operations," 9.

Alizadeh, Yasser, and Antonie Jetter. 2017. "Content Analysis Using Fuzzy Cognitive Map (FCM): A Guide to Capturing Causal Relationships from Secondary Sources of Data." In 1-11. IEEE. https://doi.org/10.23919/PICMET.2017.8125305.

Alonge, O., S. Lin, T. Igusa, and D. H. Peters. 2017. "Improving Health Systems Performance in Low- and Middle-Income Countries: A System Dynamics Model of the Pay-for-Performance Initiative in Afghanistan." Health Policy and Planning 32 (10): 1417-26. https://doi.org/10.1093/heapol/czx122.

Anderson, Virginia, and Lauren Johnson. 1997. Systems Thinking Basics. Cambridge, MA: Pegasus Communications.

Aubrecht, Katherine B., Yehudit Judy Dori, Thomas A. Holme, Rea Lavi, Stephen A. Matlin, MaryKay Orgill, and Heather Skaza-Acosta. 2019. "Graphical Tools for Conceptualizing Systems Thinking in Chemistry Education." Journal of Chemical Education 96 (12): 2888-2900. https://doi.org/10.1021/acs.jchemed.9b00314.

Bala, Bilash Kanti, Fatimah Mohamed Arshad, and Kusairi Mohd Noh. 2017. "Causal Loop Diagrams." In System Dynamics, 37-51. Springer Texts in Business and Economics. Springer, Singapore. https://doi.org/10.1007/978-981-10-2045-2_3.

Baskerville, N. B., C. Liddy, and W. Hogg. 2012. "Systematic Review and MetaAnalysis of Practice Facilitation Within Primary Care Settings." The Annals of Family Medicine 10 (1): 63-74. https://doi.org/10.1370/afm.1312.

Birks, Melanie, Ysanne Chapman, and Karen Francis. 2008. "Memoing in Qualitative Research: Probing Data and Processes." Journal of Research in Nursing 13 (1): 68-75. https://doi.org/10.1177/1744987107081254.

Biroscak, Brian. 2014. "Use of System Dynamics Modeling to Explicate the Theory-of-Change of a Social Marketing Innovation." Graduate Theses and Dissertations, July. https://scholarcommons.usf.edu/etd/5184.

Biroscak, Brian, Tali Schneider, Anthony D. Panzera, Carol A. Bryant, Robert J. McDermott, Alyssa B. Mayer, Mahmooda Khaliq, et al. 2014. "Applying Systems Science to Evaluate a Community-Based Social Marketing Innovation: A Case Study." Social Marketing Quarterly 20 (4): 247-67. https://doi.org/10.1177/1524500414556649.

Braun, Virginia, and Victoria Clarke. 2006. "Using Thematic Analysis in 
Psychology." Qualitative Research in Psychology 3 (2): 77-101. https://doi.org/10.1191/1478088706qp063oa.

Brennan, Laura K., Nasim S. Sabounchi, Allison L. Kemner, and Peter Hovmand. 2015. "Systems Thinking in 49 Communities Related to Healthy Eating, Active Living, and Childhood Obesity." Journal of Public Health Management and Practice 21: S55-69.

Britt, Heather, and M Patsalides. 2013. "Complexity-Aware Monitoring." Discussion Note, Monitoring and Evaluation Series.

Burke, Daniel D. 2006. "System Dynamics-Based Computer Simulations and Evaluation." In Systems Concepts in Evaluation: An Expert Anthology, 4759. American Evaluation Association Press.

Byng, Richard, Ian Norman, and Sally Redfern. 2005. "Using Realistic Evaluation to Evaluate a Practice-Level Intervention to Improve Primary Healthcare for Patients with Long-Term Mental Illness." Evaluation 11 (1): 69-93. https://doi.org/10.1177/1356389005053198.

Cabrera, Derek, Laura Colosi, and Claire Lobdell. 2008. "Systems Thinking." Evaluation and Program Planning 31 (3): 299-310. https://doi.org/10.1016/j.evalprogplan.2007.12.001.

Chen, Huey T. 1990. Theory-Driven Evaluations. SAGE.

Clark, Helene. 2012. "Intervention Logic and Theories of Change: Qhat Are They, How to Build Them, How to Use Them." ActKnowledge.

Cohen, Deborah J., Benjamin F. Crabtree, Rebecca S. Etz, Bijal A.

Balasubramanian, Katrina E. Donahue, Laura C. Leviton, Elizabeth C.

Clark, Nicole F. Isaacson, Kurt C. Stange, and Lawrence W. Green. 2008. "Fidelity versus Flexibility: Translating Evidence-Based Research into Practice." American Journal of Preventive Medicine 35 (5 Suppl): S381389. https://doi.org/10.1016/j.amepre.2008.08.005.

Coyle, Geoff. 2004. Practical Strategy: Structured Tools and Techniques. 1st edition. Harlow: Pearson College Div.

Crabtree, Benjamin F., Benjamin F. Crabtree, and William L. Miller. 1999. Doing Qualitative Research. SAGE.

Creswell, John W., and Johanna Creswell Báez. 2020. 30 Essential Skills for the Qualitative Researcher. SAGE Publications.

Cruz, Cindy. 2008. "Notes on Immigration, Youth, and Ethnographic Silence." Theory Into Practice 47 (1): 67-73. https://doi.org/10.1080/00405840701764797.

Damschroder, Laura J., David C. Aron, Rosalind E. Keith, Susan R. Kirsh, Jeffery A. Alexander, and Julie C. Lowery. 2009. "Fostering Implementation of Health Services Research Findings into Practice: A Consolidated Framework for Advancing Implementation Science." Implementation Science 4 (1): 50. https://doi.org/10.1186/1748-5908-4-50.

Doan, Son, Elly W. Yang, Sameer S. Tilak, Peter W. Li, Daniel S. Zisook, and Manabu Torii. 2019. "Extracting Health-Related Causality from Twitter Messages Using Natural Language Processing." BMC Medical Informatics and Decision Making 19 (3): 79. https://doi.org/10.1186/s12911-019-0785- 
0.

Douthwaite, Boru, and Elizabeth Hoffecker. 2017. "Towards a Complexity-Aware Theory of Change for Participatory Research Programs Working within Agricultural Innovation Systems." Agricultural Systems 155 (Supplement C): 88-102. https://doi.org/10.1016/j.agsy.2017.04.002.

Douthwaite, Boru, John Mayne, Cynthia McDougall, and Rodrigo PazYbarnegaray. 2017. "Evaluating Complex Interventions: A Theory-Driven Realist-Informed Approach." Evaluation 23 (3): 294-311. https://doi.org/10.1177/1356389017714382.

Doyle, James K. 1997. "The Cognitive Psychology of Systems Thinking." System Dynamics Review 13 (3): 253-65. https://doi.org/10.1002/(SICI)10991727(199723)13:3<253::AID-SDR129>3.0.CO;2-H.

Doyle, James K., and David N. Ford. 1999. "Mental Models Concepts Revisited: Some Clarifications and a Reply to Lane." System Dynamics Review 15 (4): 411-15. https://doi.org/10.1002/(SICI)1099-

1727(199924)15:4<411::AID-SDR181>3.0.CO;2-R.

Duboz, Raphaël, Pierre Echaubard, Panomsak Promburom, Margaret Kilvington, Helen Ross, Will Allen, John Ward, Guillaume Deffuant, Michel de GarineWichatitsky, and Aurélie Binot. 2018. "Systems Thinking in Practice: Participatory Modeling as a Foundation for Integrated Approaches to Health." Frontiers in Veterinary Science 5. https://doi.org/10.3389/fvets.2018.00303.

Dyehouse, Melissa, Deborah Bennett, Jon Harbor, Amy Childress, and Melissa Dark. 2009. "A Comparison of Linear and Systems Thinking Approaches for Program Evaluation Illustrated Using the Indiana Interdisciplinary GK12." Evaluation and Program Planning 32 (3): 187-96.

https://doi.org/10.1016/j.evalprogplan.2009.03.001.

Ebenso, Bassey, Ana Manzano, Benjamin Uzochukwu, Enyi Etiaba, Reinhard Huss, Tim Ensor, James Newell, et al. 2019. "Dealing with Context in Logic Model Development: Reflections from a Realist Evaluation of a Community Health Worker Programme in Nigeria." Evaluation and Program Planning 73 (April): 97-110. https://doi.org/10.1016/j.evalprogplan.2018.12.002.

Eker, Sibel, and Nici Zimmermann. 2016. "Using Textual Data in System

Dynamics Model Conceptualization." Systems 4 (4): 28.

https://doi.org/10.3390/systems4030028.

Ezzy, Douglas. 2013. Qualitative Analysis. Routledge.

Flynn, Rachel, Thomas Rotter, Dawn Hartfield, Amanda S. Newton, and Shannon D. Scott. 2019. "A Realist Evaluation to Identify Contexts and Mechanisms That Enabled and Hindered Implementation and Had an Effect on Sustainability of a Lean Intervention in Pediatric Healthcare." BMC Health Services Research 19 (1): 912. https://doi.org/10.1186/s12913-019-4744-3.

Ford, Andrew. 2010. Modeling the Environment, Second Edition. Island Press. Forrester, Jay W. 1993. "System Dynamics and the Lessons of 35 Years." In A 
Systems-Based Approach to Policymaking, edited by Kenyon B. De Greene, 199-240. Boston, MA: Springer US. https://doi.org/10.1007/9781-4615-3226-2 7.

2007. "System Dynamics—the next Fifty Years." System Dynamics Review 23 (2-3): 359-70. https://doi.org/10.1002/sdr.381.

Forss, Kim, Mita Marra, and Robert Schwartz. 2011. Evaluating the Complex: Attribution, Contribution, and Beyond. Transaction Publishers.

Fredericks, Kimberly A., Michael Deegan, and Joanne G. Carman. 2008. "Using System Dynamics as an Evaluation Tool: Experience From a Demonstration Program." American Journal of Evaluation 29 (3): 251-67. https://doi.org/10.1177/1098214008319446.

Funnell, Sue C., and Patricia J. Rogers. 2011. Purposeful Program Theory: Effective Use of Theories of Change and Logic Models. 1 edition. San Francisco, CA: Jossey-Bass.

Gates, Emily F. 2016. "Making Sense of the Emerging Conversation in Evaluation about Systems Thinking and Complexity Science." Evaluation and Program Planning 59 (December): 62-73. https://doi.org/10.1016/j.evalprogplan.2016.08.004. . 2017. "Learning from Seasoned Evaluators: Implications of Systems Approaches for Evaluation Practice." Evaluation 23 (2): 152-71. https://doi.org/10.1177/1356389017697613.

Grammatikopoulos, Vasilis. 2012. "Integrating Program Theory and SystemsBased Procedures in Program Evaluation: A Dynamic Approach to Evaluate Educational Programs." Educational Research and Evaluation 18 (1): 53-64. https://doi.org/10.1080/13803611.2011.640874.

Green, Bart N., Claire D. Johnson, and Alan Adams. 2006. "Writing Narrative Literature Reviews for Peer-Reviewed Journals: Secrets of the Trade." Journal of Chiropractic Medicine 5 (3): 101-17. https://doi.org/10.1016/S0899-3467(07)60142-6.

Grice, Herbert. 1975. "Logic and Conversation." In Speech Acts. Brill.

Grizzle, Gloria A., and Carole D. Pettijohn. 2002. "Implementing PerformanceBased Program Budgeting: A System-Dynamics Perspective." Public Administration Review 62 (1): 51-62. https://doi.org/10.1111/15406210.00154.

Grove, John T. 2015. "Aiming for Utility in 'Systems-Based Evaluation': A Research-Based Framework for Practitioners." IDS Bulletin 46 (1): 58-70.

Gubrium, Jaber, and James Holstein. 2001. Handbook of Interview Research. 2455 Teller Road, Thousand Oaks California 91320 United States of America: SAGE Publications, Inc. https://doi.org/10.4135/9781412973588. Gudykunst, William B., Stella Ting-Toomey, and Elizabeth Chua. 1988. Culture and Interpersonal Communication. Culture and Interpersonal Communication. Thousand Oaks, CA, US: Sage Publications, Inc.

Hanin, Marie C. E., Kevin Queenan, Sara Savic, Esron Karimuribo, Simon R. Rüegg, and Barbara Häsler. 2018. "A One Health Evaluation of the Southern African Centre for Infectious Disease Surveillance." Frontiers in 
Veterinary Science 5. https://doi.org/10.3389/fvets.2018.00033.

Harachi, Tracy W., Robert D. Abbott, Richard F. Catalano, Kevin P. Haggerty, and Charles B. Fleming. 1999. "Opening the Black Box: Using Process Evaluation Measures to Assess Implementation and Theory Building." American Journal of Community Psychology 27 (5): 711-31. https://doi.org/10.1023/A:1022194005511.

Hassmiller Lich, Kristen, Jennifer Brown Urban, Leah Frerichs, and Gaurav Dave. 2017. "Extending Systems Thinking in Planning and Evaluation Using Group Concept Mapping and System Dynamics to Tackle Complex Problems." Evaluation and Program Planning 60 (Supplement C): 254-64. https://doi.org/10.1016/j.evalprogplan.2016.10.008.

Hawe, Penelope. 2015. "Lessons from Complex Interventions to Improve Health." Annual Review of Public Health 36 (1): 307-23. https://doi.org/10.1146/annurev-publhealth-031912-114421.

Head, Brian W., and John Alford. 2015. "Wicked Problems: Implications for Public Policy and Management." Administration \& Society 47 (6): 711-39. https://doi.org/10.1177/0095399713481601.

Hebbard, Claire E. 2010. "SYSTEMS EVALUATION PROTOCOL."

Homer, Jack B., and Gary B. Hirsch. 2006. "System Dynamics Modeling for Public Health: Background and Opportunities." American Journal of Public Health 96 (3): 452-58. https://doi.org/10.2105/AJPH.2005.062059.

Homer, Jack, Bobby Milstein, Darwin Labarthe, Diane Orenstein, Kristina Wile, Justin Trogdon, and Philip Huang. 2009. "Simulating and Evaluating Local Interventions to Improve Cardiovascular Health." Preventing Chronic Disease 7 (1). https://www.ncbi.nlm.nih.gov/pmc/articles/PMC2811513/.

Houston, Danielle, Bernadette Wright, and Steven E. Wallis. 2017. "ReStructuring Evaluation Findings into Useful Knowledge." Journal of MultiDisciplinary Evaluation 13 (29): 31-41.

Hovmand, Peter. 2014. Community Based System Dynamics. New York: Springer-Verlag. //www.springer.com/us/book/9781461487623.

Hovmand, Peter S. 2014. "Group Model Building and Community-Based System Dynamics Process." In Community Based System Dynamics, 17-30. New York, NY: Springer New York. https://doi.org/10.1007/978-1-4614-876302 .

Hovmand Peter S., Andersen David F., Rouwette Etiënne, Richardson George P., Rux Krista, and Calhoun Annaliese. 2012. "Group Model- Building 'Scripts' as a Collaborative Planning Tool." Systems Research and Behavioral Science 29 (2): 179-93. https://doi.org/10.1002/sres.2105. Innovation Network. n.d. "Logic Model Workbook." Innovation Network. https://www.innonet.org/media/logic_model_workbook_0.pdf.

Jetter, Antonie J., and Kasper Kok. 2014. "Fuzzy Cognitive Maps for Futures Studies-A Methodological Assessment of Concepts and Methods." Futures 61 (September): 45-57. https://doi.org/10.1016/j.futures.2014.05.002. Jones, Natalie D., Tarek Azzam, Dana Linnell Wanzer, Darrel Skousen, Ciara 
Knight, and Nina Sabarre. 2019. "Enhancing the Effectiveness of Logic Models." American Journal of Evaluation, April, 1098214018824417. https://doi.org/10.1177/1098214018824417.

Jones, N. A, H. Ross, T. Lynam, P. Perez, and A. Leitch. 2011. "Mental Models: An Interdisciplinary Synthesis of Theory and Methods." Ecology and Society 16 (1): 46.

Jung, Jae Un. 2017. "Reducing Subjectivity in the System Dynamics Modeling Process: An Interdisciplinary Approach." In Intelligent Data Engineering and Automated Learning - IDEAL 2017, 365-75. Lecture Notes in Computer Science. Springer, Cham. https://doi.org/10.1007/978-3-31968935-7_40.

Kellogg Foundation, W. K. 2004. "Logic Model Development Guide." https://www.bttop.org/sites/default/files/public/W.K.\%20Kellogg\%20LogicM odel.pdf.

Kenzie, Erin S., Elle L. Parks, Erin D. Bigler, David W. Wright, Miranda M. Lim, James C. Chesnutt, Gregory W. J. Hawryluk, Wayne Gordon, and Wayne Wakeland. 2018. "The Dynamics of Concussion: Mapping Pathophysiology, Persistence, and Recovery with Causal-Loop Diagramming." Frontiers in Neurology 9. https://doi.org/10.3389/fneur.2018.00203.

Kim, Daniel H. 1994. Systems Archetypes. Toolbox Reprint Series. Cambridge, Mass: Pegasus Communications.

Kim, Daniel H, and Virginia Anderson. 2007. Systems Archetype Basics: From Story to Structure. Waltham: Pegasus Communications.

Kim, Hyunjung, and David F. Andersen. 2012. "Building Confidence in Causal Maps Generated from Purposive Text Data: Mapping Transcripts of the Federal Reserve." System Dynamics Review 28 (4): 311-28. https://doi.org/10.1002/sdr.1480.

Knai, Cécile, Mark Petticrew, Nick Douglas, Mary Alison Durand, Elizabeth Eastmure, Ellen Nolte, and Nicholas Mays. 2018. "The Public Health Responsibility Deal: Using a Systems-Level Analysis to Understand the Lack of Impact on Alcohol, Food, Physical Activity, and Workplace Health Sub-Systems." International Journal of Environmental Research and Public Health 15 (12): 2895. https://doi.org/10.3390/ijerph15122895.

Kvale, Steinar, and Svend Brinkmann. 2009. InterViews: Learning the Craft of Qualitative Research Interviewing, 2nd Ed. InterViews: Learning the Craft of Qualitative Research Interviewing, 2nd Ed. Thousand Oaks, CA, US: Sage Publications, Inc.

Kwamie, Aku, Han van Dijk, and Irene Akua Agyepong. 2014. "Advancing the Application of Systems Thinking in Health: Realist Evaluation of the Leadership Development Programme for District Manager DecisionMaking in Ghana." Health Research Policy and Systems 12 (1): 29. https://doi.org/10.1186/1478-4505-12-29.

Lane, David C. 2008. "The Emergence and Use of Diagramming in System Dynamics: A Critical Account." Systems Research and Behavioral Science 
25 (1): 3-23. https://doi.org/10.1002/sres.826.

Lee, Bruce, and et. al. 2016. "SPACES MERL: Systems and Complexity White Paper." Washington, DC: US Agency for International Development. http://globalknowledgeinitiative.org/wp-content/uploads/2016/09/Systemsand-Complexity-White-Paper_USAID_GKI_LINC_JHU_RAN-March2016.pdf.

Léger, Anaïs, Katharina D. C. Stärk, Jonathan Rushton, and Liza R. Nielsen. 2018. "A One Health Evaluation of the University of Copenhagen Research Centre for Control of Antibiotic Resistance." Frontiers in Veterinary Science 5. https://doi.org/10.3389/fvets.2018.00194.

Luna-Reyes, Luis Felipe, and Deborah Lines Andersen. 2003. "Collecting and Analyzing Qualitative Data for System Dynamics: Methods and Models." System Dynamics Review 19 (4): 271-96. https://doi.org/10.1002/sdr.280.

Manzano, Ana. 2016. "The Craft of Interviewing in Realist Evaluation." Evaluation 22 (3): 342-60. https://doi.org/10.1177/1356389016638615.

Martinez- Moyano, Ignacio J., and George P. Richardson. 2013. "Best Practices in System Dynamics Modeling." System Dynamics Review 29 (2): 102-23. https://doi.org/10.1002/sdr.1495.

Mason, Paul, and Marian Barnes. 2007. "Constructing Theories of Change: Methods and Sources." Evaluation 13 (2): 151-70. https://doi.org/10.1177/1356389007075221.

Mayne, J, and E Stern. 2013. "Impact Evaluation of Natural Resource Management Research Programs: A Broader View." Series Report No. 84. Canberra: Australian Centre for International Agricultural Research. https://aciar.gov.au/publication/technical-publications/impact-evaluationnatural-resource-management-research-programs-broader-view.

McDermott, Tom, Molly Nadolski, and Lindsey Sheppard. 2015. "Use of Systemigrams to Identify Emergence in Complex Adaptive Systems." INCOSE International Symposium 25 (1): 582-99. https://doi.org/10.1002/j.2334-5837.2015.00083.x.

Meadows, Donella H. 2008. Thinking in Systems: A Primer. White River Junction, Vt: Chelsea Green Publishing.

Meinherz, Franziska, Paola Espín, and Robinson Salazar. 2016. "When Stakeholders Cannot Be Involved in a Modelling Process: Integrating Participatory Modelling and Consultative Approaches." Proceedings of the 34th International Conference of the System Dynamics Society, Delft, Netherlands -- July 17-21, 2016. 2016. https://infoscience.epfl.ch/record/226382.

Mendoza, Guillermo A., and Ravi Prabhu. 2006. "Participatory Modeling and Analysis for Sustainable Forest Management: Overview of Soft System Dynamics Models and Applications." Forest Policy and Economics 9 (2): 179-96. https://doi.org/10.1016/j.forpol.2005.06.006.

Merrill, J. A., M. Deegan, R. V. Wilson, R. Kaushal, and K. Fredericks. 2013. "A System Dynamics Evaluation Model: Implementation of Health Information Exchange for Public Health Reporting." Journal of the American Medical 
Informatics Association 20 (e1): e131-38. https://doi.org/10.1136/amiajnl2012-001289.

Mierlo, Barbara van, Cees Leeuwis, Ruud Smits, and Rosalinde Klein Woolthuis. 2010. "Learning towards System Innovation: Evaluating a Systemic Instrument." Technological Forecasting and Social Change 77 (2): 31834. https://doi.org/10.1016/j.techfore.2009.08.004.

Miller, Robin Lin. 2013. "Logic Models: A Useful Way to Study Theories of Evaluation Practice?" Evaluation and Program Planning, Using Logic Models to Facilitate Comparisons of Evaluation Theory, 38 (June): 77-80. https://doi.org/10.1016/j.evalprogplan.2012.03.019.

Mitchell, Melanie. 2009. Complexity: A Guided Tour. Oxford University Press. Mobus, George E., and Michael C. Kalton. 2015. Principles of Systems Science. Understanding Complex Systems. New York: Springer-Verlag. https://doi.org/10.1007/978-1-4939-1920-8.

Morrison, Diane M., Marilyn J. Hoppe, Mary Rogers Gillmore, Carisa Kluver, Darrel Higa, and Elizabeth A. Wells. 2009. "Replicating An Intervention: The Tension Between Fidelity and Adaptation." AIDS Education and Prevention 21 (2): 128-40. https://doi.org/10.1521/aeap.2009.21.2.128.

Mowles, Chris. 2014. "Complex, but Not Quite Complex Enough: The Turn to the Complexity Sciences in Evaluation Scholarship." Evaluation 20 (2): 160175.

Mukumbang, Ferdinand C, Bruno Marchal, Sara Van Belle, and Brian van Wyk. 2019. "Using the Realist Interview Approach to Maintain Theoretical Awareness in Realist Studies." Qualitative Research, October, 1468794119881985. https://doi.org/10.1177/1468794119881985.

Muñoz-Prieto, Alberto, Liza R. Nielsen, Silvia Martinez-Subiela, Jovita Mazeikiene, Pia Lopez-Jornet, Sara Savić, and Asta Tvarijonaviciute. 2018. "Application of the NEOH Framework for Self-Evaluation of One Health Elements of a Case-Study on Obesity in European Dogs and DogOwners." Frontiers in Veterinary Science 5. https://doi.org/10.3389/fvets.2018.00163.

Munro, Eileen. 2010. "The Munro Review of Child Protection Part One: A Systems Analysis." London. https://assets.publishing.service.gov.uk/government/uploads/system/uploa ds/attachment_data/file/624949/TheMunroReview-Part_one.pdf.

Mutale, Wilbroad, Helen Ayles, Virginia Bond, Namwinga Chintu, Roma Chilengi, Margaret Tembo Mwanamwenge, Angela Taylor, Neil Spicer, and Dina Balabanova. 2017. "Application of Systems Thinking: 12- month Postintervention Evaluation of a Complex Health System Intervention in Z Ambia: The Case of the BHOMA." Journal of Evaluation in Clinical Practice 23 (2): 439-52. https://doi.org/10.1111/jep.12354.

Mutale, Wilbroad, Dina Balabanova, Namwinga Chintu, Margaret Tembo Mwanamwenge, and Helen Ayles. 2016. "Application of System Thinking Concepts in Health System Strengthening in Low-Income Settings: A Proposed Conceptual Framework for the Evaluation of a Complex Health 
System Intervention: The Case of the BHOMA Intervention in Zambia." Journal of Evaluation in Clinical Practice 22 (1): 112-21.

https://doi.org/10.1111/jep.12160.

Nelson, Gayle L., Mahmoud Al Batal, and Waguida El Bakary. 2002. "Directness vs. Indirectness: Egyptian Arabic and US English Communication Style." International Journal of Intercultural Relations 26 (1): 39-57. https://doi.org/10.1016/S0147-1767(01)00037-2.

Newcomer, K, Harry Hatry, and Joseph Wholey. 2015. Handbook of Practical Program Evaluation. John Wiley \& Sons.

Nickerson, Raymond S. 1998. "Confirmation Bias: A Ubiquitous Phenomenon in Many Guises." Review of General Psychology 2 (2): 175-220. https://doi.org/10.1037/1089-2680.2.2.175.

O'Connell, D, N Abel, N Grigg, Y Maru, J Butler, A Cowie, Samantha StoneJovicich, et al. 2016. "Designing Projects in a Rapidly Changing World: Guidelines for Embedding Resilience, Adaptation and Transformation into Sustainable Development Projects (Version 1.0)." Washington, DC: Global Environment Facility. https://thegef.org/sites/default/files/publications/STAP-RaptaGuidelines2016.pdf.

Okumu, Moses, Hiroo Kato, and Nishesh Chalise. 2016. "A System Dynamics Approach to Process Evaluation of Pen-Based Digital Media-Making Projects." In Revolutionizing Education with Digital Ink, 209-21. HumanComputer Interaction Series. Springer, Cham. https://doi.org/10.1007/9783-319-31193-7_14.

Olabisi, Laura Schmitt. 2013. "Participatory Modeling in Environmental Systems." In Proceedings of the 31st. International Conference of the System Dynamics Society.

https://proceedings.systemdynamics.org/2013/proceed/papers/P1295.pdf.

Ostrom, E. 2009. "A General Framework for Analyzing Sustainability of SocialEcological Systems." Science 325 (5939): 419-22.

https://doi.org/10.1126/science.1172133.

Owen, Brynle, Andrew D. Brown, Jill Kuhlberg, Lynne Millar, Melanie Nichols, Christina Economos, and Steven Allender. 2018. "Understanding a Successful Obesity Prevention Initiative in Children under 5 from a Systems Perspective." PLOS ONE 13 (3): e0195141.

https://doi.org/10.1371/journal.pone.0195141.

Özesmi, Uygar, and Stacy L. Özesmi. 2004. "Ecological Models Based on People's Knowledge: A Multi-Step Fuzzy Cognitive Mapping Approach.” J. of Ecological Modelling, 3-64.

Parsons, Beverly A. 2007. "The State of Methods and Tools for Social Systems Change." American Journal of Community Psychology 39 (3-4): 405-9. https://doi.org/10.1007/s10464-007-9118-z.

Patton, Michael Quinn. 2010. Developmental Evaluation: Applying Complexity Concepts to Enhance Innovation and Use. Guilford Press.

Pawson, Ray. 1996. "Theorizing the Interview." The British Journal of Sociology 
47 (2): 295. https://doi.org/10.2307/591728.

Pawson, Ray, and Nicholas Tilley. 1997. Realistic Evaluation. SAGE.

Paz-Ybarnegaray, Rodrigo, and Boru Douthwaite. 2017. "Outcome Evidencing: A Method for Enabling and Evaluating Program Intervention in Complex Systems." American Journal of Evaluation 38 (2): 275-93. https://doi.org/10.1177/1098214016676573.

Pruyt, Erik. 2006. "What Is System Dynamics? A Paradigmatic Inquiry." In Proceedings of the 2006 Conference of the System Dynamics Society, 29. Nijmegen: System Dynamics Society.

Renger, Ralph, Lewe Atkinson, Jessica Renger, Jirina Renger, and Gary Hart. 2019. "The Connection between Logic Models and Systems Thinking Concepts." Evaluation Journal of Australasia 19 (2): 79-87. https://doi.org/10.1177/1035719X19853660.

Renmans, Dimitri, Nathalie Holvoet, and Bart Criel. 2017. "Combining TheoryDriven Evaluation and Causal Loop Diagramming for Opening the 'Black Box' of an Intervention in the Health Sector: A Case of PerformanceBased Financing in Western Uganda." International Journal of Environmental Research and Public Health 14 (9): 1007. https://doi.org/10.3390/ijerph14091007. . 2020. "No Mechanism Without Context: Strengthening the Analysis of Context in Realist Evaluations Using Causal Loop Diagramming." New Directions for Evaluation 2020 (167): 101-14. https://doi.org/10.1002/ev.20424.

Reynolds, Martin, Emily Gates, Richard Hummelbrunner, Mita Marra, and Bob Williams. 2016. "Towards Systemic Evaluation." Systems Research and Behavioral Science 33 (5): 662-73. https://doi.org/10.1002/sres.2423.

Richardson, George P. 1986. "Problems with Causal-Loop Diagrams." System Dynamics Review 2 (2): 158-70. https://doi.org/10.1002/sdr.4260020207.

Richardson, George P., and David F. Andersen. 1995. "Teamwork in Group Model Building." System Dynamics Review 11 (2): 113-37. https://doi.org/10.1002/sdr.4260110203.

Richardson, George P., Jac A. M. Vennix, David F. Andersen, John Rohrbaugh, and W. A. Wallace. 1989. "Eliciting Group Knowledge for Model-Building." In Computer-Based Management of Complex Systems, edited by Peter M. Milling and Erich O. K. Zahn, 343-57. Berlin, Heidelberg: Springer. https://doi.org/10.1007/978-3-642-74946-9_36.

Ritchie, Jane, and Jane Lewis. 2003. Qualitative Research Practice: A Guide for Social Science Students and Researchers. SAGE.

Rittel, Horst W. J., and Melvin M. Webber. 1973. "Dilemmas in a General Theory of Planning." Policy Sciences 4 (2): 155-69.

https://doi.org/10.1007/BF01405730.

Rogers, Patricia J. 2008. "Using Programme Theory to Evaluate Complicated and Complex Aspects of Interventions." Evaluation 14 (1): 29-48. https://doi.org/10.1177/1356389007084674.

Rouwette, Etiënne A. J. A., Jac A. M. Vennix, and Theo van Mullekom. 2002. 
"Group Model Building Effectiveness: A Review of Assessment Studies." System Dynamics Review 18 (1): 5-45. https://doi.org/10.1002/sdr.229.

Rüegg, Simon R., Barbara Häsler, and Jakob Zinsstag, eds. 2018. Integrated Approaches to Health: A Handbook for the Evaluation of One Health. The Netherlands: Wageningen Academic Publishers. https://doi.org/10.3920/978-90-8686-875-9.

Rüegg, Simon R., Liza Rosenbaum Nielsen, Sandra C. Buttigieg, Mijalche Santa, Maurizio Aragrande, Massimo Canali, Timothy Ehlinger, et al. 2018. "A Systems Approach to Evaluate One Health Initiatives." Frontiers in Veterinary Science 5. https://doi.org/10.3389/fvets.2018.00023.

Sapsford, Roger, and P Abbott. 1992. Research Into Practice: A Reader for Nurses and the Caring Professions. Open University Press.

Sarriot, Eric, Melanie Morrow, Anne Langston, Jennifer Weiss, Justine Landegger, and Laban Tsuma. 2015. "A Causal Loop Analysis of the Sustainability of Integrated Community Case Management in Rwanda." Social Science \& Medicine 131 (Supplement C): 147-55. https://doi.org/10.1016/j.socscimed.2015.03.014.

Schaffernicht, Martin, and Stefan N. Groesser. 2011. "A Comprehensive Method for Comparing Mental Models of Dynamic Systems." European Journal of Operational Research 210 (1): 57-67. https://doi.org/10.1016/j.ejor.2010.09.003.

Schmitt Olabisi, Laura K., Anne R. Kapuscinski, Kris A. Johnson, Peter B. Reich, Brian Stenquist, and Kathryn J. Draeger. 2010. "Using Scenario Visioning and Participatory System Dynamics Modeling to Investigate the Future: Lessons from Minnesota 2050." Sustainability 2 (8): 2686-2706. https://doi.org/10.3390/su2082686.

Senge, Peter M. 2010. The Fifth Discipline: The Art \& Practice of The Learning Organization. Crown Publishing Group.

Sonawane, S. S., and P. A. Kulkarni. 2014. "Graph Based Representation and Analysis of Text Document: A Survey of Techniques." International Journal of Computer Applications 96 (19): 1-8.

Stame, Nicoletta. 2004. "Theory-Based Evaluation and Types of Complexity." Evaluation 10 (January): 58-76. https://doi.org/10.1177/1356389004043135.

Stave, Krystyna. 2010. "Participatory System Dynamics Modeling for Sustainable Environmental Management: Observations from Four Cases." Sustainability 2 (9): 2762-84. https://doi.org/10.3390/su2092762.

Sterman, John. 2000. Business Dynamics: Systems Thinking and Modeling for a Complex World. Irwin/McGraw-Hill.

Strauss, Anselm L. 1987. Qualitative Analysis for Social Scientists. Cambridge University Press.

Stroh, David Peter. 2015. Systems Thinking For Social Change: A Practical Guide to Solving Complex Problems, Avoiding Unintended Consequences, and Achieving Lasting Results. Chelsea Green Publishing. 
Tàbara, J. David, Elisabet Roca, Cristina Madrid Lopez, Pieter Valkering, Patrik Wallman, and Paul Weaver. 2008. "Integrated Sustainability Assessment of Water Systems: Lessons from the Ebro River Basin." International Journal of Innovation and Sustainable Development - Int J Innovat Sustain Dev 3 (May). https://doi.org/10.1504/IJISD.2008.018193.

Tobin, Rochelle, Jonathan Hallett, Roanna Lobo, and Bruce Richard Maycock. 2019. "Taking a Systems Approach to Explore the Impacts and Outcomes of a Research and Evaluation Capacity Building Partnership: A Protocol." BMJ Open 9 (9): e026706. https://doi.org/10.1136/bmjopen-2018-026706.

Trochim, W, J. B. Urban, Monica Hargraves, Claire E Hebbard, J Buckley, T Archibald, M Johnson, and M Burgermaster. 2016. "The Guide to the Systems Evaluation Protocol (v3.1)." Cornell Office for Reseach on Evaluation.

Turner, Benjamin L., Hyunjung Kim, and David F. Andersen. 2013. "Improving Coding Procedures for Purposive Text Data: Researchable Questions for Qualitative System Dynamics Modeling." System Dynamics Review 29 (4): 253-63. https://doi.org/10.1002/sdr.1506.

Turner, Benjamin L., Melissa Wuellner, Timothy Nichols, and Roger Gates. 2014. "Dueling Land Ethics: Uncovering Agricultural Stakeholder Mental Models to Better Understand Recent Land Use Conversion." Journal of Agricultural and Environmental Ethics 27 (5): 831-56. https://doi.org/10.1007/s10806-014-9494-y.

Valcourt, Nicholas, Jeffrey Walters, Amy Javernick- Will, and Karl Linden. 2020. "Assessing the Efficacy of Group Model Building Workshops in an Applied Setting through Purposive Text Analysis." System Dynamics Review 36 (2): 135-57. https://doi.org/10.1002/sdr.1657.

Vennix, Jac A. M. 1999. "Group Model-Building: Tackling Messy Problems." System Dynamics Review 15 (4): 379-401. https://doi.org/10.1002/(SICI)1099-1727(199924)15:4<379::AIDSDR179>3.0.CO;2-E.

Vennix, Jac A. M., Henk A. Akkermans, and Etiënne A. J. A. Rouwette. 1996. "Group Model-Building to Facilitate Organizational Change: An Exploratory Study." System Dynamics Review 12 (1): 39-58. https://doi.org/10.1002/(SICI)1099-1727(199621)12:1<39::AIDSDR94>3.0.CO;2-K.

Vennix, Jac A. M., and Jan W. Gubbels. 1992. "Knowledge Elicitation in Conceptual Model Building: A Case Study in Modeling a Regional Dutch Health Care System." European Journal of Operational Research, Modelling for Learning, 59 (1): 85-101. https://doi.org/10.1016/03772217(92)90008-W.

Wakeland, Wayne. 2014. "Four Decades of Systems Science Teaching and Research in the USA at Portland State University." Systems 2 (2): 77-88. https://doi.org/10.3390/systems2020077.

Weiss, Carol H. 1997. "Theory-Based Evaluation: Past, Present, and Future." New Directions for Evaluation. 
Weiss, Robert S. 1995. Learning From Strangers: The Art and Method of Qualitative Interview Studies. Simon and Schuster.

Wheat, I. David. 2007. "The Feedback Method of Teaching Macroeconomics: Is It Effective?" System Dynamics Review 23 (4): 391-413. https://doi.org/10.1002/sdr.386.

Wilcox, Bruce A., A. Alonso Aguirre, Nicole De Paula, Boripat Siriaroonrat, and Pierre Echaubard. 2019. "Operationalizing One Health Employing SocialEcological Systems Theory: Lessons From the Greater Mekong SubRegion." Frontiers in Public Health 7. https://doi.org/10.3389/fpubh.2019.00085.

Williams, Bob, and Richard Hummelbrunner. 2010. Systems Concepts in Action: A Practitioner's Toolkit. Stanford University Press.

Williams, Bob, and Iraj Imam, eds. 2007. Systems Concepts in Evaluation: An Expert Anthology. 1st edition. Port Reyes, CA: EdgePress.

Wittenborn, A. K., H. Rahmandad, J. Rick, and N. Hosseinichimeh. 2016. "Depression as a Systemic Syndrome: Mapping the Feedback Loops of Major Depressive Disorder." Psychological Medicine 46 (03): 551-562. https://doi.org/10.1017/S0033291715002044.

Wolf-Branigin, Michael. 2013. Using Complexity Theory for Research and Program Evaluation. Pocket Guides to Social Work Research Methods. Oxford; New York: Oxford University Press.

Wright, Bernadette, and Steven E. Wallis. 2019. Practical Mapping for Applied Research and Program Evaluation. SAGE Publications.

Yearworth, Mike, and Leroy White. 2013. "The Uses of Qualitative Data in Multimethodology: Developing Causal Loop Diagrams during the Coding Process." European Journal of Operational Research 231 (1): 151-61. https://doi.org/10.1016/j.ejor.2013.05.002.

Yus, Francisco. 1999. "Misunderstandings and Explicit/Implicit Communication." Pragmatics 9 (4): 487-517. https://doi.org/10.1075/prag.9.4.01yus.

Zurcher, Kathleen A., Jon Jensen, and Ann Mansfield. 2018. "Using a Systems Approach to Achieve Impact and Sustain Results." Health Promotion Practice 19 (1_suppl): 15S-23S. https://doi.org/10.1177/1524839918784299. 


\section{Appendices}

Appendix A: Examples of Theory of Change diagrams

The following diagrams were presented as examples of the Theory of Change method at www.theoryofchange.org.

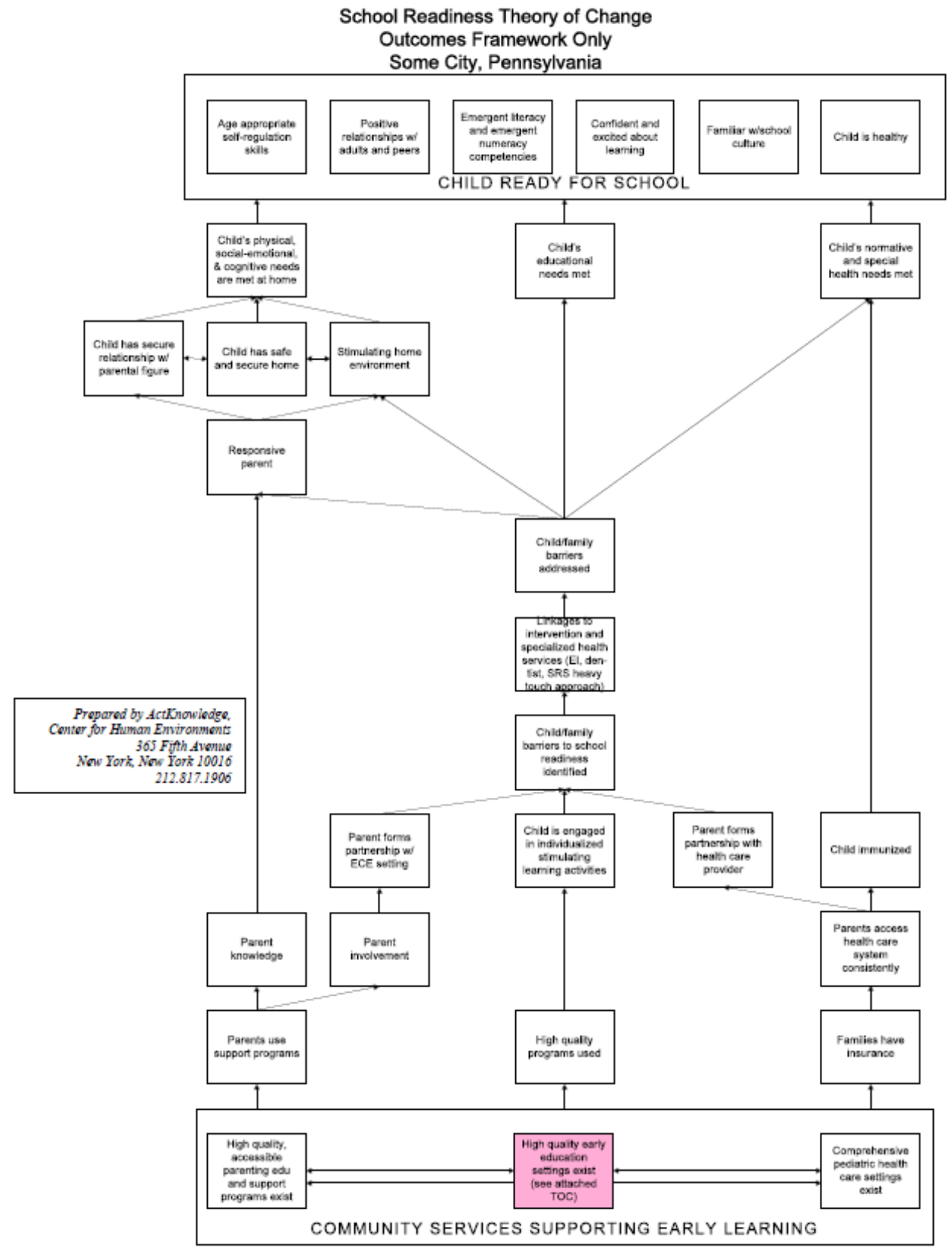




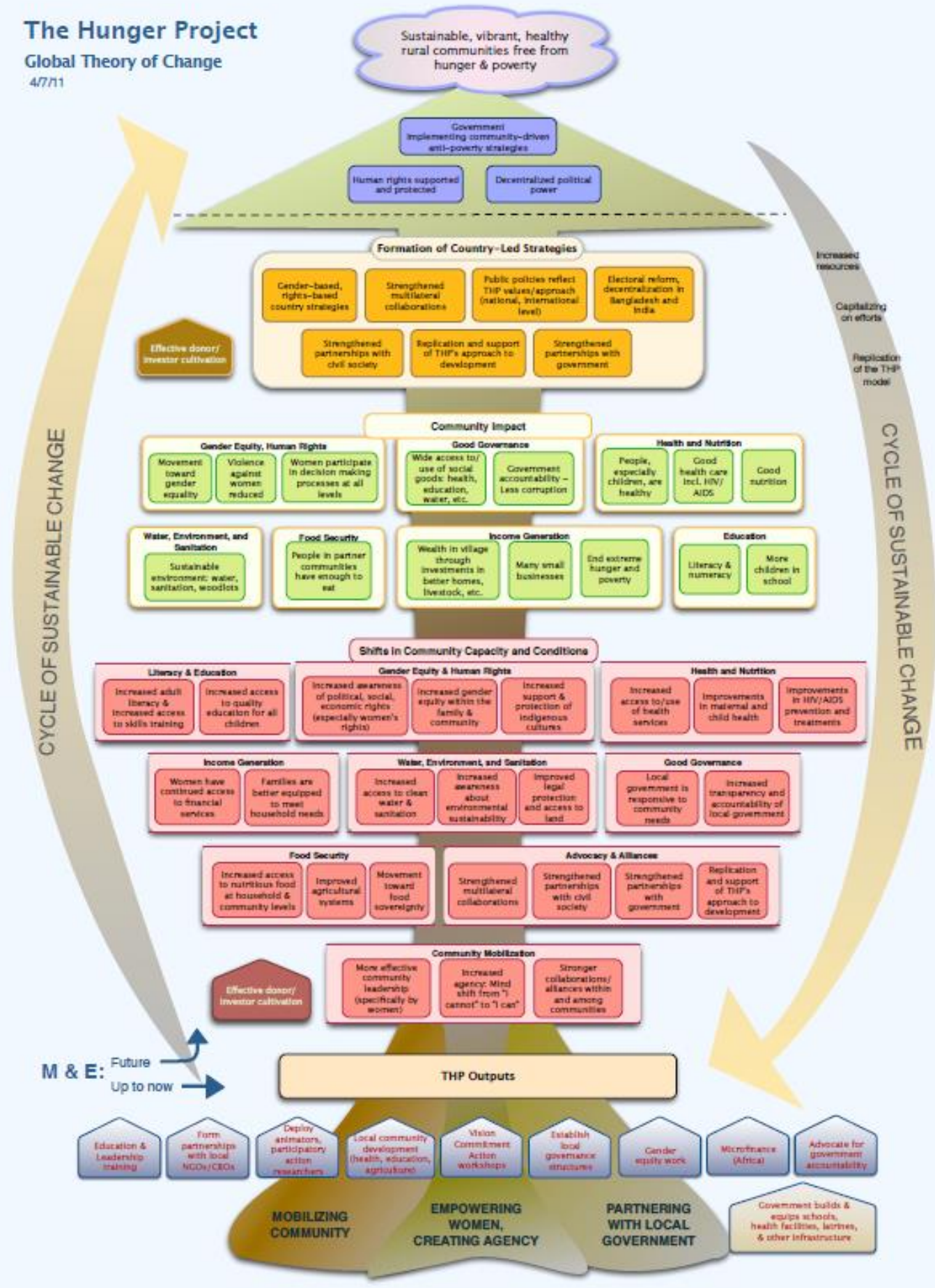




\section{Appendix B: Literature Review Protocol}

\begin{tabular}{|l|l|}
\hline Purpose & $\begin{array}{l}\text { To synthesize findings about the application of causal-loop diagrams to } \\
\text { program theory, particularly prior examples of models used in program } \\
\text { theory and guidelines or advice about the appropriate use of the approach }\end{array}$ \\
\hline Search terms & $\begin{array}{l}\text { "causal-loop diagram" } \\
\text { AND } \\
\text { "program theory" OR "theory of change" OR "theory-based evaluation" } \\
\text { OR "logic model" OR "program evaluation" }\end{array}$ \\
\hline Databases & $\begin{array}{l}\text { Peer-reviewed articles: Google Scholar, PsychINFO, Web of Science, } \\
\text { ERIC, PubMed, PAIS Index, Academic Search Premier } \\
\text { Gray literature: Google, betterevaluation.org, USAID, WHO }\end{array}$ \\
\hline Inclusion criteria & \begin{tabular}{l} 
Sources included in the sample fit all of the following criteria: \\
Published 2000 or later \\
Used causal-loop diagrams or stock-and-flow models \\
The diagram was used for the purpose of describing how a \\
program or intervention was thought to create change (i.e, for \\
program theory) \\
$\begin{array}{l}\text { The diagram was used as part of an evaluation } \\
\text { The publication included a description of the methods used to } \\
\text { create the diagram and an assessment of its effectiveness }\end{array}$ \\
\hline Review process
\end{tabular} \mid $\begin{array}{l}\text { Sources were identified using two methods: } \\
\text { Search results from databases using defined search terms } \\
\text { Snowball sampling from sources cited in identified literature }\end{array}$ \\
\hline
\end{tabular}


Appendix C: Sample of causal-loop diagrams from studies included in review Below is a selection of causal-loop diagrams from the included studies showing diversity in diagram format and scope.

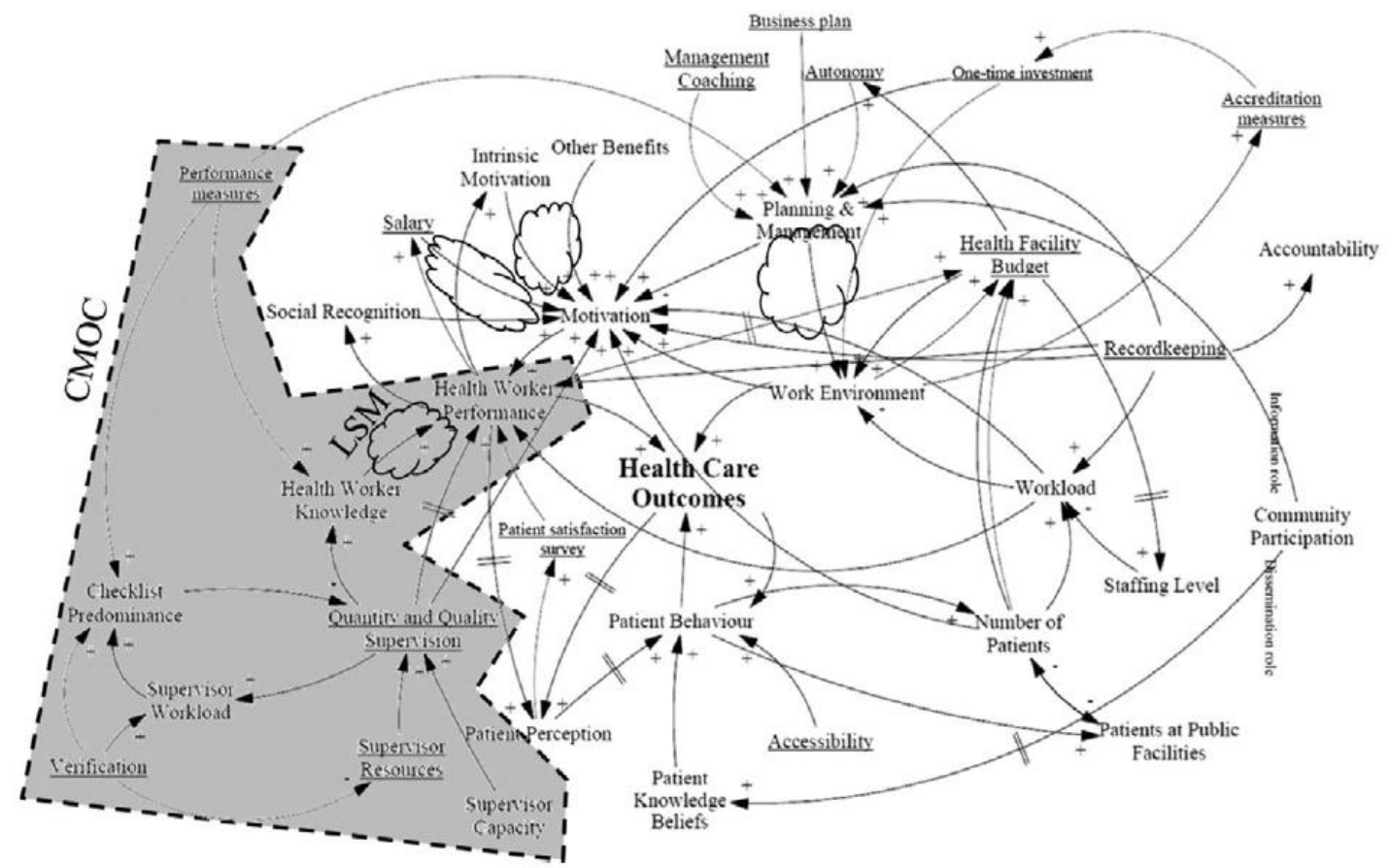

From Renmans et al. 2020

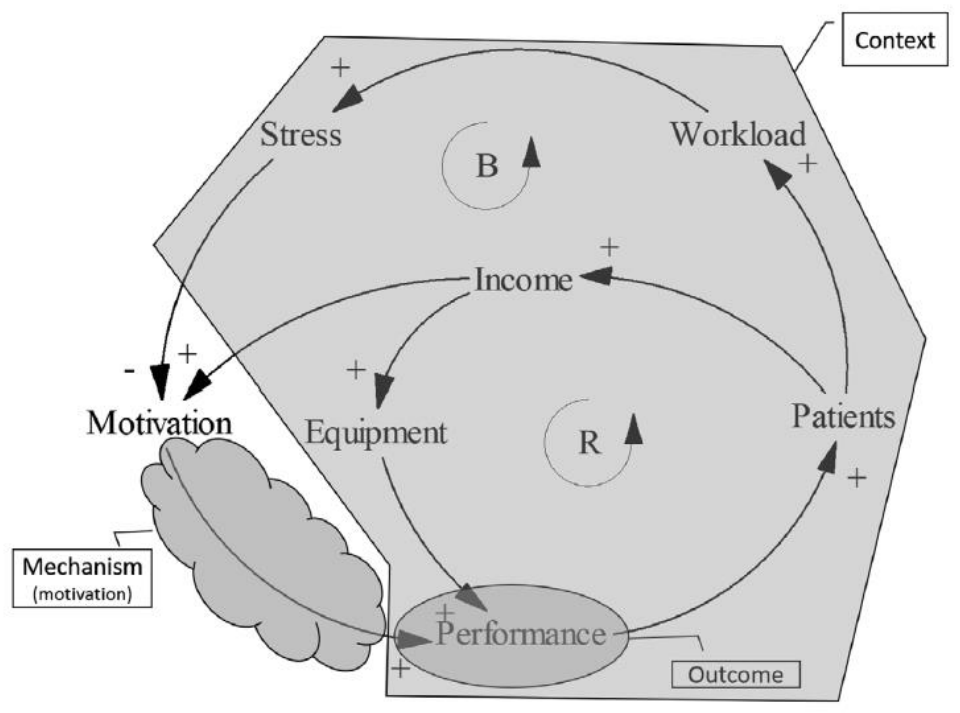

From Renmans et al. 2020 


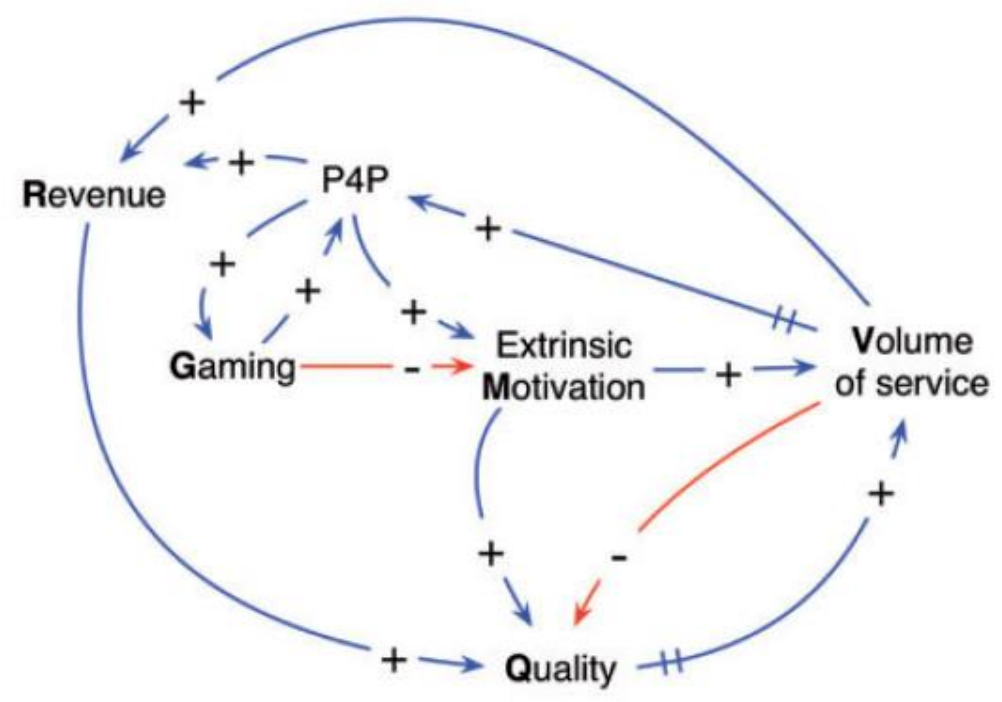

From Alonge et al. 2017

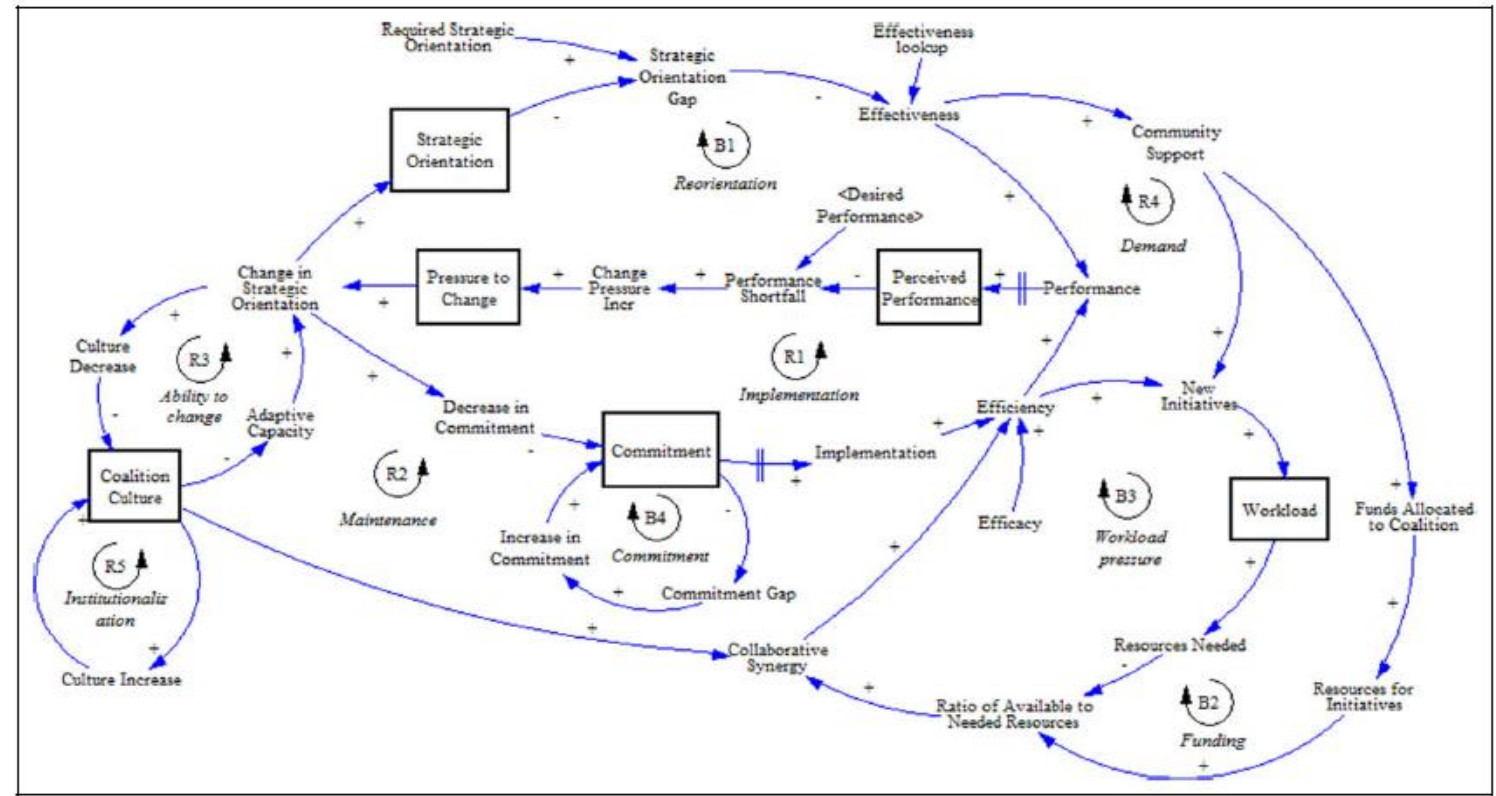

From Biroscak et al. 2014 


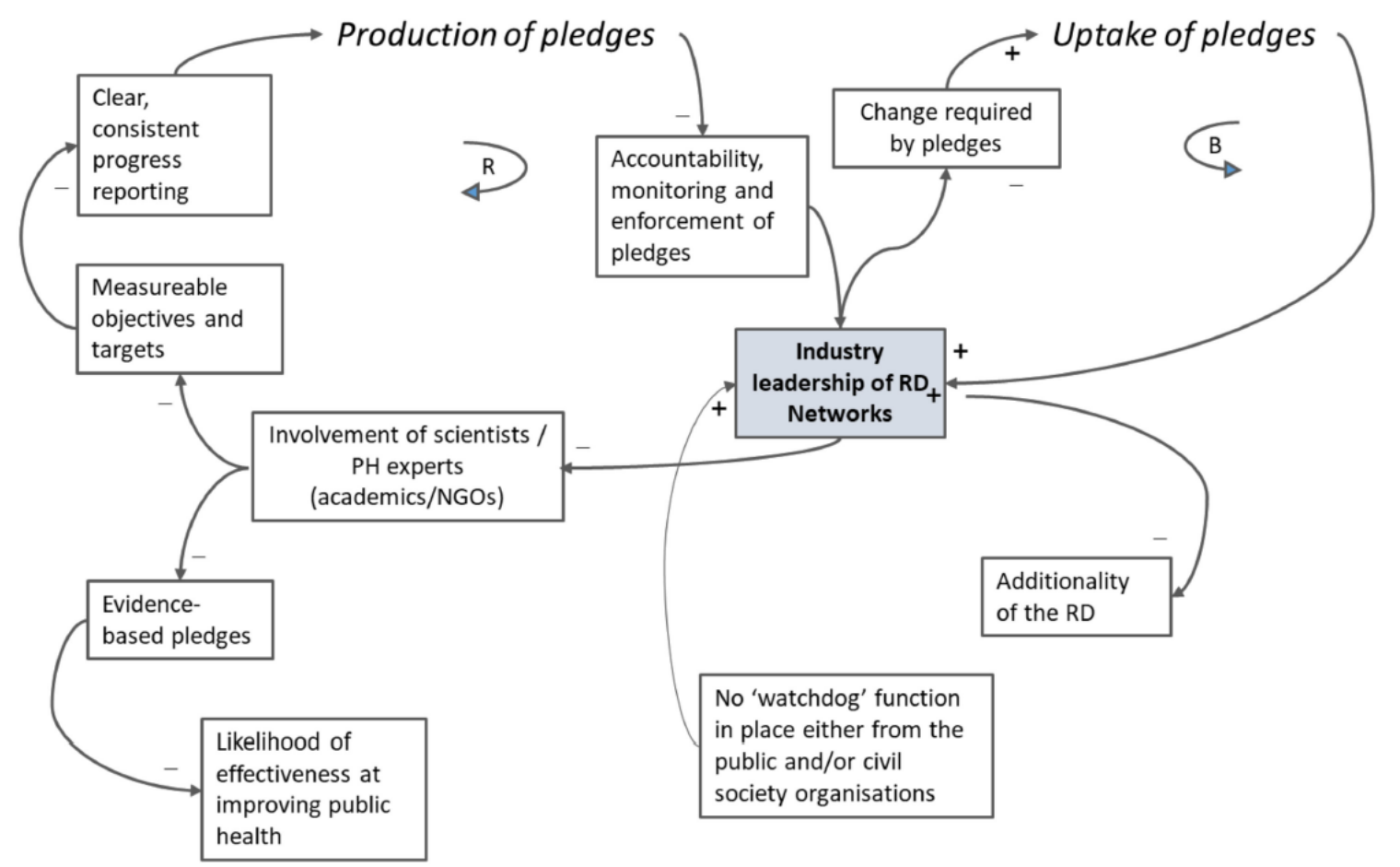

From Knai et al. 2018

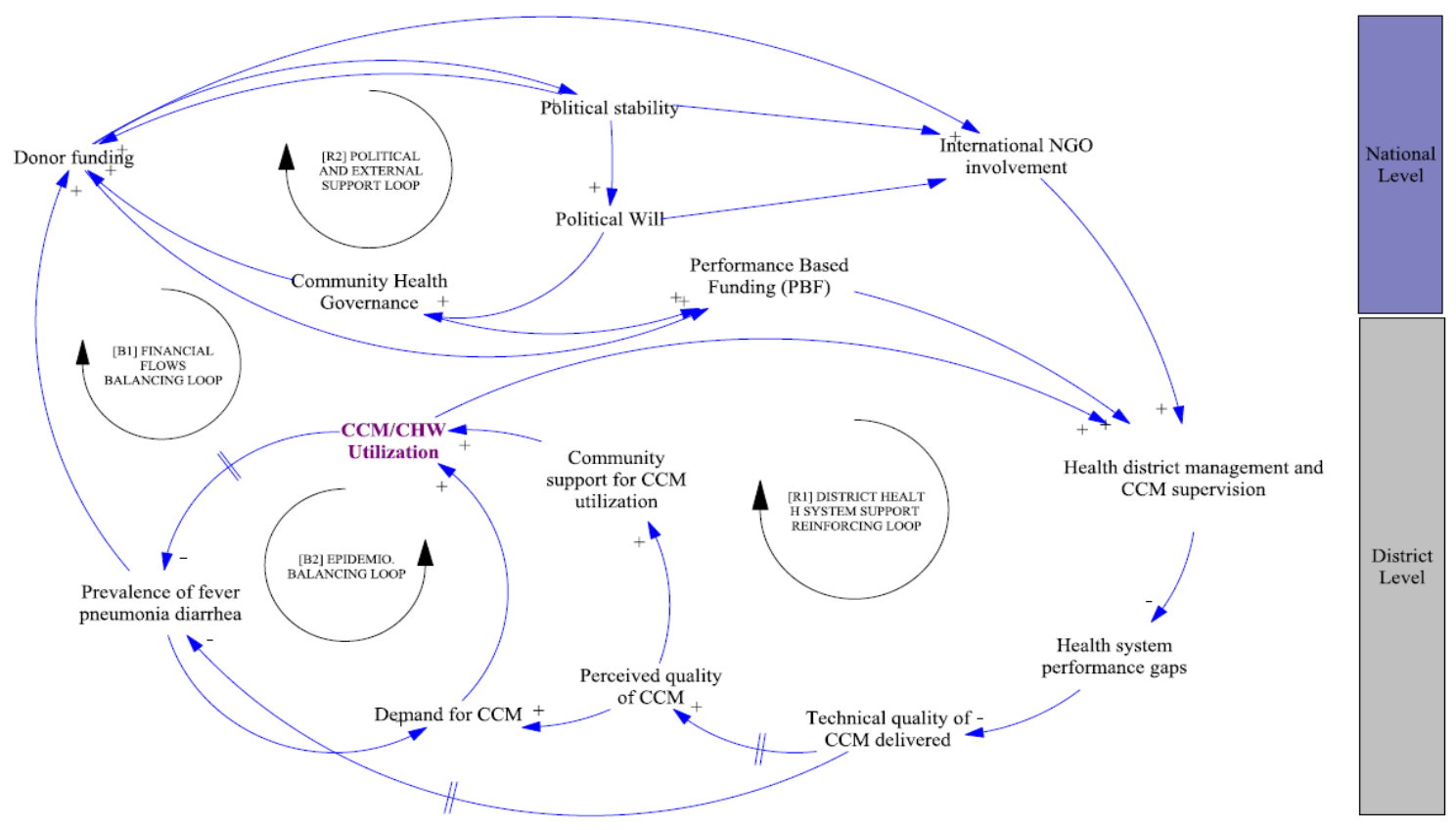


From Sarriot et al. 2015

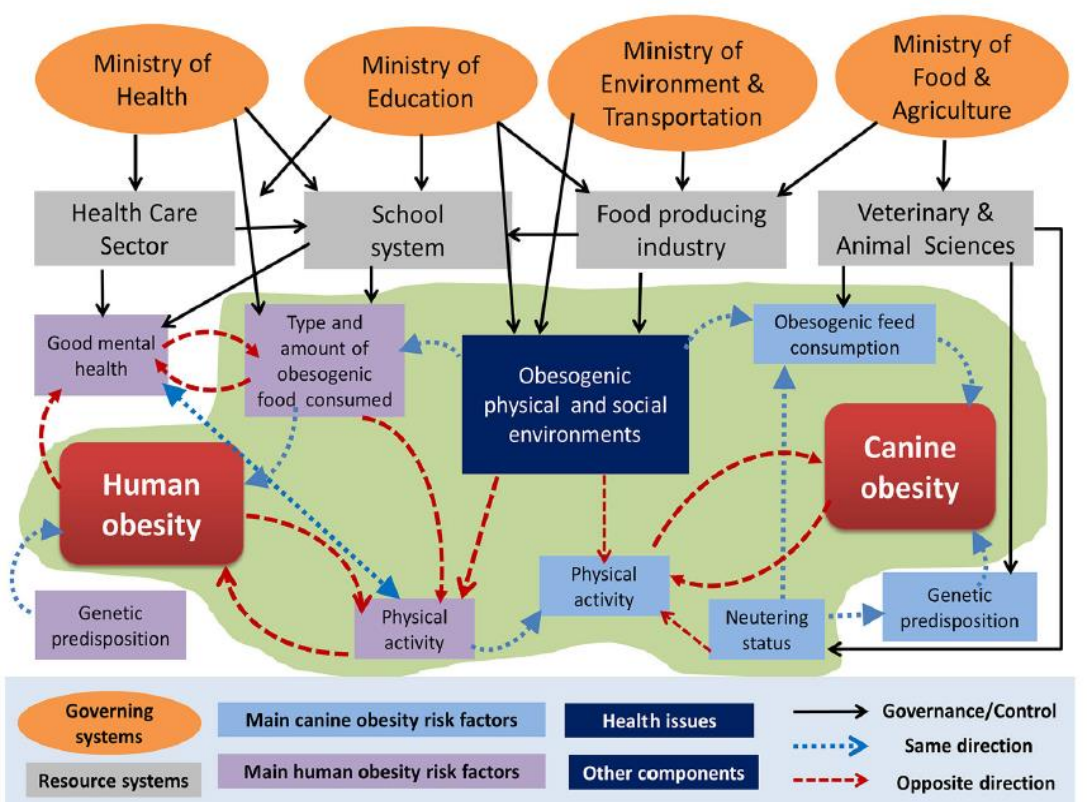

FIGURE 1 | Visual representation of the context of human and pet-dog obesity including linkages and feedback loops in the system. The list of ministries and resource units is not exhaustive and the names are examples as these vary between countries as well as over time within countries. A green shaded area covers the elements considered in the initiative under evaluation.

\section{From Muñoz-Prieto et al. 2018}

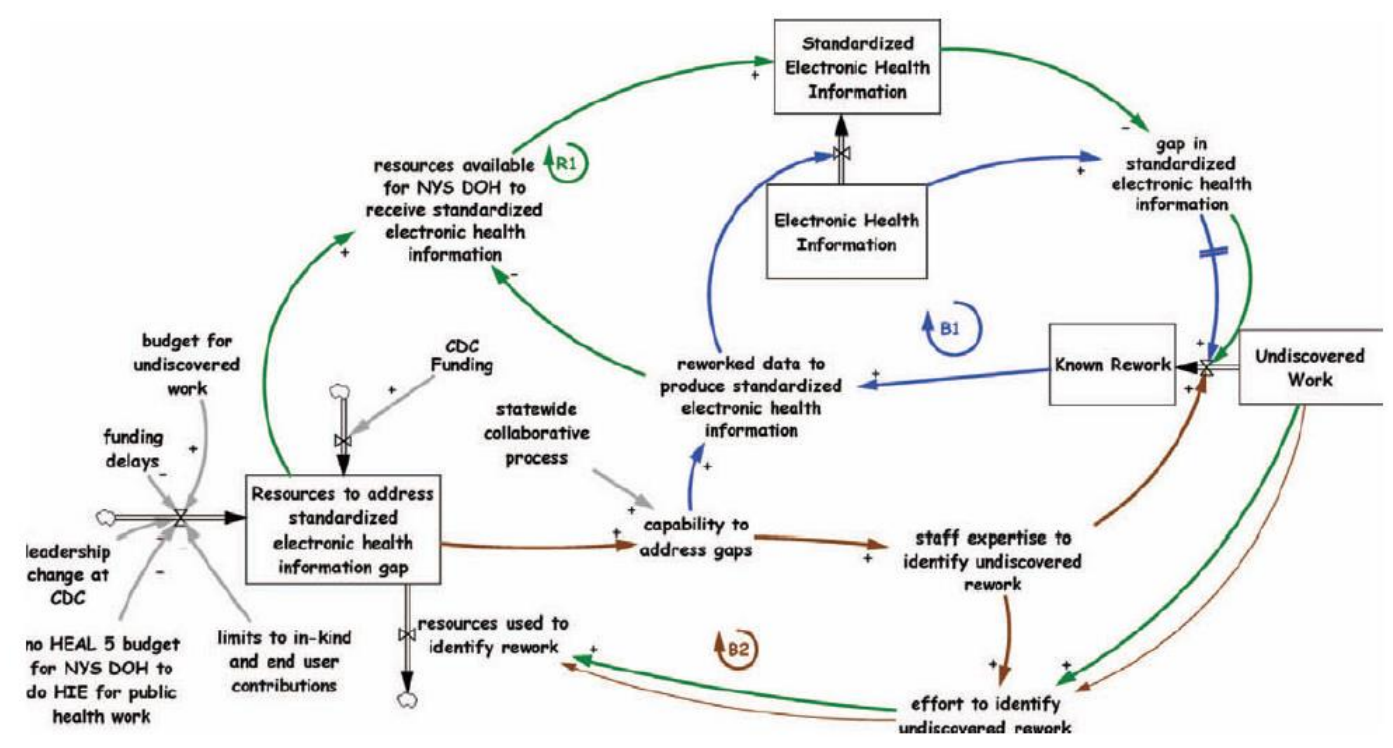

From Merrill et al. 2013 


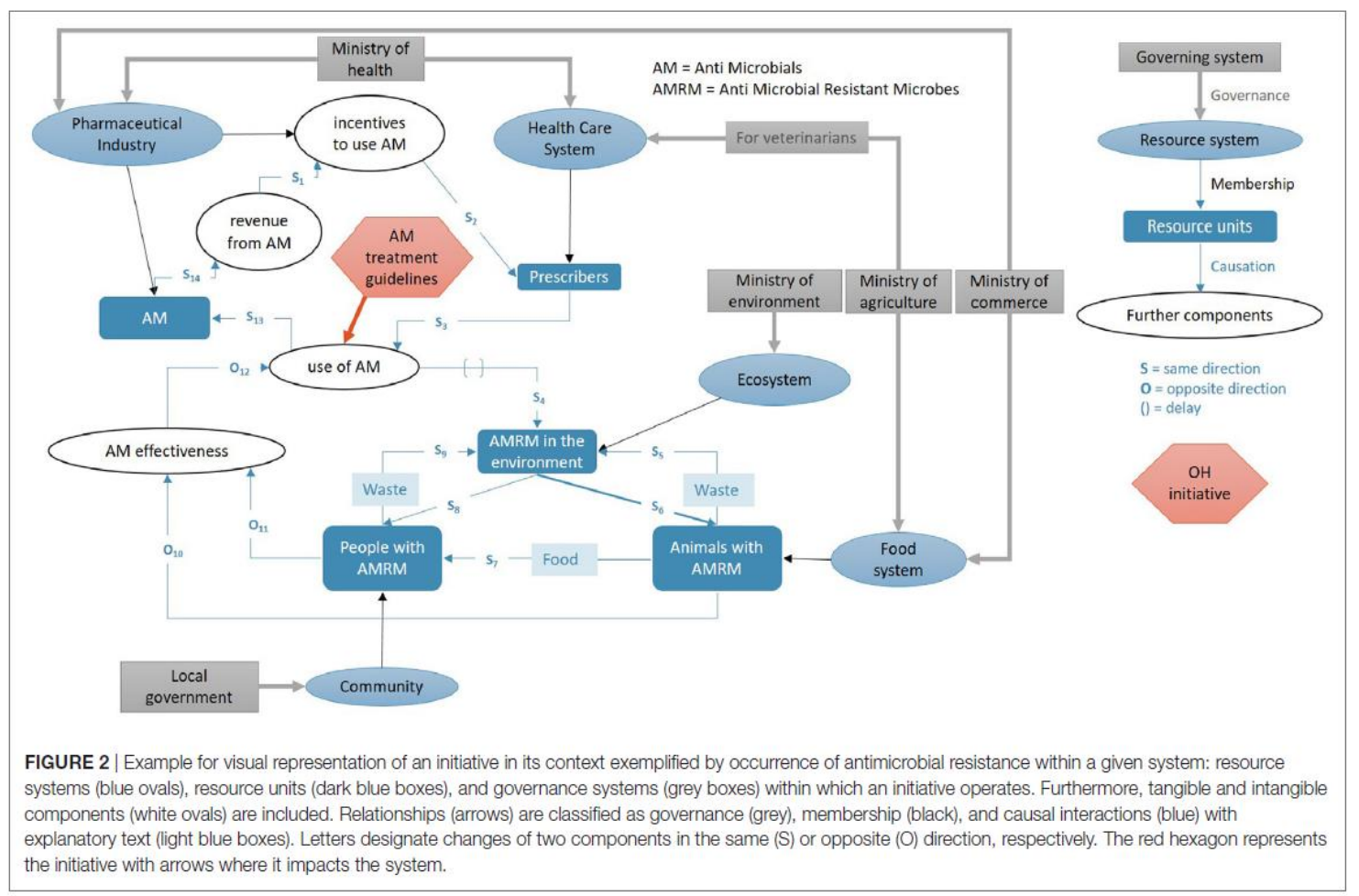

\section{From Rüegg et al. 2018}

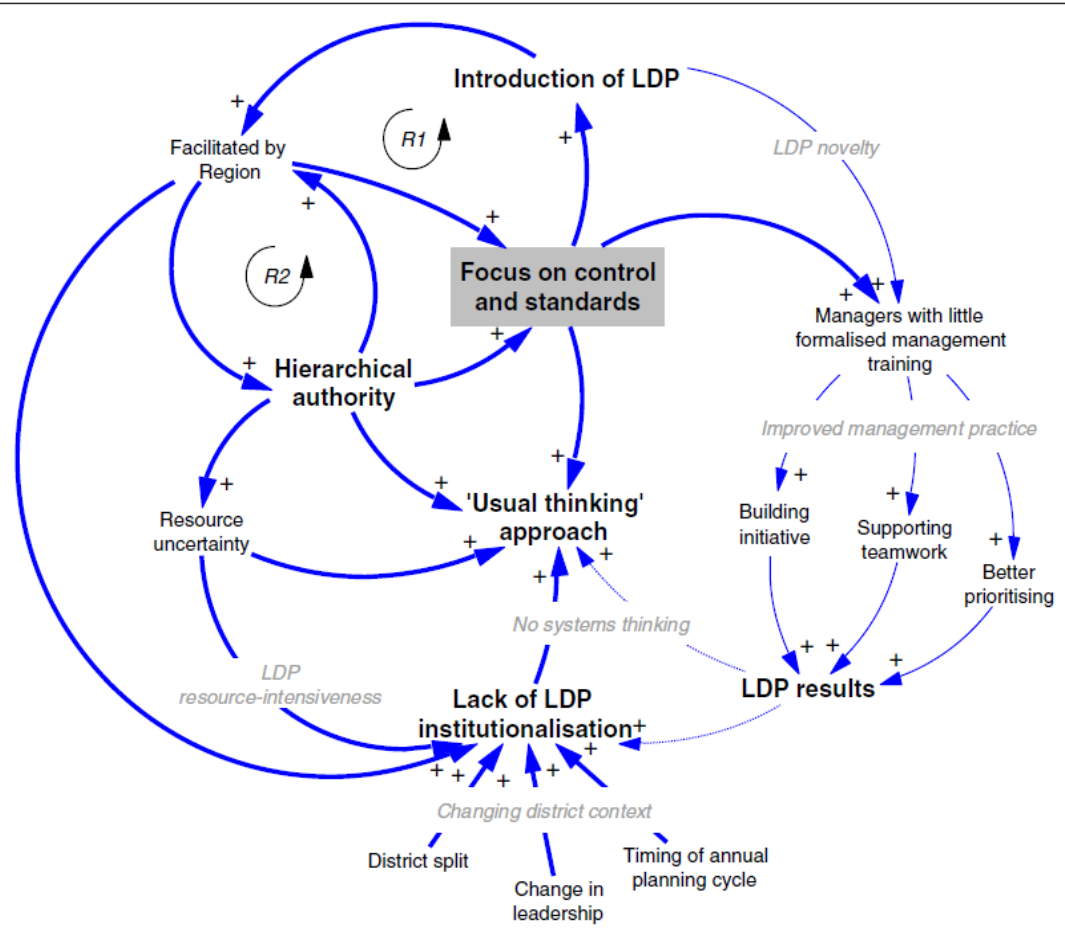

Figure 2 Causal loop diagram of LDP implementation, February to August 2012.

From Kwamie et al. 2014 


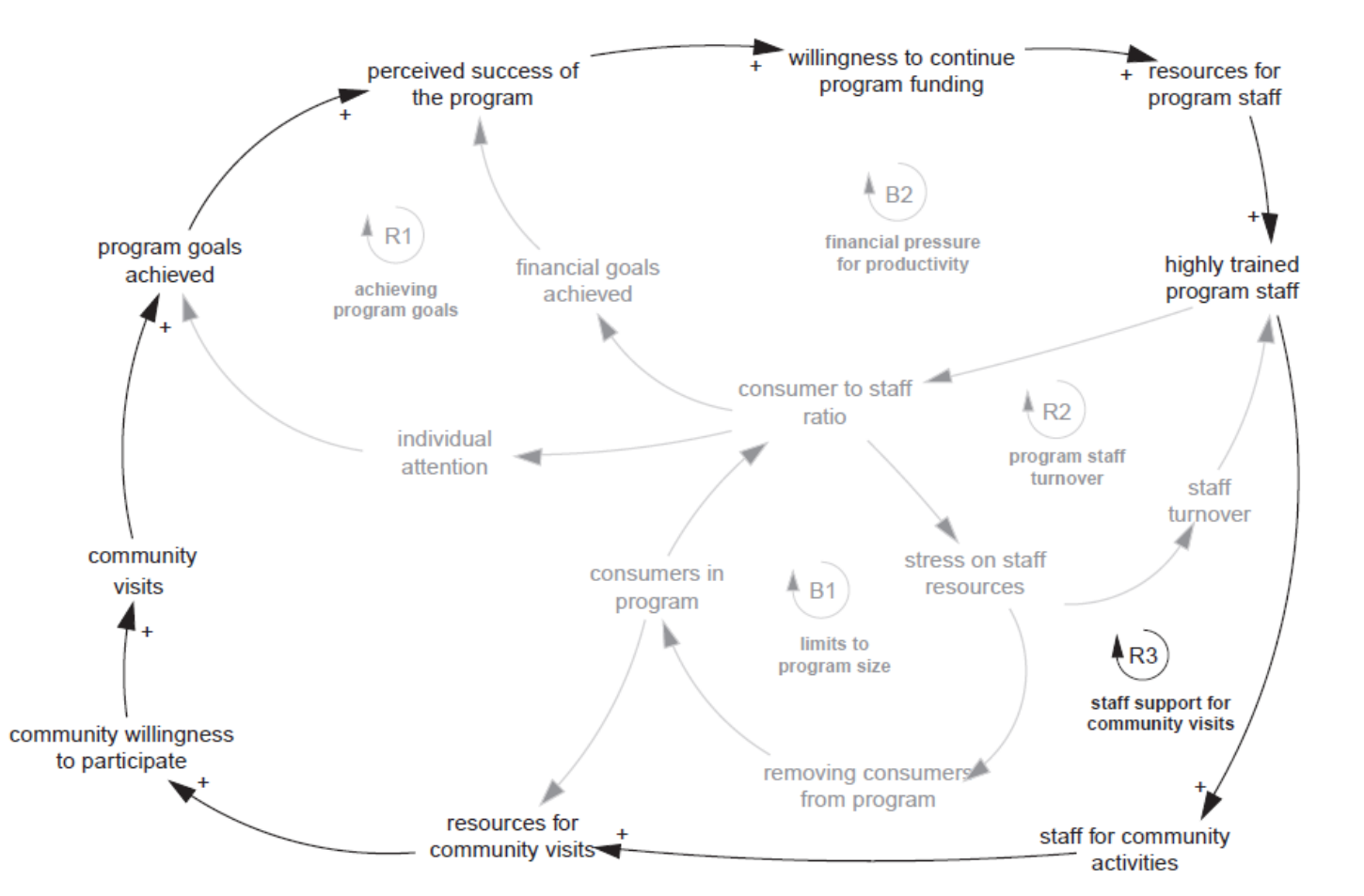

From Fredericks et al. 2008 


\section{Appendix D: Casual mapping table sample}

Below are two tables comprising the causal mapping table for Participant 5.

\begin{tabular}{|c|c|c|}
\hline Label & Description & Tags \\
\hline Ability to get meetings with clinics & $5: 10$ & Link | Context: local \\
\hline BI with patients & $5: 9$ & Loop | Implementation \& intervention \\
\hline $\begin{array}{l}\text { C/S ability to work together for } \\
\text { ANTECEDENT }\end{array}$ & $5: 8$ & Link | Context: local \\
\hline C/S buy-in & $5: 6 ; 5: 9 ; 5: 7 ; 5: 47$ & Link | Clinician \& staff |Count:4 | Loop \\
\hline $\mathrm{C} / \mathrm{S}$ communication within team & $5: 8$ & Link | Context: local \\
\hline $\mathrm{C} / \mathrm{S}$ ego & $5: 8$ & Link | Clinician \& staff \\
\hline C/S knowledge about project & $5: 7$ & Link | Clinician \& staff \\
\hline $\begin{array}{l}\text { C/S perception of improvement } \\
\text { need }\end{array}$ & $5: 17$ & Link | Context: local \\
\hline $\mathrm{C} / \mathrm{S}$ perception of project value & $5: 19$ & Link | Context: local \\
\hline $\mathrm{C} / \mathrm{S}$ receptiveness to $\mathrm{PERC}$ & $5: 17$ & Link | Clinician \& staff | Implied \\
\hline $\begin{array}{l}\mathrm{C} / \mathrm{S} \text { reluctance to meddling from } \\
\text { outsiders from Portland }\end{array}$ & $5: 17$ & Link | Clinician \& staff \\
\hline $\mathrm{C} / \mathrm{S}$ resistance to change & $5: 2 ; 5: 8$ & Link | Clinician \& staff | Count:2 \\
\hline $\mathrm{C} / \mathrm{S}$ satisfaction with project & $\begin{array}{l}5: 19 ; \text { indicator of } \\
\text { clinic success }\end{array}$ & Variable | Indicator | Clinician \& staff \\
\hline $\begin{array}{l}\text { C/S see impact of project on } \\
\text { patients }\end{array}$ & $5: 9$ & Loop | Implementation \& intervention \\
\hline $\mathrm{C} / \mathrm{S}$ trust within team & $5: 8$ & Link | Context: local \\
\hline $\begin{array}{l}\mathrm{C} / \mathrm{S} \text { understanding what to expect } \\
\text { in project }\end{array}$ & $5: 7$ & Link | Clinician \& staff \\
\hline $\begin{array}{l}\mathrm{C} / \mathrm{S} \text { willingness to assess } \\
\text { progress }\end{array}$ & $\begin{array}{l}5: 19 \text {; indicator of } \\
\text { clinic success }\end{array}$ & Variable | Indicator | Clinician \& staff \\
\hline $\begin{array}{l}\text { C/S willingness to look at SBIRT } \\
\text { outcomes }\end{array}$ & $\begin{array}{l}5: 19 ; \text { indicator of } \\
\text { clinic success }\end{array}$ & Variable | Indicator | Clinician \& staff \\
\hline $\begin{array}{l}\text { C/S willingness to make changes } \\
\text { w/SBIRT }\end{array}$ & $\begin{array}{l}5: 19 \text {; indicator of } \\
\text { clinic success }\end{array}$ & Variable | Indicator | Clinician \& staff \\
\hline $\begin{array}{l}\text { C/S willingness to meet with } \\
\text { PERC }\end{array}$ & $\begin{array}{l}5: 19 \text {; indicator of } \\
\text { clinic success }\end{array}$ & Variable | Indicator | Clinician \& staff \\
\hline CCO incentive metric & $5: 47 ; 5: 46$ & $\begin{array}{l}\text { Link | Context: external health system | } \\
\text { Variable | Count:2 }\end{array}$ \\
\hline $\begin{array}{l}\text { Clinic ability to have difficult } \\
\text { conversations }\end{array}$ & $5: 8$ & Link | Clinician \& staff \\
\hline $\begin{array}{l}\text { Clinic ability to participate in } \\
\text { project }\end{array}$ & $5: 18$ & Link | Context: local \\
\hline Clinic bandwidth & $5: 7 ; 5: 18: 5: 15$ & Link | Context: local | Count:3 \\
\hline Clinic champion leave & $5: 6$ & Link | Implementation \& intervention \\
\hline $\begin{array}{l}\text { Clinic champion pull weight for QI } \\
\text { project }\end{array}$ & $5: 6$ & $\begin{array}{l}\text { Link | Implementation \& intervention | } \\
\text { Implied }\end{array}$ \\
\hline
\end{tabular}




\begin{tabular}{|c|c|c|}
\hline Clinic champions & $5: 7$ & Link | Implementation \& intervention \\
\hline Clinic conflict resolution capacity & $5: 8$ & Link | Clinician \& staff \\
\hline $\begin{array}{l}\text { Clinic likelihood of signing up for } \\
\text { ANTECEDENT }\end{array}$ & $5: 4$ & Link | Context: local | Implied \\
\hline $\begin{array}{l}\text { Clinic likelihood of signing up for } \\
\text { ORPRN projects }\end{array}$ & $5: 19$ & Link | Context: local \\
\hline Clinic performance & $5: 6$ & Link | Implementation \& intervention \\
\hline Clinic positive team dynamic & $5: 8$ & Link | Context: local \\
\hline Clinic QI decision-making power & $5: 15$ & Link | Context: external health system \\
\hline Clinic size & $5: 6 ; 5: 15 ; 5: 17$ & Link | Context: local | Count:3 \\
\hline Clinic staffing issues & $5: 15$ & Link | Context: local \\
\hline Clinic sustainability plan & $5: 9$ & Variable | Implementation \& intervention \\
\hline Clinic-ORPRN relationship & $5: 10 ; 5: 19 ; 5: 18$ & Link | Context: local | Count:3 \\
\hline Clinics not prioritizing QI & $5: 15$ & Link | Context: local \\
\hline Clinics protective of $\mathrm{C} / \mathrm{S}$ time & $5: 15$ & Link| Context: local \\
\hline $\begin{array}{l}\text { Democratic decision making } \\
\text { structure }\end{array}$ & $5: 6$ & Link | Context: local \\
\hline Effective SBIRT workflow & $5: 9$ & Link | Implementation \& intervention \\
\hline $\begin{array}{l}\text { External organizations trying to } \\
\text { influence clinical practice }\end{array}$ & $5: 4$ & Link | Context: external health system \\
\hline Feedback and evaluation system & $5: 9$ & Variable | Implementation \& intervention \\
\hline Health system affiliation & $5: 15 ; 5: 17$ & $\begin{array}{l}\text { Link | Context: external health system | } \\
\text { Count:2 }\end{array}$ \\
\hline $\begin{array}{l}\text { Likelihood clinic will sign up for QI } \\
\text { projects }\end{array}$ & $5: 15$ & Link| Context: local \\
\hline $\begin{array}{l}\text { Meeting ANTECEDENT grant } \\
\text { requirements }\end{array}$ & $5: 46$ & Link | Implementation \& intervention \\
\hline $\begin{array}{l}\text { Ongoing } \mathrm{C} / \mathrm{S} \text { training and } \\
\text { onboarding }\end{array}$ & $5: 9$ & Link | Implementation \& intervention \\
\hline Patient make positive change & $5: 9$ & Loop | Implementation \& intervention \\
\hline Patient UAU & $5: 9$ & Loop | Implementation \& intervention \\
\hline $\begin{array}{l}\text { PERC ability to offer support to } \\
\text { clinics }\end{array}$ & $5: 8$ & Link | Implementation \& intervention \\
\hline PERC and C/S learning together & $\begin{array}{l}5: 5 ; \text { indicator of } \\
\text { PERC success }\end{array}$ & $\begin{array}{l}\text { Variable | Indicator | Implementation \& } \\
\text { intervention }\end{array}$ \\
\hline $\begin{array}{l}\text { PERC application of knowledge } \\
\text { with clinics }\end{array}$ & $5: 4$ & Loop| Implementation \& intervention \\
\hline PERC collect data from clinics & $5: 46$ & Link | Implementation \& intervention \\
\hline PERC communication skills & $5: 2$ & Link| PERC \\
\hline $\begin{array}{l}\text { PERC connect clinic with subject } \\
\text { matter experts }\end{array}$ & $5: 46$ & Link | Implementation \& intervention \\
\hline $\begin{array}{l}\text { PERC customize message to } \\
\text { patient } \& \text { staff needs }\end{array}$ & $5: 4$ & $\begin{array}{l}\text { Link | Implementation \& intervention } \\
\text { |Implied }\end{array}$ \\
\hline PERC engagement with clinic & $5: 2$ & Link| PERC \\
\hline PERC facilitation skill & $5: 2$ & Variable | PERC \\
\hline
\end{tabular}




\begin{tabular}{|c|c|c|}
\hline PERC knowledge and skill & $5: 4$ & Loop | PERC \\
\hline $\begin{array}{l}\text { PERC motivational interviewing } \\
\text { skill }\end{array}$ & $5: 2$ & Link | PERC \\
\hline PERC orient $\mathrm{C} / \mathrm{S}$ to project & $5: 7$ & Link | Implementation \& intervention \\
\hline $\begin{array}{l}\text { PERC push to get firm no from } \\
\text { clinics }\end{array}$ & $5: 18$ & Link | Implementation \& intervention \\
\hline PERC regional affiliation & $5: 17$ & Link | PERC \\
\hline $\begin{array}{l}\text { PERC support clinic in } \\
\text { implementing project }\end{array}$ & $5: 46$ & Link | Implementation \& intervention \\
\hline PERC training & $5: 4$ & Loop | PERC \\
\hline PERC-C/S relationship & $\begin{array}{l}5: 5 ; 5: 2 \text {; indicator } \\
\text { of PERC success }\end{array}$ & $\begin{array}{l}\text { Variable | Loop | Indicator | PERC | } \\
\text { Clinician \& staff }\end{array}$ \\
\hline Prior ORPRN involvement & $5: 10$ & Link | Context: local \\
\hline SBIRT outcomes & $\begin{array}{l}5: 5 \text {; indicator of } \\
\text { PERC success }\end{array}$ & $\begin{array}{l}\text { Variable | Indicator | Implementation \& } \\
\text { intervention }\end{array}$ \\
\hline Staff filling multiple roles & $5: 15$ & Link | Context: local \\
\hline $\begin{array}{l}\text { Subject matter experts on } \\
\text { ANTECEDENT team }\end{array}$ & $5: 8$ & Link | Implementation \& intervention \\
\hline $\begin{array}{l}\text { Successful change in long-term } \\
\text { SBIRT implementation }\end{array}$ & $\begin{array}{l}5: 9 ; 5: 47: 5: 2 ; 5: 8 \\
5: 46\end{array}$ & $\begin{array}{l}\text { Loop | Implementation \& intervention | } \\
\text { Link | Count:5 }\end{array}$ \\
\hline Time constraints & $5: 7$ & Link | Context: local \\
\hline Trust between PERC and C/S & $5: 5$ & Variable | PERC | Clinician \& staff \\
\hline
\end{tabular}


Causal links and properties

\begin{tabular}{|c|c|c|c|c|}
\hline From & To & $\begin{array}{l}\text { Typ } \\
\text { e }\end{array}$ & $\begin{array}{l}\text { Descr } \\
\text { iption }\end{array}$ & Tags \\
\hline $\begin{array}{l}\text { PERC communication } \\
\text { skills }\end{array}$ & $\begin{array}{l}\text { PERC engagement with } \\
\text { clinic }\end{array}$ & + & $5: 2$ & Link| PERC \\
\hline $\begin{array}{l}\text { PERC engagement with } \\
\text { clinic }\end{array}$ & PERC-C/S relationship & + & $5: 2$ & Link| PERC \\
\hline $\begin{array}{l}\text { PERC motivational } \\
\text { interviewing skill }\end{array}$ & $\begin{array}{l}\text { Successful change in SBIRT } \\
\text { implementation }\end{array}$ & + & $5: 2$ & Link | PERC \\
\hline $\mathrm{C} / \mathrm{S}$ resistance to change & $\begin{array}{l}\text { Successful change in SBIRT } \\
\text { implementation }\end{array}$ & 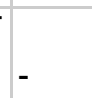 & $5: 2$ & Link | Clinician \& staff \\
\hline $\begin{array}{l}\text { External organizations } \\
\text { trying to influence clinical } \\
\text { practice }\end{array}$ & $\begin{array}{l}\text { Clinic likelihood of signing } \\
\text { up for ANTECEDENT }\end{array}$ & - & $5: 4$ & $\begin{array}{l}\text { Link | Context: external } \\
\text { health system }\end{array}$ \\
\hline $\begin{array}{l}\text { External organizations } \\
\text { trying to influence clinical } \\
\text { practice }\end{array}$ & $\begin{array}{l}\text { PERC customize message } \\
\text { to patient and staff needs }\end{array}$ & + & $5: 4$ & $\begin{array}{l}\text { Link | Context: external } \\
\text { health system }\end{array}$ \\
\hline $\begin{array}{l}\text { PERC customize message } \\
\text { to patient and staff needs }\end{array}$ & $\begin{array}{l}\text { Clinic likelihood of signing } \\
\text { up for ANTECEDENT }\end{array}$ & + & $5: 4$ & Link | PERC \\
\hline PERC training & PERC knowledge and skill & + & $5: 4$ & Loop | PERC \\
\hline PERC knowledge and skill & $\begin{array}{l}\text { PERC application of } \\
\text { knowledge with clinics }\end{array}$ & + & $5: 4$ & $\begin{array}{l}\text { Loop| Implementation \& } \\
\text { intervention }\end{array}$ \\
\hline $\begin{array}{l}\text { PERC application of } \\
\text { knowledge with clinics }\end{array}$ & PERC knowledge and skill & + & $5: 4$ & Loop | PERC \\
\hline C/S buy-in & Clinic performance & + & $5: 6$ & Link | Clinician \& staff \\
\hline Clinic champion leave & $\begin{array}{l}\text { Clinic champion pull weight } \\
\text { for QI project }\end{array}$ & - & $5: 6$ & $\begin{array}{l}\text { Link | Implementation \& } \\
\text { intervention }\end{array}$ \\
\hline $\begin{array}{l}\text { Clinic champion pull weight } \\
\text { for QI project }\end{array}$ & Clinic performance & + & $5: 6$ & $\begin{array}{l}\text { Link | Implementation \& } \\
\text { intervention }\end{array}$ \\
\hline Clinic size & $\begin{array}{l}\text { Democratic decision making } \\
\text { structure }\end{array}$ & + & $5: 6$ & Link | Context: local \\
\hline $\begin{array}{l}\text { C/S knowledge about } \\
\text { project }\end{array}$ & C/S buy-in & + & $5: 7$ & Link | Clinician \& staff \\
\hline $\begin{array}{l}\text { C/S knowledge about } \\
\text { project }\end{array}$ & $\begin{array}{l}\text { C/S understanding what to } \\
\text { expect in project }\end{array}$ & + & $5: 7$ & Link | Clinician \& staff \\
\hline $\begin{array}{l}\text { C/S understanding what to } \\
\text { expect in project }\end{array}$ & C/S buy-in & + & $5: 7$ & Link | Clinician \& staff \\
\hline PERC orient $\mathrm{C} / \mathrm{S}$ to project & C/S buy-in & + & $5: 7$ & $\begin{array}{l}\text { Link | Implementation \& } \\
\text { intervention }\end{array}$ \\
\hline Time constraints & Clinic bandwidth & - & $5: 7$ & Link | Context: local \\
\hline Clinic bandwidth & PERC orient $\mathrm{C} / \mathrm{S}$ to project & - & $5: 7$ & $\begin{array}{l}\text { Link | Implementation \& } \\
\text { intervention }\end{array}$ \\
\hline Clinic champions & C/S buy-in & + & $5: 7$ & $\begin{array}{l}\text { Link | Implementation \& } \\
\text { intervention }\end{array}$ \\
\hline $\mathrm{C} / \mathrm{S}$ trust within team & Clinic positive team dynamic & + & $5: 8$ & Link | Context: local \\
\hline Clinic positive team & Successful change in SBIRT & + & $5: 8$ & Link | Context: local \\
\hline
\end{tabular}




\begin{tabular}{|c|c|c|c|c|}
\hline dynamic & implementation & & & \\
\hline $\begin{array}{l}\mathrm{C} / \mathrm{S} \text { communication within } \\
\text { team }\end{array}$ & Clinic positive team dynamic & + & $5: 8$ & Link | Context: local \\
\hline $\begin{array}{l}\text { Clinic positive team } \\
\text { dynamic }\end{array}$ & $\begin{array}{l}\text { C/S ability to work together } \\
\text { for ANTECEDENT }\end{array}$ & + & $5: 8$ & Link | Context: local \\
\hline $\mathrm{C} / \mathrm{S}$ ego & $\mathrm{C} / \mathrm{S}$ resistance to change & + & $5: 8$ & Link | Clinician \& staff \\
\hline $\begin{array}{l}\text { Clinic conflict resolution } \\
\text { capacity }\end{array}$ & Clinic positive team dynamic & + & $5: 8$ & Link | Clinician \& staff \\
\hline $\begin{array}{l}\text { Clinic positive team } \\
\text { dynamic }\end{array}$ & $\begin{array}{l}\text { Clinic ability to have difficult } \\
\text { conversations }\end{array}$ & + & $5: 8$ & Link | Clinician \& staff \\
\hline $\begin{array}{l}\text { Subject matter experts on } \\
\text { ANTECEDENT team }\end{array}$ & $\begin{array}{l}\text { PERC ability to offer support } \\
\text { to clinics }\end{array}$ & + & $5: 8$ & $\begin{array}{l}\text { Link | Implementation \& } \\
\text { intervention }\end{array}$ \\
\hline $\begin{array}{l}\text { PERC ability to offer } \\
\text { support to clinics }\end{array}$ & Clinic positive team dynamic & + & $5: 8$ & $\begin{array}{l}\text { Link | Implementation \& } \\
\text { intervention }\end{array}$ \\
\hline $\begin{array}{l}\text { C/S see impact of project } \\
\text { on patients }\end{array}$ & C/S buy-in & + & $5: 9$ & $\begin{array}{l}\text { Loop | Implementation \& } \\
\text { intervention }\end{array}$ \\
\hline $\mathrm{C} / \mathrm{S}$ buy-in & $\begin{array}{l}\text { Successful change in long- } \\
\text { term SBIRT implementation }\end{array}$ & + & $5: 9$ & $\begin{array}{l}\text { Loop | Implementation \& } \\
\text { intervention }\end{array}$ \\
\hline $\begin{array}{l}\text { Successful change in long- } \\
\text { term SBIRT } \\
\text { implementation }\end{array}$ & BI with patients & + & $5: 9$ & $\begin{array}{l}\text { Loop | Implementation \& } \\
\text { intervention }\end{array}$ \\
\hline BI with patients & $\begin{array}{l}\text { Patient make positive } \\
\text { change }\end{array}$ & + & $5: 9$ & $\begin{array}{l}\text { Loop | Implementation \& } \\
\text { intervention }\end{array}$ \\
\hline $\begin{array}{l}\text { Patient make positive } \\
\text { change }\end{array}$ & $\begin{array}{l}\mathrm{C} / \mathrm{S} \text { see impact of project on } \\
\text { patients }\end{array}$ & + & $5: 9$ & $\begin{array}{l}\text { Loop | Implementation \& } \\
\text { intervention }\end{array}$ \\
\hline $\begin{array}{l}\text { Patient make positive } \\
\text { change }\end{array}$ & Patient UAU & - & $5: 9$ & $\begin{array}{l}\text { Loop | Implementation \& } \\
\text { intervention }\end{array}$ \\
\hline $\begin{array}{l}\text { Ongoing } \mathrm{C} / \mathrm{S} \text { training and } \\
\text { onboarding }\end{array}$ & Effective SBIRT workflow & + & $5: 9$ & $\begin{array}{l}\text { Link | Implementation \& } \\
\text { intervention }\end{array}$ \\
\hline Prior ORPRN involvement & Clinic-ORPRN relationship & + & $5: 10$ & Link | Context: local \\
\hline Clinic-ORPRN relationship & $\begin{array}{l}\text { Ability to get meetings with } \\
\text { clinics }\end{array}$ & + & $5: 10$ & Link | Context: local \\
\hline Clinic staffing issues & Clinic bandwidth & - & $5: 15$ & Link | Context: local \\
\hline Clinic size & Staff filling multiple roles & - & $5: 15$ & Link | Context: local \\
\hline Staff filling multiple roles & Clinic bandwidth & - & $5: 15$ & Link | Context: local \\
\hline Health system affiliation & $\begin{array}{l}\text { Clinic QI decsion-making } \\
\text { power }\end{array}$ & - & $5: 15$ & $\begin{array}{l}\text { Link / Context: external } \\
\text { health system }\end{array}$ \\
\hline $\begin{array}{l}\text { Clinics protective of } \mathrm{C} / \mathrm{S} \\
\text { time }\end{array}$ & $\begin{array}{l}\text { Likelihood clinic will sign up } \\
\text { for QI projects }\end{array}$ & - & $5: 15$ & Link| Context: local \\
\hline Clinics not prioritizing QI & $\begin{array}{l}\text { Clinics protective of } \mathrm{C} / \mathrm{S} \\
\text { time }\end{array}$ & + & $5: 15$ & Link | Context: local \\
\hline Clinic size & $\begin{array}{l}\text { C/S perception of } \\
\text { improvement need }\end{array}$ & - & $5: 17$ & Link | Context: local \\
\hline Health system affiliation & $\begin{array}{l}\text { C/S perception of } \\
\text { improvement need }\end{array}$ & - & $5: 17$ & Link | Context: local \\
\hline
\end{tabular}




\begin{tabular}{|c|c|c|c|c|}
\hline PERC regional affiliation & $\mathrm{C} / \mathrm{S}$ receptiveness to $\mathrm{PERC}$ & + & $5: 17$ & Link | PERC \\
\hline $\begin{array}{l}\mathrm{C} / \mathrm{S} \text { reluctance to meddling } \\
\text { from outsiders from } \\
\text { Portland }\end{array}$ & $\mathrm{C} / \mathrm{S}$ receptiveness to $\mathrm{PERC}$ & - & $5: 17$ & Link | Clinician \& staff \\
\hline Clinic bandwidth & $\begin{array}{l}\text { Clinic ability to participate in } \\
\text { project }\end{array}$ & + & $5: 18$ & Link | Context: local \\
\hline $\begin{array}{l}\text { PERC push to get firm no } \\
\text { from clinics }\end{array}$ & Clinic-ORPRN relationship & - & $5: 18$ & $\begin{array}{l}\text { Link | Implementation \& } \\
\text { intervention }\end{array}$ \\
\hline $\begin{array}{l}\mathrm{C} / \mathrm{S} \text { perception of project } \\
\text { value }\end{array}$ & Clinic-ORPRN relationship & + & $5: 19$ & Link | Context: local \\
\hline Clinic-ORPRN relationship & $\begin{array}{l}\text { Clinic likelihood of signing } \\
\text { up for ORPRN projects }\end{array}$ & + & $5: 19$ & Link | Context: local \\
\hline $\begin{array}{l}\text { PERC connect clinic with } \\
\text { subject matter experts }\end{array}$ & $\begin{array}{l}\text { Successful change in SBIRT } \\
\text { implementation }\end{array}$ & + & $5: 46$ & $\begin{array}{l}\text { Link | Implementation \& } \\
\text { intervention }\end{array}$ \\
\hline $\begin{array}{l}\text { PERC support clinic in } \\
\text { implementing project }\end{array}$ & $\begin{array}{l}\text { Successful change in SBIRT } \\
\text { implementation }\end{array}$ & + & $5: 46$ & $\begin{array}{l}\text { Link | Implementation \& } \\
\text { intervention }\end{array}$ \\
\hline $\begin{array}{l}\text { PERC collect data from } \\
\text { clinics }\end{array}$ & $\begin{array}{l}\text { Meeting ANTECEDENT } \\
\text { grant requirements }\end{array}$ & + & $5: 46$ & $\begin{array}{l}\text { Link | Implementation \& } \\
\text { intervention }\end{array}$ \\
\hline CCO incentive metric & C/S buy-in & + & $5: 47$ & $\begin{array}{l}\text { Link | Context: external } \\
\text { health system }\end{array}$ \\
\hline $\begin{array}{l}\text { Successful change in } \\
\text { SBIRT implementation }\end{array}$ & C/S buy-in & + & $5: 47$ & $\begin{array}{l}\text { Link | Implementation \& } \\
\text { intervention }\end{array}$ \\
\hline
\end{tabular}


Appendix E: Selection of diagrams produced during initial interviews

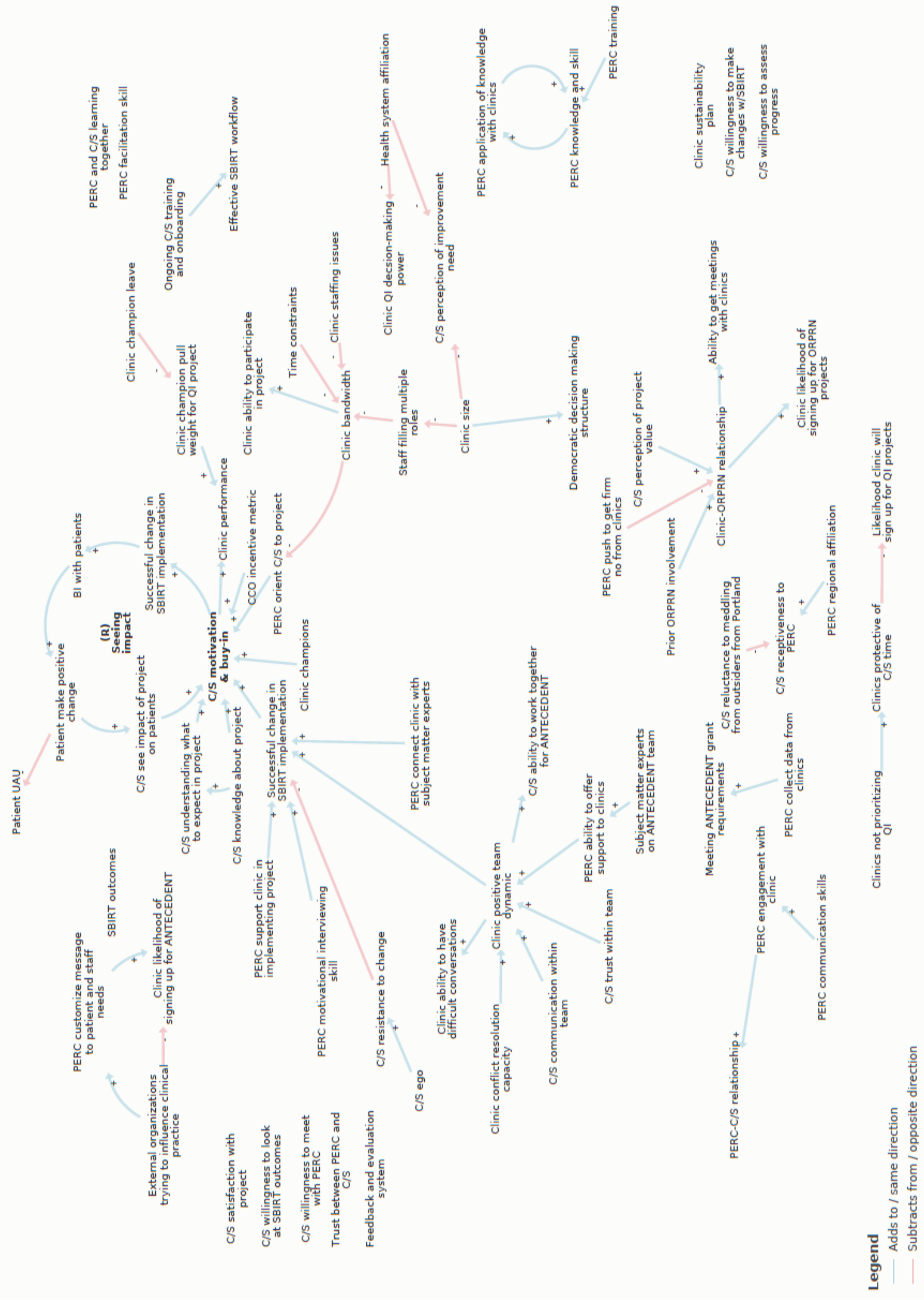




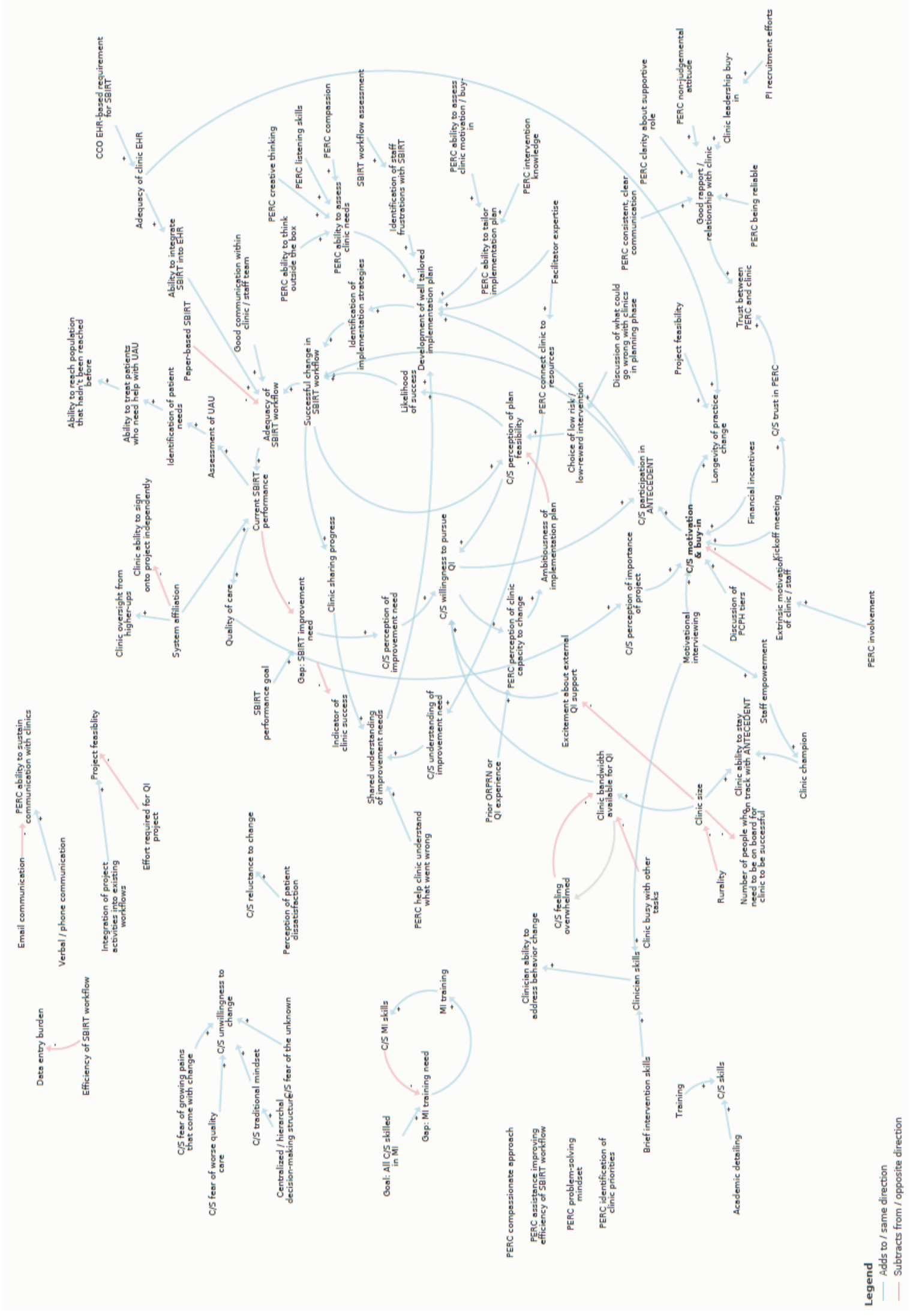




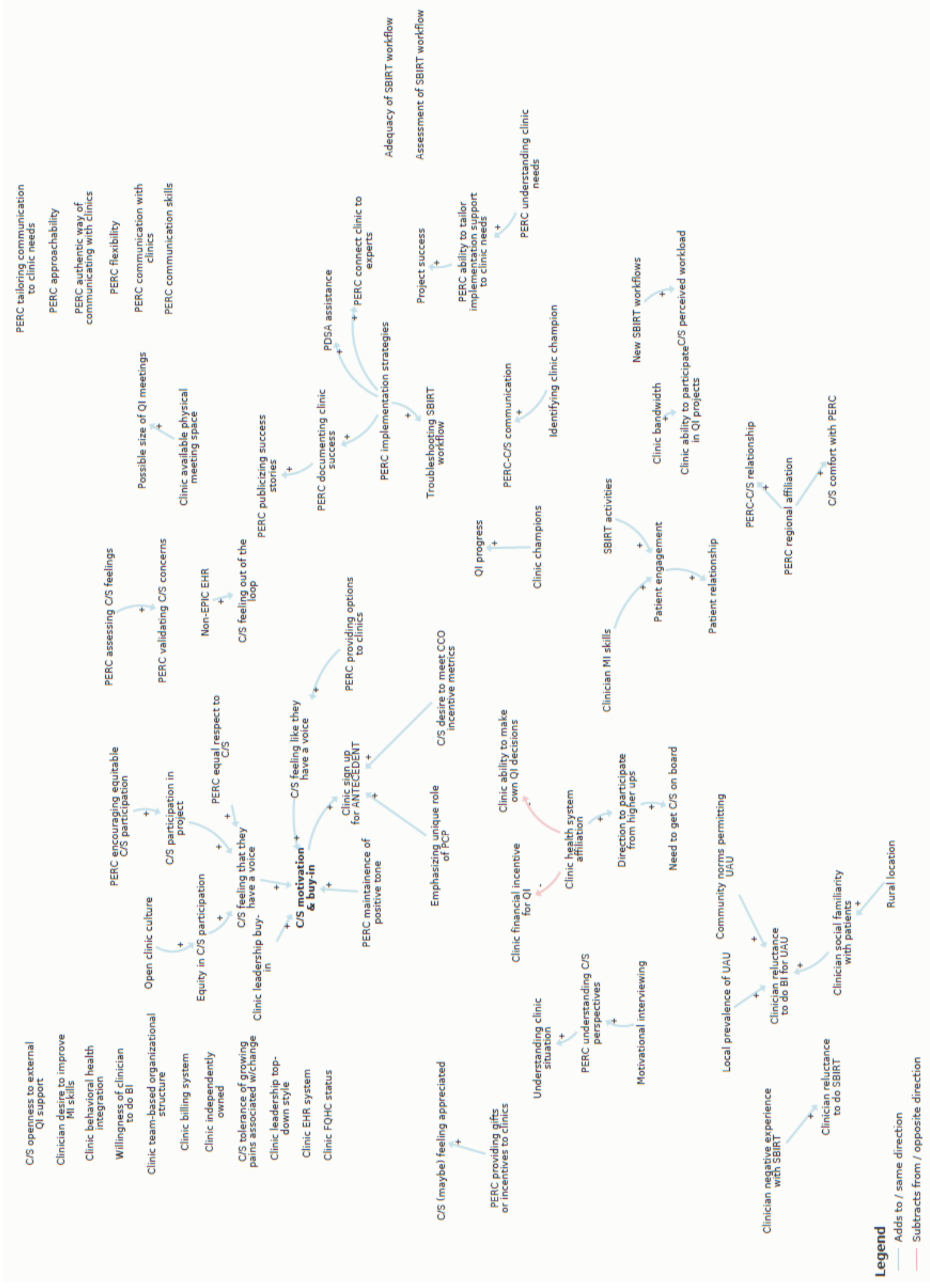

